\title{
Poynting vector, super-Poynting vector, and principal observers in electromagnetism and general relativity
}

\author{
May 31, 2021
}

\author{
Lode Wylleman ${ }^{\diamond} \dagger$, L. Filipe O Costa ${ }^{\star}$ and José Natário* \\ ${ }^{\diamond}$ Faculty of Science and Technology, University of Stavanger, N-4036 Stavanger, Norway \\ ${ }^{\dagger}$ Department of Mathematical Analysis, Ghent University, Galglaan 2, 9000 Gent, Belgium \\ * CAMGSD, Departamento de Matemática, Instituto Superior Técnico, 1049-001 Lisboa, Portugal \\ Email: lode.wylleman@uis.no, lfilipecosta@tecnico.ulisboa.pt, jnatar@math.ist.utl.pt
}

\begin{abstract}
In electromagnetism, the concept of Poynting vector as measured by an observer is well known. A mathematical analogue in general relativity is the super-Poynting vector of the Weyl tensor. Observers for which the (super-)Poynting vector vanishes are called principal. When, at a given point, the electromagnetic field is non-null, or the gravitational field is of Weyl-Petrov type I or D, principal observers instantaneously passing through that point always exist. We survey characterizations of such observers and study their relation to arbitrary observers. In the non-null electromagnetic case it is known that, given any observer, there is a principal observer which moves relative to the first in the direction of his Poynting vector. Replacing Poynting by super-Poynting yields a possible gravitational analogue; we show that this analogy indeed holds for any observer when the Petrov type is D, but only for a one-dimensional variety of observers when the Petrov type is I. We provide algorithms to obtain the principal observers directly from the electric and magnetic fields (in the electromagnetic case) or electric and magnetic parts of the Weyl tensor (in the gravitational case) relative to an arbitrary observer. It is found that in Petrov type D doubly aligned non-null Einstein-Maxwell fields (which include all classical charged black hole solutions) the Poynting and super-Poynting vectors are aligned, at each point and for each observer, and the principal observers coincide. Our results are illustrated in simple examples.
\end{abstract}

\section{Contents}

1 Introduction

2 Executive summary

3 Preliminaries 6

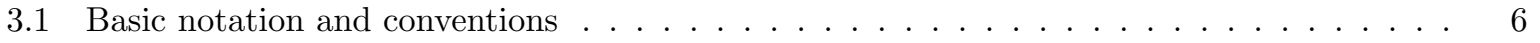

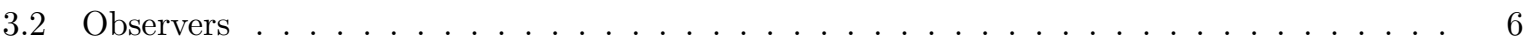

3.3 Self-dual bivectors $\ldots \ldots \ldots \ldots \ldots \ldots \ldots \ldots \ldots \ldots$

4 The electromagnetic case 8

4.1 EM principal observers . . . . . . . . . . . . . . . . 8

4.2 Non-null fields: general vs principal observers . . . . . . . . . . . . . . . . . . 10 
5 The gravitational case 14

5.1 Weyl principal observers . . . . . . . . . . . . . . . . . . . . . . . . 14

5.2 Petrov type D: general vs. principal observers . . . . . . . . . . . . . . . . 18

5.2 .1 Doubly aligned non-null Einstein-Maxwell fields . . . . . . . . . . . . . . . 21

5.3 Petrov type I: general vs. principal observers $\ldots \ldots \ldots \ldots \ldots \ldots$

6 Examples 22

6.1 Electromagnetic field of a spinning charge . . . . . . . . . . . . . . . . . 29

6.2 Kerr-Newman spacetimes . . . . . . . . . . . . . . . . . . . . 30

6.3 Kasner spacetimes . . . . . . . . . . . . . . . . . . . . . . . . . 333

7 Summary and discussion 34

A Orthonormal frames in self-dual bivector and tangent space

B Notes on the Weyl and Bel-Robinson tensors 36

B.1 Petrov classification of the Weyl tensor in terms of $Q^{a}{ }_{b} \ldots \ldots \ldots \ldots \ldots \ldots \ldots$

B.2 Bel-Robinson endomorphism and degenerate Petrov type I . . . . . . . . . . . . 37

C Principal observer conditions

\section{Introduction}

In the late 1950's, and in the framework of classical general relativity theory, Bel published a series of influential notes [1, culminating in his $\mathrm{PhD}$ thesis [2]. Important concepts and results appear for the first time in these notes: the refinement of the Petrov type classification of the Weyl tensor 1 the superenergy tensor [3] of the Weyl tensor which later became known as the Bel-Robinson tensor, and new or precise definitions of quantities relative to an observer: the electric and magnetic parts of the Weyl tensor (inspired by Matte's work [4) and the super-energy density and super-Poynting vector.

The motivation for introducing the last two quantities is the following: an old problem in general relativity is that although it is known that the gravitational field and waves carry some sort of energy and momentum (as given e.g. by the Landau-Lifshitz pseudotensor [5]), such quantities cannot be defined as tensors and have no local meaning since, due to the equivalence principle, they can always be made to locally vanish. The Bel-Robinson tensor (and its associated super-energy scalar and super-Poynting vector) arose in a quest to devise quantities formally analogous to the electromagnetic stress-energy tensor, in terms of which one could define a scalar and a vector analogous to the electromagnetic energy density and Poynting vector. The price to pay is that these are quantities with strange dimensions and whose physical meaning is no longer clear [3, 6, 7]. Nevertheless, some suggestive connections between super-energy and the quasi-local notions of gravitational field energy density (e.g. Landau-Lifshitz's) can be found in the literature $[3,8,9,10$. It is moreover believed that gravitational radiation is accompanied by a super-Poynting vector [11, 12, 13, 14, 15, 16; ; in the linear regime, this is clearly suggested by Matte's equations [17, and, recently, a relationship with the quasi-local wave energy flux has been put forth [16]; in the exact theory, it has also recently been shown [14 that a variant of it yields a characterization of the presence of gravitational radiation equivalent to the classical one, given by the News tensor. This fosters the interest in studying the observers relative to which the super-Poynting vector vanishes, and led to the proposal, inspired by the electromagnetic analogy, of the non-existence of such observers at a given point as a criterion for "intrinsic radiation" 12. Such research line started with the seminal work by Pirani [11, who studied the eigenbivectors of the Weyl tensor (considered as an endomorphism of self-dual bivector space, as in the original Petrov classification), calling the intersections of the corresponding 2-planes 'principal vectors'. Observers with a timelike principal vector as their 4-velocity at a point

\footnotetext{
${ }^{1}$ Strictly speaking, most of the original results apply to the Riemann tensor of a pure vacuum or Einstein spacetime, but they can be and have been generalized to the Weyl tensor in the presence of matter, and are here stated as such.
} 
were dubbed to be instantaneously 'following the gravitational field', because in the electromagnetic (henceforth abbreviated to EM) analogue they correspond to observers measuring a vanishing Poynting vector; we shall call them principal observers here. Pirani proved that when the Petrov type is 'diagonal' at the point, i.e., algebraically general type I or type $\mathrm{D}$ or trivial type $\mathrm{O}$ (corresponding to a vanishing Weyl tensor) then, and only then, principal observers exist. In 12] Bel pointed out the correct difference between the non-trivial Petrov types I and D in this respect, showing that in the Petrov type I case only one observer is principal, while in the Petrov type $\mathrm{D}$ case the principal observers are precisely those observers lying in the 2-plane spanned by the repeated principal null directions of the Weyl tensor. Bel moreover identified the principal observers as those relative to which the super-Poynting vector vanishes, and demonstrated that this precisely happens when the electric and magnetic parts of the Weyl tensor (considered as endomorphisms of tangent space) can be simultaneously diagonalized in a real orthonormal (eigen)frame.

It is worth emphasizing that a generic spacetime is of Weyl-Petrov type I at each point and thus allows for a unique congruence of Weyl principal observers. On taking an orthonormal tetrad formalism where the timelike tetrad vector is the principal one, the classification and determination of all Petrov type I spacetimes with given additional properties becomes feasible; see e.g. [18, for a class of algebraically general rotating dust spacetimes. Many examples of Petrov type D spacetimes exist [19]: the Petrov type D vacuum spacetimes and doubly aligned non-null Einstein-Maxwell fields, which have been fully classified (with or without cosmological constant) [20, 21] and contain well-known black hole solutions such as the Schwarzschild, Reissner-Nordström, Kerr, Kerr-Newman, Kerr-NUT-(A)dS, (charged or spinning) $C$ and Plebański-Demiański spacetimes; all spherically symmetric spacetimes like the Lemaitre-Tolman-Bondi dust models, and the asymmetric Szekeres dust model, to name a few; some notable congruences of observers in these solution families (e.g. the static observers in Schwarzschild, or the Carter observers in the Kerr and Kerr-Newman cases) are actually Weyl principal.

In the present paper we will focus on the pointwise relation of general observers to principal ones. Much of the aforementioned work in the gravitational case has been inspired by the EM setting; here observers with respect to which the Poynting vector vanishes will also be called principal. For a non-zero Faraday tensor principal observers exist precisely when the EM field is non-null, and are those observers lying in the 2-plane spanned by the principal null directions. It is known that given any observer $u^{a}$, there is a (unique) principal observer $u_{\mathrm{em}}^{a}$ which travels instantaneously relative to $u^{a}$ in the direction of the Poynting vector $p^{a}$ measured by $u^{a}$, with speed $\tanh (\psi)$ implicitly given by the Wheeler equation

$$
\tanh (2 \psi)=\sqrt{p^{a} p_{a}} / \rho_{\mathrm{em}}
$$

where $\rho_{\mathrm{em}}$ is the EM energy density as measured by $u^{a}$; this will be referred to as the 'EM Wheeler result' ${ }^{2}$ In his contribution 24] Wheeler emphasized the "miracle" of this result. He also scrutinized whether an analogous result could hold in the gravitational case with diagonal Petrov type - on replacing Poynting by super-Poynting vector and with a surrogate of (1), which will be referred to as the 'gravitational Wheeler analogue' - but came to negative conclusions. In the present paper we will clarify the EM Wheeler result, prove that the gravitational Wheeler analogue does hold when the Weyl tensor is of Petrov type D at a spacetime point, and find that it is invalid for general but valid for some observers when the Petrov type is I, thereby refining Wheeler's negative conclusions in this case.

In 24] Wheeler also studied the possibility of obtaining analytically the boost(s) that annihilate the super-Poynting vector [i.e., boost(s) relating a general observer $u^{a}$ to the principal one(s)] directly from the electric and magnetic parts of the Weyl tensor as measured by an arbitrary observer, but deemed this impossible. In this paper, however, we show that this can be done, and design simple algorithms for this purpose.

Outline of the paper. Sec. 2 provides an executive summary, where our main results are briefly outlined. Sec. 3 fixes the notation and conventions and provides a technical background.

2 The result is already implicit in Synge's standard work 22. To our knowledge, it makes its first appearance in the caption of Figure 1 of [23], albeit with an error in the description. The result is mentioned as a problem in section 25 of 5 ] and proved in 24], producing the correct formula [1]; see also Ex. 20.6 in [25], and [26]. 
Sec. 4 treats the EM case. First we survey characterizations, existence and locus of EM principal observers. Then we derive a version of the EM Wheeler result (theorem 4.3 which explicates its geometric nature and provides a simple, explicit formula for the velocity of $u_{\mathrm{em}}^{a}$ relative to $u^{a}$. We also provide an algorithm that computes all principal observers from the electric and magnetic fields as measured by an arbitrary observer.

Sec. 5 deals with the gravitational case. Again we first survey characterizations, existence and loci of Weyl principal observers. Next, we derive our main result, namely the gravitational Wheeler analogue for Petrov type D (theorem 5.5), and point out how to deduce all principal observers from the electric and magnetic parts of the Weyl tensor as measured by an arbitrary observer. As an application we find that in Petrov type D doubly aligned non-null Einstein-Maxwell fields the Poynting and super-Poynting vectors are aligned, at each point and for each observer, and the principal observers coincide. Finally, the Petrov type I case is studied; we show that the observers for which the gravitational Wheeler analogue does hold form a one-dimensional variety (i.e., a finite union of curves and points), which we pin down in the 'degenerate' Petrov type I subcases in the sense of [27, and present an algorithm that derives the unique principal observer directly from the electric and magnetic parts of the Weyl tensor measured by an arbitrary observer.

Sec. 6illustrates our results in simple examples: the non-null EM field of a spinning charge, the Petrov type D Kerr-Newman spacetimes, and the Petrov type I Kasner spacetimes.

Sec. 7 7 summarizes our results and comments on the physical significance of the super-Poynting vector.

Supporting or additional material is put in three appendices: a geometric proof of the bijection between oriented orthonormal frames in self-dual bivector and tangent space, notes on the Petrov classification of the Weyl tensor and 'degenerate' Petrov type I, and a simple proof of the minimal super-energy density characterization of Weyl principal observers.

\section{Executive summary}

In electromagnetism, for a non-null Faraday tensor (i.e., in an electromagnetic field which is not pure radiation), there are observers for whom the Poynting vector vanishes (and whose measured electromagnetic energy density is minimum). These are called EM principal observers. Given the electric and magnetic fields $E^{a}$ and $B^{a}$ as measured by some arbitrary observer (of 4 -velocity) $u^{a}$, the principal observers $u^{\prime a}$ are those that are boosted (with respect to $u^{a}$ ) with a relative velocity $v\left(u^{\prime}, u\right)^{a}=v_{\| p}^{a}+v_{\| \mathfrak{e}}^{a}$ having a fixed component $\left(v_{\| p}^{a}\right)$ along the Poynting vector $p^{a}=\epsilon_{b c d}^{a} E^{b} B^{c} u^{d}$,

$$
\begin{aligned}
& v_{\| p}^{a}=\frac{1}{\rho_{\mathrm{em}}+\left|I_{F}\right| / 8 \pi} p^{a} ; \\
& \rho_{\mathrm{em}} \equiv T_{a b} u^{a} u^{b}=\frac{1}{8 \pi}\left(E^{a} E_{a}+B^{a} B_{a}\right), \\
& I_{F} \equiv E^{a} E_{a}-B^{a} B_{a}-2 i E^{a} B_{a}
\end{aligned}
$$

(Electromagnetism, non-null $F_{a b}$ )

(where $T_{a b}$ is the EM energy-momentum tensor), and an arbitrary component $\left(v_{\| \mathfrak{e}}^{a}\right)$ along

$$
\mathfrak{e}^{a}=\Re\left(\frac{f^{a}}{\sqrt{I_{F}}}\right), \quad \quad f^{a} \equiv E^{a}-i B^{a} .
$$

The EM principal observers form then, at each point, an infinite class, consisting of all unit vectors belonging to the distinguished timelike plane $\Sigma_{F}$ spanned by the two principal null directions of the Faraday tensor, called the timelike EM principal plane. Given $u^{a}$, a canonically associated EM principal observer can always be found by boosting in the direction of $p^{a}$ (with relative velocity $v_{\| p}^{a}$ ); geometrically, this is the principal observer obtained by projecting $u^{a}$ onto $\Sigma_{F}$, and the vector $\mathfrak{e}^{a}$ above lies along the aligned electric and magnetic fields measured by this principal observer. 
Here we treat the analogous gravitational problem, first posed by Wheeler: given the electric and magnetic parts $E_{a b}$ and $H_{a b}$ of a non-zero Weyl tensor measured by an arbitrary observer $u^{a}$, find the observers for which the so-called super-Poynting vector vanishes. Such observers are dubbed Weyl principal and exist only for Petrov type I and D spacetimes.

The type D case exhibits a strong analogy with the EM counterpart: the principal observers $u^{\prime a}$ are those that are boosted (with respect to $u^{a}$ ) with a relative velocity $v\left(u^{\prime}, u\right)^{a}=v_{\| \mathcal{P}}^{a}+v_{\| \mathfrak{e}}^{a}$ having a fixed component $\left(v_{\| \mathcal{P}}^{a}\right)$ along the super-Poynting vector $\mathcal{P}^{a}=\frac{1}{2} \epsilon_{b c d}^{a} E^{b}{ }_{e} H^{e c} u^{d}$,

$$
\begin{array}{ll}
v_{\| \mathcal{P}}^{a}=\frac{8}{3|I| \zeta_{\mathrm{g}}\left(\zeta_{\mathrm{g}}+1\right)} \mathcal{P}^{a} ; & \\
\zeta_{\mathrm{g}} \equiv \sqrt{\frac{1}{3}\left(\frac{8 \rho_{\mathrm{g}}}{|I|}+1\right)}, & \text { (Gravity, } \\
\rho_{\mathrm{g}} \equiv T_{a b c d} u^{a} u^{b} u^{c} u^{d}=\frac{1}{4}\left(E^{a b} E_{a b}+H^{a b} H_{a b}\right), & \text { Petrov type D) } \\
I=E^{a b} E_{a b}-H^{a b} H_{a b}-2 i E^{a b} H_{a b} &
\end{array}
$$

(where $T_{a b c d}$ is the Bel-Robinson tensor), and an arbitrary component $\left(v_{\| \mathfrak{e}}^{a}\right)$ along $\mathfrak{e}^{a}$. The vector $\mathfrak{e}^{a}$ is the real part of the (up to sign unique) unit eigenvector associated to the simple eigenvalue of the complex tensor $Q^{a}{ }_{b} \equiv E^{a}{ }_{b}-i H^{a}{ }_{b}$; it is given by

$$
\begin{aligned}
& \mathfrak{e}^{a}=\Re\left(\frac{w^{a}}{\sqrt{w^{b} w_{b}}}\right) ; \quad w^{a}=\left(E^{a}{ }_{b}-i H^{a}{ }_{b}+J h^{a}{ }_{b} / I\right) y^{b}, \\
& J=E_{b}^{a} E_{c}^{b} E_{a}^{c}+i H_{b}^{a} H^{b}{ }_{c} H_{a}^{c}-3 i E_{b}^{a}\left(E^{b}{ }_{c}-i H^{b}{ }_{c}\right) H_{a}^{c}, \quad h_{a b} \equiv g_{a b}+u_{a} u_{b},
\end{aligned}
$$

where $y^{a}$ is any real spatial vector (with respect to $u^{a}$ ) which is not an eigenvector associated to the repeated eigenvalue of $Q^{a}{ }_{b}$; i.e. (in the generic case that $u^{a}$ is non-principal) any vector non-proportional to the super-Poynting vector $\mathcal{P}^{\alpha}$. The Weyl principal observers exhibit features analogous to their EM counterparts: i) at each point, infinitely many such observers exist, namely all unit vectors lying in the timelike Weyl principal plane $\Sigma_{C}$, spanned by the two principal null directions of the Weyl tensor; ii) given $u^{a}$, a canonically associated Weyl principal observer can always be found by boosting in the direction of $\mathcal{P}^{a}$ (with relative velocity $v_{\| \mathcal{P}}^{a}$ ), namely the one obtained by projecting $u^{a}$ onto $\Sigma_{C}$; iii) the Weyl principal observers are those measuring minimum super-energy. The analogy extends to the shape of the field of the Poynting/super-Poynting vectors and principal observers in physically analogous settings (namely a spinning charge vs the Kerr spacetime); in Petrov type D doubly aligned non-null Einstein-Maxwell fields (such as the Kerr-Newman spacetime) one has $\Sigma_{F}=\Sigma_{C}$, the EM and Weyl principal observers coincide, and the Poynting and super-Poynting vectors are aligned, at each point.

In the Petrov type I case the situation differs; at each point there is now a unique Weyl principal observer $e_{0}^{a}$, which in general is not obtained by boosting in the direction of the super-Poynting vector. It has relative velocity

$$
\begin{array}{ll}
v^{a} \equiv v\left(e_{0}, u\right)^{a}=-\frac{1}{\gamma^{2}} \sum_{(i j k)} \Im\left(x_{j}^{b}\left(\overline{x_{k}}\right)_{b}\right) x_{i}^{a} ; & \\
\gamma^{2}=\frac{1}{2}\left[\sum_{i=1}^{3} x_{i}^{a}\left(\bar{x}_{i}\right)_{a}+1\right] ; \quad x_{i}^{a}=\frac{\left(R^{i}\right)_{b}^{a} y^{b}}{\sqrt{R_{c d}^{i} y^{c} y^{d}}}, & \text { (Gravity, } \\
\left(R^{i}\right)^{a}{ }_{b}=\frac{Q^{a}{ }_{c} Q^{a}{ }_{c}+\lambda_{i} Q^{a}{ }_{b}+\left(\lambda_{i}^{2}-I / 2\right) h^{a}{ }_{b}}{3 \lambda_{i}^{2}-I / 2} &
\end{array}
$$


where $y^{a}$ is any real spatial vector which is not an eigenvector of $Q^{a}{ }_{b}$ with eigenvalue $\lambda_{i}$. Only for a one-dimensional variety of observers $u^{a}$ one has $v^{a} \propto \mathcal{P}^{a}$; this variety always contains the three curves of observers obtained by arbitrarily boosting the principal observer along one of the spatial directions of the Weyl principal tetrad, but contains other observers as well, depending on the Weyl parameters; we identify these additional observers for types $\mathrm{I}\left(M^{\infty}\right), \mathrm{I}\left(M^{+}\right)$and $\mathrm{I}\left(M^{-}\right)$within the extended Petrov classification by Arianrhod and McIntosh [27.

\section{Preliminaries}

\subsection{Basic notation and conventions}

$\S 1$. We work at a point $p$ of a spacetime $\left(M, g_{a b}\right)$ with metric signature $(-+++)$, and use units where $8 \pi G=c=1$, with $G$ the gravitational constant and $c$ the speed of light. For tensors abstract index notation with small Latin letters $a, b, c, d, e, f$ is used. Abstract indices are lowered and raised by contraction with $g_{a b}$, resp. $g^{a b}\left(g^{a c} g_{c b}=\delta_{b}^{a}\right.$, with $\delta_{b}^{a}$ the identity (1,1)-tensor), and round (square) brackets around indices indicate (anti)symmetrization. $\Lambda^{2}$ stands for the space of real 2-forms $X_{a b}=X_{[a b]}$ at $p$. In $T_{p} M, S^{\perp}$ denotes the $g$-orthogonal complement of a vector $S^{a}$ or set of vectors $S$, and $\left\langle x_{1}^{a}, x_{2}^{a}, \ldots\right\rangle$ the subspace spanned by $x_{1}^{a}, x_{2}^{a}, \ldots$ A tuple $\left(e_{0}^{a}, e_{i}^{a}\right)$ symbolizes a restricted orthonormal tetrad of $T_{p} M$ (i.e., the tetrad is properly oriented and $e_{0}^{a}$ is future-pointing [28]) and Latin letters $i, j, k, l, m, n$ are triad indices taking values from 1 to 3 . In such a tetrad we take the convention $\epsilon_{0123}=-1$ for the Levi-Civita pseudo-tensor $\epsilon_{a b c d}=\epsilon_{[a b c d]}$. When the labels $i, j, k$ appear in one expression, e.g. $\mu_{i}=\lambda_{j}-\lambda_{k}$, then $(i, j, k)$ is a cyclic permutation of $(1,2,3) ; \sum_{(i j k)}$ denotes cyclic summation. For both abstract and triad indices the Einstein summation convention (only) applies to two indices in opposite (upper and lower) positions, unless stated otherwise. We abbreviate e.g. three-dimensional to 3d.

$\S 2 . \quad \mathbb{C} V \equiv \mathbb{C} \otimes V$ denotes the complexification of a real vector space $V$. Complex conjugation is symbolized by a bar, $\Re$ and $\Im$ indicate real and imaginary parts; $|z|=\sqrt{\Re(z)^{2}+\Im(z)^{2}}$ denotes the modulus of $z \in \mathbb{C}$; we will use that the square roots of $z$ are $\pm \sqrt{z}$ with

$$
\sqrt{z} \equiv \alpha+i \beta, \quad \frac{1}{\sqrt{z}}=\frac{1}{|z|}(\alpha-i \beta), \quad \alpha \equiv \sqrt{\frac{|z|+\Re(z)}{2}}, \quad \beta \equiv \operatorname{sgn}(\Im(z)) \sqrt{\frac{|z|-\Re(z)}{2}},
$$

and where $\operatorname{sgn}: \mathbb{R} \rightarrow\{-1,1\}$ is defined by $\operatorname{sgn}(x)=1$ if $x>0$ and $\operatorname{sgn}(x)=-1$ if $x \leq 0$. For endomorphisms $f, g$ of $V$ or $\mathbb{C} V$ we write $f g \equiv f \circ g$ and inductively define $f^{i+1} \equiv f^{i} f ; \operatorname{Ker}(f), \operatorname{Im}(f)$ and $\operatorname{tr}(f)$ respectively denote the kernel, image and trace of $f . T^{a}{ }_{b}$ is seen as an endomorphism of $\mathbb{C} T_{p} M$. The Hodge dual of a bivector $A_{a b} \in \mathbb{C} \Lambda^{2}$ is denoted $\star A_{a b} \equiv \frac{1}{2} \epsilon_{a b c d} A^{c d}$. On $\mathbb{C} T_{p} M$ and $\mathbb{C} \Lambda^{2}$ we consider the respective metrics

$$
g:\left(v^{a}, w^{a}\right) \mapsto g_{a b} v^{a} w^{b}=v^{a} w_{a}, \quad G:\left(A_{a b}, B_{a b}\right) \mapsto-\frac{1}{4} A^{a b} B_{a b} .
$$

A vector $v^{a} \in \mathbb{C} T_{p} M$ with $v^{a} v_{a}=1$ is called unit, and a bivector $A_{a b} \in \mathbb{C} \Lambda^{2}$ with $-\frac{1}{4} A^{a b} A_{a b}=1$ unitary.

\subsection{Observers}

$\S 1$. Let $U^{+}$denote the set of future-pointing, normalized timelike vectors $u^{a}$ at $p\left(u^{a} u_{a}=-1\right.$ and $u^{0}>0$ in a restricted orthonormal tetrad). An observer is identified with a worldline with normalized tangent vector field $u^{a}$ (the observer's 4 -velocity). Since the quantities treated in the present paper depend only on the observer's 4-velocity and position, we shall for short speak about the observer $u^{a}\left(\in U^{+}\right)$at $p$. The tensor $h_{b}^{a}$ defined by

$$
h_{a b} \equiv g_{a b}+u_{a} u_{b}
$$

represents the projector onto the (complexified) instantaneous rest space $(\mathbb{C}) u^{\perp}$ of $u^{a}$ at $p$. A tensor $T^{a \cdots}{ }_{b \ldots}$ that equals $h_{c}^{a} \cdots h_{b}{ }^{d} \cdots T^{c \cdots}{ }_{d \ldots}$ is called spatial (with respect to $u^{a}$ ). A spatial tensor $T_{a b}$ induces an endomorphism $\nu^{a} \mapsto T_{b}^{a} \nu^{b}$ of $\mathbb{C} u^{\perp}$ which we symbolize by $\mathrm{T}$; hence $\mathrm{h}$ is the identity map of $\mathbb{C} u^{\perp}$. 
When $S_{a b}$ and $T_{a b}$ are spatial and symmetric, the vector dual to the commutator ST - TS (with associated $4 \mathrm{~d}$ tensor $\left.[\mathrm{S}, \mathrm{T}]_{b}^{a} \equiv S^{a}{ }_{c} T_{b}^{c}-T^{a}{ }_{c} S_{b}^{c}\right)$ is

$$
[\mathrm{S}, \mathrm{T}]^{a} \equiv \epsilon_{b c d}^{a} u^{d} S^{b}{ }_{e} T^{e c} .
$$

For spatial vectors $x^{a}, y^{a} \in \mathbb{C} u^{\perp}$ we write $[x, y]^{a} \equiv \epsilon^{a} b c d u^{d} x^{b} y^{c}$ for their vector product, xy for the endomorphism $\nu^{a} \mapsto\left(\nu^{b} y_{b}\right) x^{a}$ of $\mathbb{C} u^{\perp}$, and we will use the identity

$$
[\mathrm{xx}, \mathrm{yy}]^{a}=x^{b} y_{b}[x, y]^{a} .
$$

§2. The relative motion of two observers $u_{1}^{a}, u_{2}^{a} \in U^{+}$is described by (see, e.g., [26, 29])

$$
u_{2}^{a}=\cosh (\psi)\left[u_{1}^{a}+v^{a}\right]=\cosh (\psi) u_{1}^{a}+\sinh (\psi) \widehat{v}^{a} .
$$

Here $v^{a} \equiv v\left(u_{2}, u_{1}\right)^{a} \in u_{1}^{\perp}$ is the velocity of $u_{2}^{a}$ relative to $u_{1}^{a}$, and $\psi \equiv \psi\left(u_{2}, u_{1}\right)=\psi\left(u_{1}, u_{2}\right) \geq 0$ and $\cosh (\psi)=-u_{2}^{a}\left(u_{1}\right)_{a}=1 / \sqrt{1-v^{a} v_{a}}$ are the associated rapidity parameter and Lorentz factor, respectively; $\widehat{v}^{a} \equiv \widehat{v}\left(u_{2}, u_{1}\right)^{a}$ is the unit vector in the direction of $v^{a} \neq 0$ if $u_{2}^{a} \neq u_{1}^{a}$ and will be formally taken to be any unit vector in $u_{1}^{\perp}$ if $u_{2}^{a}=u_{1}^{a} \Leftrightarrow v^{a}=0 \Leftrightarrow \psi=0$, such that $v\left(u_{2}, u_{1}\right)^{a}=\tanh (\psi) \widehat{v}\left(u_{2}, u_{1}\right)^{a}$ in any case. Eq. (6) defines the unique boost $B\left(u_{2}, u_{1}\right)^{a}{ }_{b}$ which acts trivially on $\left\langle u_{1}^{a}, u_{2}^{a}\right\rangle^{\perp}$ and maps $u_{1}^{a}$ to $u_{2}^{a}=B\left(u_{2}, u_{1}\right)^{a}{ }_{b} u_{1}^{b}$; this is the identity transformation of $T_{p} M$ if $u_{2}^{a}=u_{1}^{a}$, while if $u_{2}^{a} \neq u_{1}^{a}$ one has ${ }^{3}$

$$
B\left(u_{2}, u_{1}\right)_{b}^{a} \widehat{v}\left(u_{2}, u_{1}\right)^{b}=\sinh (\psi) u_{1}^{a}+\cosh (\psi) \widehat{v}\left(u_{2}, u_{1}\right)^{a}=-\widehat{v}\left(u_{1}, u_{2}\right)^{a} .
$$

\subsection{Self-dual bivectors}

$\S 1$. Bivectors $\mathcal{X}_{a b} \in \mathbb{C} \Lambda^{2}$ that satisfy $\star \mathcal{X}_{a b}=i \mathcal{X}_{a b}$ are called self-dual; they form a $3 \mathrm{~d}$ complex vector space, denoted $S^{+}$. For any $A_{a b} \in \mathbb{C} \Lambda^{2}$ one has $A_{a b}^{\dagger} \equiv A_{a b}-i \star A_{a b} \in S^{+}$. The relations

$$
X_{a b}=\Re\left(\mathcal{X}_{a b}\right) \quad \Leftrightarrow \quad \mathcal{X}_{a b}=X_{a b}^{\dagger}=X_{a b}-i \star X_{a b}
$$

define a bijection between complex self-dual bivectors $\mathcal{X}_{a b} \in S^{+}$and real bivectors $X_{a b} \in \Lambda^{2}$, and with this notational correspondence we put

$$
I_{X} \equiv-\frac{1}{4} \mathcal{X}^{a b} \mathcal{X}_{a b}=-\frac{1}{2} X^{a b} X_{a b}+\frac{i}{2} \star X^{a b} X_{a b}
$$

For $\mathcal{X}_{a b}, \mathcal{Y}_{a b} \in S^{+}$one has the identities

$$
\begin{aligned}
& \mathcal{X}^{a b} \mathcal{Y}_{b c}+\mathcal{Y}^{a b} \mathcal{X}_{b c}=\frac{1}{2} \mathcal{X}_{g h} \mathcal{Y}^{h g} \delta_{c}^{a} \quad \Rightarrow \quad \mathcal{X}^{a}{ }_{c} \mathcal{X}^{c}{ }_{b}=I_{X} \delta_{c}^{a}, \\
& \mathcal{X}^{a}{ }_{c} \overline{\mathcal{Y}}^{c}{ }_{b}=\overline{\mathcal{Y}}^{a}{ }_{c} \mathcal{X}^{c}{ }_{b}, \quad \mathcal{X}^{a b} \overline{\mathcal{Y}}_{a b}=0 .
\end{aligned}
$$

For an arbitrary but fixed observer $u^{a} \in U^{+}$the relations

$$
x^{a}=\mathcal{X}^{a b} u_{b} \quad \Leftrightarrow \quad \mathcal{X}_{a b}=X_{a b}^{\dagger}=\left(2 u_{[a} x_{b]}\right)^{\dagger}=2 u_{[a} x_{b]}+i \epsilon_{a b c d} u^{d} x^{c}
$$

define a fundamental isometric identification of $S^{+}$and $\mathbb{C} u^{\perp}$ (pair of $3 \mathrm{~d}$ complex vector space isometries, where the restrictions of $G$ and $g$ defined in (3) are taken as respective metrics) [19: $: 4$

$$
x^{a}=\mathcal{X}^{a b} u_{b}, y^{a}=\mathcal{Y}^{a b} u_{b} \quad \Rightarrow \quad x^{a} y_{a}=-\frac{1}{4} \mathcal{X}^{a b} \mathcal{Y}_{a b}, \quad x^{a} x_{a}=I_{X} .
$$

$\S 2$. Let $\mathcal{X}_{a b}=X_{a b}^{\dagger}$ be unitary $\left(I_{X}=1\right)$. The imaginary part of $(9)$ gives $\star X^{a b} X_{a b}=0$; hence $X_{a b}$ and $\star X_{a b}$ are simple [30] and have as respective blades the orthogonal 2-planes

$$
\Sigma \equiv \operatorname{Im}\left(X_{b}^{a}\right)=\operatorname{Ker}\left(\star X_{b}^{a}\right), \quad \Sigma^{\perp}=\operatorname{Im}\left(\star X_{b}^{a}\right)=\operatorname{Ker}\left(X_{b}^{a}\right) .
$$

\footnotetext{
${ }^{3}$ The first equality in 7 is due to $B\left(u_{2}, u_{1}\right)^{a}$ being an orthochronous Lorentz transformation, while the second equality follows by reversing the roles of $u_{1}^{a}$ and $u_{2}^{a}$ in [6]; the result agrees with Eq. (14) in [26] and Eq. (4.5) in [29].

${ }^{4} \mathbb{C} u^{\perp}$ and $S^{+}$are both $3 \mathrm{~d}$ complex vector spaces; the map $x^{a} \mapsto\left(2 u_{[a} x_{b]}\right)^{\dagger}$ is clearly an injective homomorphism and thus an isomorphism, with inverse $\mathcal{X}_{a b} \mapsto \mathcal{X}^{a b} u_{b} ; 13$ follows from 10 .
} 
Combined with the real part of (9) this leads to the existence of a restricted orthonormal tetrad $\left(e_{0}^{a}, e_{i}^{a}\right)$ such that

$$
\mathcal{X}_{a b}=\left[2\left(e_{0}\right)_{[a}\left(e_{1}\right)_{b]}\right]^{\dagger}=X_{a b}-i \star X_{a b} \quad \Leftrightarrow \quad X^{a b}=2 e_{0}^{[a} e_{1}^{b]}, \quad \star X^{a b}=-2 e_{2}^{[a} e_{3}^{b]} .
$$

One has $\Sigma=\left\langle e_{0}^{a}, e_{1}^{a}\right\rangle$ and $\Sigma^{\perp}=\left\langle e_{2}^{a}, e_{3}^{a}\right\rangle$, with corresponding pair of projectors

$$
P_{b}^{a}=X_{c}^{a} X_{b}^{c}=-e_{0}^{a}\left(e_{0}\right)_{b}+e_{1}^{a}\left(e_{1}\right)_{b}, \quad P_{\perp b}^{a}=-\star X_{c}^{a} \star X_{b}^{c}=e_{2}^{a}\left(e_{2}\right)_{b}+e_{3}^{a}\left(e_{3}\right)_{b} .
$$

For any observer $u^{a}$ we have by $(12)$ and $(13)$ :

$$
\begin{aligned}
& x^{a}=\mathcal{X}^{a b} u_{b} \equiv \mathfrak{e}^{a}-i \mathfrak{b}^{a} \quad \Leftrightarrow \quad \mathfrak{e}^{a} \equiv X_{b}^{a} u^{b}, \quad \mathfrak{b}^{a} \equiv \star X_{b}^{a} u^{b} \quad \Leftrightarrow \quad X_{a b}=2 u_{[a} \mathfrak{e}_{b]}+\epsilon_{a b c d} u^{d} \mathfrak{b}^{c}, \\
& x^{a} x_{a}=\mathfrak{e}^{a} \mathfrak{e}_{a}-\mathfrak{b}^{a} \mathfrak{b}_{a}=1, \quad \mathfrak{e}^{a} \mathfrak{b}_{a}=0 .
\end{aligned}
$$

Combined with (14) it follows that $\mathfrak{b}^{a} \in \Sigma^{\perp}$ is orthogonal to the spatial, non-zero vector $\mathfrak{e}^{a} \in \Sigma$, and

$$
\mathfrak{b}^{a}=0\left(x^{a}=\mathfrak{e}^{a}\right) \quad \Leftrightarrow \quad u^{a} \in \Sigma .
$$

\section{The electromagnetic case}

\subsection{EM principal observers}

In electromagnetism the Faraday two-form $F_{a b} \in \Lambda^{2}$ is the governing tensor. Let $\mathcal{F}_{a b} \equiv F_{a b}-i \star F_{a b} \in S^{+}$ be the EM self-dual bivector. The electric and magnetic fields $E^{a}=F^{a b} u_{b}$ and $B^{a}=\star F^{a b} u_{b}$ as measured by an observer $u^{a}$ can be assembled into the complex vector $f^{a}$, which by $(12)$ determines $\mathcal{F}_{a b}$ :

$$
f^{a} \equiv E^{a}-i B^{a}=\mathcal{F}^{a b} u_{b} \quad \Leftrightarrow \quad \mathcal{F}_{a b}=2 u_{[a} f_{b]}+i \epsilon_{a b c d} u^{d} f^{c} .
$$

Referring to 9 and 13 the complex EM invariant $I_{F}$ is defined by

$$
\begin{aligned}
I_{F} & \equiv-\frac{1}{4} \mathcal{F}^{a b} \mathcal{F}_{a b}=-\frac{1}{2} F^{a b} F_{a b}+\frac{i}{2} \star F^{a b} F_{a b} \\
& =f^{a} f_{a}=E^{a} E_{a}-B^{a} B_{a}-2 i E^{a} B_{a} .
\end{aligned}
$$

The EM field is called null if $I_{F}=0$, else non-null.

Let us review the definition, characterizations, existence and locus of EM principal observers at a point $p$. The (tracefree, symmetric) EM energy-momentum tensor associated to $F_{a b}$ is given by

$$
T_{a b} \equiv \frac{1}{4 \pi} F_{a c} F_{b}{ }^{c}-\frac{1}{16 \pi} F_{c d} F^{c d} g_{a b}=\frac{1}{8 \pi} \mathcal{F}_{a c} \overline{\mathcal{F}}_{b}{ }^{c}=\frac{1}{8 \pi} \overline{\mathcal{F}}_{a c} \mathcal{F}_{b}{ }^{c} .
$$

Relative to $u^{a} \in U^{+}$the (spatial) Poynting vector $p^{a}$, energy density $\rho_{\mathrm{em}}$ and energy flux vector $s^{a}$ are

$$
\begin{aligned}
p^{a} & \equiv-h^{a b} T_{b d} u^{d}=\frac{1}{4 \pi} \epsilon_{b c d}^{a} E^{b} B^{c} u^{d} \equiv \frac{1}{4 \pi}[E, B]^{a}=\frac{1}{8 \pi i} \epsilon_{b c d}^{a} u^{d} f^{b} \bar{f}^{c} \equiv \frac{1}{8 \pi i}[f, \bar{f}]^{a}, \\
\rho_{\mathrm{em}} & \equiv T_{a b} u^{a} u^{b}=\frac{1}{8 \pi}\left(E^{a} E_{a}+B^{a} B_{a}\right)=\frac{1}{8 \pi} f^{a} \bar{f}_{a}, \\
s^{a} & \equiv-T^{a b} u_{b}=p^{a}+\rho_{\mathrm{em}} u^{a} .
\end{aligned}
$$

$T_{b}^{a}$ satisfies $T_{b}^{a} T_{c}^{b}=\chi^{2} \delta_{c}^{a}$ 31] and thus has eigenvalues $\pm \chi$, where the invariant

$$
\chi=\frac{1}{2} \sqrt{T^{a b} T_{a b}}=\frac{1}{8 \pi}\left|I_{F}\right|=\frac{1}{8 \pi}\left|f^{a} f_{a}\right|=\frac{1}{8 \pi} \sqrt{\left(E^{a} E_{a}-B^{a} B_{a}\right)^{2}+4\left(E^{a} B_{a}\right)^{2}}
$$

is the proper EM energy density [32. Combined with 26] it follows that

$$
\rho_{\mathrm{em}}^{2}=-s^{a} s_{a}+p^{a} p_{a}=\chi^{2}+p^{a} p_{a} \geq \chi^{2},
$$

such that $\chi$ is a lower bound and in fact the infimum for $\rho_{\mathrm{em}}$ regarded as a non-negative scalar function $U^{+} \rightarrow \mathbb{R}_{\geq 0}$ (i.e., if we let $u^{a}$ range over $U^{+}$) [13, 22. 
Definition 4.1. An observer $u^{a}$ is (EM) principal when it measures a vanishing Poynting vector, $p^{a}=05^{5}$

By (23)- $(28)$ an EM principal observer $u^{a}$ is characterized by any of the following conditions:

(i) the electric and magnetic fields are aligned $\left(E^{[a} B^{b]}=0\right)$, i.e., linearly dependent $[23$;

(ii) $f^{a}=\sqrt{I_{F}} \mathfrak{e}^{a}$ for some real unit vector $\mathfrak{e}^{a} \in u^{\perp}$ (cf. p. 69 of [5]);

(iii) $u^{[a} T^{b]}{ }_{c} u^{c} \equiv u^{[a} F^{b]}{ }_{c} F^{c}{ }_{d} u^{d}=0$, i.e., $u^{a}$ is an eigenvector of $T^{a}{ }_{b}$ [11, 22] or $F_{c}^{a} F_{b}^{c}$;

(iv) the EM energy density $\rho_{\mathrm{em}}$ attains minimum value, namely $\chi[13,22,26,35]$.

Note that alignment of $E^{a}$ and $B^{a}$ means $E^{a} \propto \mathfrak{e}^{a}$ and $B^{a} \propto \mathfrak{e}^{a}$, and thus $f^{a} \propto \mathfrak{e}^{a}$, for some unit vector $\mathfrak{e}^{a} \in u^{\perp}$, and so by 22 characterization (ii) is the complex version of (i).

We still mention two new characterizations, involving minimality of scalar functions $U^{+} \rightarrow \mathbb{R}_{\geq 0}$ and equivalent to (iv). First, combining (25) with the invariant $E^{a} E_{a}-B^{a} B_{a}=\Re\left(I_{F}\right)=-\frac{1}{2} F^{a b} F_{a b}$ gives

$$
E^{a} E_{a}=4 \pi \rho_{\mathrm{em}}+\frac{1}{2} \Re\left(I_{F}\right), \quad B^{a} B_{a}=4 \pi \rho_{\mathrm{em}}-\frac{1}{2} \Re\left(I_{F}\right) .
$$

Hence, if we define the invariants

$$
n_{E} \equiv \sqrt{\frac{\left|I_{F}\right|+\Re\left(I_{F}\right)}{2}}=\left|\Re\left(\sqrt{I_{F}}\right)\right|, \quad n_{B} \equiv \sqrt{\frac{\left|I_{F}\right|-\Re\left(I_{F}\right)}{2}}=\left|\Im\left(\sqrt{I_{F}}\right)\right|
$$

(where the last equalities follow from (2) applied to $z=I_{F}$ ) we conclude from characterization (iv), $\chi=\left|I_{F}\right| / 8 \pi$ and 29 that an observer is EM principal iff one of the following similar criteria holds ${ }^{6}$

(v-a) the norm $\sqrt{E^{a} E_{a}}$ attains minimum value, namely $n_{E}$;

(v-b) the norm $\sqrt{B^{a} B_{a}}$ attains minimum value, namely $n_{B}$;

Second, the (symmetric, spatial) tensor $\mathcal{T}_{a b} \equiv h_{a}{ }^{c} h_{b}{ }^{d} T_{c d}=\rho_{\mathrm{em}} h_{a b}-\frac{1}{4 \pi}\left(E_{a} E_{b}+B_{a} B_{b}\right)$ also appears in the orthogonal splitting of $T_{a b}$ relative to $u^{a}, T_{a b}=\rho_{\mathrm{em}} u_{a} u_{b}+2 u_{(a} p_{a)}+\mathcal{T}_{a b}$ [6]. One has $\mathcal{T}_{a}^{a}=\rho_{\mathrm{em}}$ and $\mathcal{T}^{a b} \mathcal{T}_{a b}=\rho_{\mathrm{em}}^{2}+2 \chi^{2}$, so an EM principal observer can also be characterized by:

(vi) $\mathcal{T}^{a b} \mathcal{T}_{a b}$ attains minimum value, namely $3 \chi^{2}$.

The existence and locus of EM principal observers can be elegantly deduced from characterization (ii): In view of 200 such observers do not exist in the null case $I_{F}=0 \Leftrightarrow \chi=0$ unless $F_{a b}=0$ at $p$, in which case all observers at $p$ are principal, trivially. The non-null case $I_{F} \neq 0$ remains. Here we can normalize $\mathcal{F}_{a b}$ to the unitary self-dual bivector $\mathcal{X}_{a b}=\mathcal{F}_{a b} / \sqrt{I_{F}}$, where $\sqrt{I_{F}}$ is one of the two square roots of $I_{F}$ given by (2) with $z=I_{F}$; by (15) a restricted orthonormal tetrad $\left(e_{0}^{a}, e_{i}^{a}\right)$ exists such that

$$
\mathcal{F}_{a b}=\sqrt{I_{F}} \mathcal{X}_{a b}, \quad \mathcal{X}^{a b} \equiv X^{a b}-i \star X^{a b}=2 e_{0}^{[a} e_{1}^{b]}+2 i e_{2}^{[a} e_{3}^{b]} .
$$

The blade $\Sigma=\left\langle e_{0}^{a}, e_{1}^{a}\right\rangle$ of $X_{a b}$ is called the timelike (EM) principal plane and equips tangent space with a $2+2$ structure $\Sigma \oplus \Sigma^{\perp}$; the two null directions of $\Sigma$ are referred to as the (EM) principal null directions (PNDs), which are the real null eigendirections (or the aligned null directions within null alignment theory [36]) of $F_{b}^{a}$ (or $X_{b}^{a}$ or $\mathcal{F}_{b}^{a}$ ), spanned by null vectors $k^{a}$ that satisfy $k^{[a} F^{b]}{ }_{c} k^{c}=0$ [22, 30]. Restricted orthonormal tetrads for which (31) holds are determined up to boosts in $\Sigma$ and rotations in $\Sigma^{\perp}$, i.e., given one such tetrad $\left(e_{0}^{a}, e_{i}^{a}\right)$ all other ones are given by

$$
\begin{array}{lcc}
E_{0}^{a}=\cosh (\gamma) e_{0}^{a}+\sinh (\gamma) e_{1}^{a}, & E_{1}^{a}=\sinh (\gamma) e_{0}^{a}+\cosh (\gamma) e_{1}^{a}, \quad \gamma \in \mathbb{R}, \\
E_{2}^{a}=\cos (\vartheta) e_{2}^{a}+\sin (\vartheta) e_{3}^{a}, & E_{3}^{a}=-\sin (\vartheta) e_{2}^{a}+\cos (\vartheta) e_{3}^{a}, \quad \vartheta \in[0,2 \pi) .
\end{array}
$$

\footnotetext{
5Such observers have been called 'observers at rest' with respect to the EM field [33]; we find however such 'rest' notion somewhat confusing, since at each point there are infinitely many principal observers, which are not at rest with respect to each other, while (according to such notion) all being at rest with respect to the field (which is odd since well posed notions of relative rest are locally transitive 34]). Therefore we shall not use it. An analogous remark applies to the same terminology used in [13] for the gravitational case in the next section.

${ }^{6}$ In [22 pp. 334-335] the invariants $n_{E}$ and $n_{B}$ were identified as the norms of the electric and magnetic fields acquired by all principal observers (and called therein the absolute electric and magnetic strengths) but not as the minimum norms.
} 
Note that an observer $E_{0}^{a} \in \Sigma$ and unit vector $E_{2}^{a} \in \Sigma^{\perp}$ determine a unique such tetrad by $E_{1}^{a}=X_{b}^{a} E_{0}^{b}$ and $E_{3}^{a}=\star X^{a}{ }_{b} E_{2}^{b}$. We could also normalize $\mathcal{F}_{a b}$ by $-\sqrt{I_{F}}$, which then yields $-\mathcal{X}_{a b}$; thus we obtain two 2-parameter families $\left(E_{0}^{a}, \pm E_{1}^{a}, E_{2}^{a}, \pm E_{3}^{a}\right)$ of (EM) principal tetrads. Contraction of (31) with an observer $u^{a}$ gives

$$
\begin{aligned}
f^{a} & =\sqrt{I_{F}} x^{a} \equiv E^{a}-i B^{a}, \quad x^{a} \equiv \mathcal{X}^{a}{ }_{b} u^{b} \equiv \mathfrak{e}^{a}-i \mathfrak{b}^{a} \\
\Leftrightarrow \quad E^{a} & =\Re\left(\sqrt{I_{F}}\right) \mathfrak{e}^{a}+\Im\left(\sqrt{I_{F}}\right) \mathfrak{b}^{a}, \quad B^{a}=-\Im\left(\sqrt{I_{F}}\right) \mathfrak{e}^{a}+\Re\left(\sqrt{I_{F}}\right) \mathfrak{b}^{a} .
\end{aligned}
$$

For given $u^{a}$ the complex vector $x^{a} \equiv f^{a} / \sqrt{I_{F}} \in \mathbb{C} u^{\perp}$ is well-defined and unit; according to characterization (ii) $u^{a}$ is EM principal precisely when $x^{a}$ is real $\left(x^{a}=\mathfrak{e}^{a}\right.$, where $\mathfrak{e}^{a}$ in (ii) and (34) are compatible). Equation 19 now shows that EM principal observers exist, and provides a characterization which may replace (ii) and describes their locus:

(ii)' the vector $x^{a} \equiv f^{a} / \sqrt{I_{F}}$ is real $\left(x^{a}=\mathfrak{e}^{a} \Leftrightarrow \mathfrak{b}^{a}=0\right)$, i.e., $u^{a}$ lies in the timelike principal plane $\Sigma$.

In summary: if an EM field is non-null at a point, the principal observers instantaneously passing through that point are precisely those belonging to the timelike principal plane, which are the unit timelike eigenvectors of $T_{b}^{a}$; for these observers, and only for these, the EM energy density is minimum and the electric and magnetic vectors are aligned and have minimum norms.

\subsection{Non-null fields: general vs. principal observers}

The EM Wheeler result [24, 5, 26] states that, given a non-principal observer $u^{a}$ in a non-null EM field, there is a (unique) principal observer $u_{\mathrm{em}}^{a}$ which travels instantaneously relative to $u^{a}$ in the direction of the Poynting vector $p^{a} \neq 0$ measured by $u^{a}$, where the relative speed $\tanh (\psi)$ between the two observers is implicitly given by (1). Geometrically this means that the 2-plane $\left\langle u^{a}, p^{a}\right\rangle$ intersects $\Sigma$ in a line (namely $\left.\left\langle u_{\mathrm{em}}^{a}\right\rangle\right)$; thus, to the arguments Wheeler gave in 24 to emphasize the "miracle" of the result we could add that two generic 2-planes in 4 dimensions only intersect in the origin.

Here we show that the EM Wheeler result is a simple consequence of the definition of the Poynting vector, and emphasize on the geometric nature of $u_{\mathrm{em}}^{a}$ and $\psi$ originating from the $2+2$ structure $\Sigma \oplus \Sigma^{\perp}$. Our analysis leads to an explicit formula for $v\left(u_{\Sigma}, u\right)^{a}$ in terms of $p^{a}$ and $\rho_{\mathrm{em}}$ and will provide a clear parallel with the Petrov type D gravitational case studied below.

To this end we first derive a lemma valid for any unitary self-dual bivector $\mathcal{X}_{a b}$. We elaborate eqs. (16)-18) and adopt the 'principal' nomenclature from the non-null EM case. Given the pair of projectors (16) any non-principal observer $u^{a} \notin \Sigma$ can be uniquely decomposed along and orthogonal to $\Sigma$ :

$$
u^{a}=P_{b}^{a} u^{b}+P_{\perp b}^{a} u^{b}=\cosh \left(\psi_{\Sigma}\right) u_{\Sigma}^{a}+\sinh \left(\psi_{\Sigma}\right) u_{\perp}^{a} .
$$

Here $u_{\Sigma}^{a} \in \Sigma$ is the observer lying along $P_{b}^{a} u^{b}, \psi_{\Sigma}=\psi\left(u, u_{\Sigma}\right)$ is the relative rapidity of $u^{a}$ and $u_{\Sigma}^{a}$, and $u_{\perp}^{a}=\widehat{v}\left(u, u_{\Sigma}\right)^{a}$ is the unit vector along $P_{\perp b}^{a} u^{b}$; in the limit case of a principal observer $\left(u^{a}=u_{\Sigma}^{a} \Leftrightarrow \psi_{\Sigma}=\right.$ $0)$ we can take any unit vector in $\Sigma^{\perp}$ for $u_{\perp}^{a}$. Eq. (36) defines a unique boost $B\left(u, u_{\Sigma}\right)^{a}{ }_{b}$ mapping $u_{\Sigma}^{a}$ to $u^{a}$, see Sec. 3.2. The inverse boost $B\left(u_{\Sigma}, u\right)^{a}{ }_{b}$ is described by

Lemma 4.2. Let $\mathcal{X}_{a b}=X_{a b}^{\dagger}$ be an arbitrary unitary self-dual bivector. For any observer $u^{a}$ consider (36) and define $\mathfrak{e}^{a}, \mathfrak{b}^{a}, x^{a}$ as in (17). Then the canonically associated observer $u_{\Sigma}^{a}=\cosh \left(\psi_{\Sigma}\right)\left[u^{a}+v\left(u_{\Sigma}, u\right)^{a}\right]$ lying along the projection of $u^{a}$ onto the principal plane $\Sigma$ (blade of $X_{a b}$ ) is given by

$$
\begin{gathered}
\cosh ^{2}\left(\psi_{\Sigma}\right)=\mathfrak{e}^{a} \mathfrak{e}_{a}=\frac{x^{a} \bar{x}_{a}+1}{2} \quad \Leftrightarrow \quad x^{a} \bar{x}_{a}=2 \mathfrak{e}^{a} \mathfrak{e}_{a}-1=\cosh \left(2 \psi_{\Sigma}\right), \\
v\left(u_{\Sigma}, u\right)^{a}=\frac{[\mathfrak{e}, \mathfrak{b}]^{a}}{\mathfrak{e}^{b} \mathfrak{e}_{b}}=\frac{[\mathfrak{e}, \mathfrak{b}]^{a}}{\cosh ^{2}\left(\psi_{\Sigma}\right)} \quad \Leftrightarrow \quad[\mathfrak{e}, \mathfrak{b}]^{a}=\frac{1}{2} \sinh \left(2 \psi_{\Sigma}\right) \widehat{v}\left(u_{\Sigma}, u\right)^{a} .
\end{gathered}
$$

Hence, when $u^{a} \notin \Sigma$ the 2-plane $\left\langle u^{a},[\mathfrak{e}, \mathfrak{b}]^{a}\right\rangle$ coincides with the plane $\left\langle u^{a}, u_{\Sigma}^{a}\right\rangle$ and intersects $\Sigma$ in $\left\langle u_{\Sigma}^{a}\right\rangle$. 
To prove this lemma we extend $u_{\Sigma}^{a}$ and $u_{\perp}^{a}$ to the principal tetrad

$$
\left(E_{0}^{a}, E_{1}^{a}, E_{2}^{a}, E_{3}^{a}\right)=\left(u_{\Sigma}^{a}, \mathfrak{e}_{\Sigma}^{a}, u_{\perp}^{a}, e_{\perp}^{a}\right)
$$

according to the note after $(33)$, such that

$$
\mathfrak{e}_{\Sigma}^{a} \equiv X_{b}^{a} u_{\Sigma}^{b}=\mathcal{X}_{b}^{a} u_{\Sigma}^{b} \equiv x_{\Sigma}^{a}, \quad e_{\perp}^{a} \equiv \star^{a} X_{b} u_{\perp}^{b}=i \mathcal{X}_{b}^{a} u_{\perp}^{b}, \quad \Sigma=\left\langle u_{\Sigma}^{a}, \mathfrak{e}_{\Sigma}^{a}\right\rangle, \Sigma^{\perp}=\left\langle u_{\perp}^{a}, e_{\perp}^{a}\right\rangle .
$$

Observe that $\mathfrak{e}_{\Sigma}^{a}$ is the vector ' $\mathfrak{e}^{a}$ ', as defined in (34) but now relative to $u_{\Sigma}^{a}$. The vectors $\mathfrak{e}^{a}$ and $\mathfrak{b}^{a}$ relative to $u^{a}$ are aligned to $\mathfrak{e}_{\Sigma}^{a}$ and $e_{\perp}^{a}$; more precisely, by substituting (36) in the second part of (17) and using $X_{b}^{a} u_{\perp}^{b}=\star X^{a}{ }_{b} u_{\Sigma}^{b}=0$ we obtain

$$
\mathfrak{e}^{a} \equiv X_{b}^{a} u^{b}=\cosh \left(\psi_{\Sigma}\right) \mathfrak{e}_{\Sigma}^{a}, \quad \mathfrak{b}^{a} \equiv \star X^{a}{ }_{b} u^{b}=\sinh \left(\psi_{\Sigma}\right) e_{\perp}^{a} .
$$

This implies $\mathfrak{e}^{a} \mathfrak{e}_{a}=\cosh ^{2}\left(\psi_{\Sigma}\right), \mathfrak{b}^{a} \mathfrak{b}_{a}=\sinh ^{2}\left(\psi_{\Sigma}\right)$, which confirms 18 and together with $x^{a} \bar{x}_{a}=$ $\mathfrak{e}^{a} \mathfrak{e}_{a}+\mathfrak{b}^{a} \mathfrak{b}_{a}$ gives (37). The boost $B\left(u, u_{\Sigma}\right)^{a}{ }_{b}$ transforms (39) to the restricted orthonormal tetrad $\left(u^{a}, \mathfrak{e}_{\Sigma}^{a},-\left[\mathfrak{e}_{\Sigma}, e_{\perp}\right]^{a}, e_{\perp}^{a}\right)$, where

$$
\left[\mathfrak{e}_{\Sigma}, e_{\perp}\right]^{a} \equiv \epsilon_{b c d}^{a} \mathfrak{e}_{\Sigma}^{b} e_{\perp}^{c} u^{d}=-\left[\sinh \left(\psi_{\Sigma}\right) u_{\Sigma}^{a}+\cosh \left(\psi_{\Sigma}\right) u_{\perp}^{a}\right] .
$$

If $u^{a} \neq u_{\Sigma}^{a}$ then (7) applied to $u_{1}^{a}=u_{\Sigma}^{a}, u_{2}^{a}=u^{a}$ and $\widehat{v}\left(u, u_{\Sigma}\right)^{a}=u_{\perp}^{a}$ gives

$$
\widehat{v}\left(u_{\Sigma}, u\right)^{a}=\left[\mathfrak{e}_{\Sigma}, e_{\perp}\right]^{a},
$$

and when combined with (41) this implies (38). If $u^{a}=u_{\Sigma}^{a}$ we have $v\left(u_{\Sigma}, u\right)^{a}=0, \psi_{\Sigma}=0$ and $\mathfrak{b}^{a}=0$ (where (41) confirms (19)) and may formally take any unit vector in $u^{\perp}$ for $\widehat{v}\left(u_{\Sigma}, u\right)^{a}$. This proves the lemma. An alternative proof goes by combining the first part of (16) with (17), giving

$$
\cosh \left(\psi_{\Sigma}\right) u_{\Sigma}^{a}=P_{b}^{a} u^{b}=X_{c}^{a}{ }_{c}{ }^{c}{ }_{b} u^{c}=X^{a}{ }_{b}{ }^{b}=\mathfrak{e}^{b} \mathfrak{e}_{b} u^{a}+[\mathfrak{e}, \mathfrak{b}]^{a},
$$

which immediately implies (37)-38).

We now apply the above to non-null EM fields. By its definition 24 the Poynting vector associated to $u^{a}$ is a multiple of the vector product of $E^{a}$ and $B^{a}$ (or of $f^{a}$ and $f$ ) and thus of $\mathfrak{e}^{a}$ and $\mathfrak{b}^{a}$ by (35) (or by (34) and $[x, \bar{x}]^{a}=2 i[\mathfrak{e}, \mathfrak{b}]^{a}$ ); using also 27) we obtain

$$
p^{a} \equiv \frac{1}{4 \pi}[E, B]^{a}=\frac{1}{8 \pi i}[f, \bar{f}]^{a}=\frac{\left|I_{F}\right|}{8 \pi i}[x, \bar{x}]^{a}=2 \chi[\mathfrak{e}, \mathfrak{b}]^{a} .
$$

Lemma 4.2 now confirms the EM Wheeler result and implies that $u_{\mathrm{em}}^{a}=u_{\Sigma}^{a}$ and $\psi=\psi_{\Sigma}$ in (1): For $u^{a} \notin \Sigma$ the 2-plane $\left\langle u^{a}, p^{a}\right\rangle$ equals $\left\langle u^{a}, u_{\Sigma}^{a}\right\rangle$ and intersects $\Sigma$ in $\left\langle u_{\mathrm{em}}^{a}\right\rangle=\left\langle u_{\Sigma}^{a}\right\rangle$; by (38, 42, 43) one has

$$
\begin{aligned}
p^{a} & =2 \chi \cosh ^{2}\left(\psi_{\Sigma}\right) v\left(u_{\Sigma}, u\right)^{a} \\
& =\chi \sinh \left(2 \psi_{\Sigma}\right) \widehat{v}\left(u_{\Sigma}, u\right)^{a}=-\chi \sinh \left(2 \psi_{\Sigma}\right)\left[\sinh \left(\psi_{\Sigma}\right) u_{\Sigma}^{a}+\cosh \left(\psi_{\Sigma}\right) u_{\perp}^{a}\right] .
\end{aligned}
$$

Analogously, substituting (34) in the final expression of (25) and using (27), (37) it follows that

$$
\rho_{\mathrm{em}}=\chi x^{a} \bar{x}_{a}=\chi \cosh \left(2 \psi_{\Sigma}\right)=2 \chi \cosh ^{2}\left(\psi_{\Sigma}\right)-\chi=\chi+2 \chi \sinh ^{2}\left(\psi_{\Sigma}\right) .
$$

Comparing (46) with 48) gives $v\left(u_{\Sigma}, u\right)^{a}$ explicitly in terms of $p^{a}$ and $\rho_{\mathrm{em}}$, as announced, and we obtain

Theorem 4.3 (Extended EM Wheeler result). Suppose the EM field is non-null at a point p, and let $u^{a}$ be an observer measuring a Poynting vector $p^{a}$ and energy density $\rho_{\mathrm{em}}$ at $p$. Then there is a principal observer instantaneously traveling relative to $u^{a}$ in the direction of $p^{a}$, namely the observer $u_{\Sigma}^{a}$ lying along the orthogonal projection of $u^{a}$ onto the EM principal plane $\Sigma$, with spatial velocity

$$
v\left(u_{\Sigma}, u\right)^{a}=v_{\| p}^{a} \equiv \frac{p^{a}}{\chi\left(\zeta_{\mathrm{em}}+1\right)}=\frac{p^{a}}{\rho_{\mathrm{em}}+\chi}, \quad \zeta_{\mathrm{em}}=\frac{\rho_{\mathrm{em}}}{\chi}=\cosh \left(2 \psi_{\Sigma}\right) .
$$

Hence

$$
u_{\Sigma}^{a}=\cosh \left(\psi_{\Sigma}\right)\left[u^{a}+v_{\| p}^{a}\right], \quad \cosh \left(\psi_{\Sigma}\right)=\sqrt{\frac{\zeta_{\mathrm{em}}+1}{2}}=\sqrt{1+\frac{\rho_{\mathrm{em}}-\chi}{2 \chi}} .
$$


Remark 4.4. An alternative proof of theorem 4.3, specific to the non-null EM case, goes by substituting (31) into 23), and invoking (27) and $P_{\perp b}^{a}=\delta_{b}^{a}-P_{b}^{a}$, which gives (cf. [13, 22])

$$
T^{a}{ }_{b}=\frac{1}{8 \pi} \mathcal{F}^{a c} \overline{\mathcal{F}}_{b c}=\chi\left(P_{\perp b}^{a}-P_{b}^{a}\right)=\chi\left(\delta_{b}^{a}-2 P_{b}^{a}\right) .
$$

Note that the $2+2$ structure $\Sigma \oplus \Sigma^{\perp}$ of tangent space embodied in the basic field $\mathcal{F}_{a b}$ has carried over to the endomorphism $T^{a}{ }_{b}$ of $T_{p} M$, which is diagonalizable and has two double eigenvalues $-\chi, \chi$ with respective $2 \mathrm{~d}$ eigenspaces $\Sigma, \Sigma^{\perp}$ and projectors $P_{b}^{a}, P_{\perp b}^{a}$. Contraction of (51) with $u^{b}$ and use of (26) directly leads to $(49)-(50)$.

Remark 4.5. Making the identification (39) in (32)-(33) and (36) one gets a full decomposition of any observer $u^{a}$ in an arbitrary but fixed principal tetrad $\left(e_{0}^{a}, e_{i}^{a}\right)$ :

$$
u^{a}=\cosh \left(\psi_{\Sigma}\right)[\underbrace{\cosh (\gamma) e_{0}^{a}+\sinh (\gamma) e_{1}^{a}}_{u_{\Sigma}^{a}=E_{0}^{a}(\gamma)}]+\sinh \left(\psi_{\Sigma}\right) \underbrace{\left[\cos (\vartheta) e_{2}^{a}+\sin (\vartheta) e_{3}^{a}\right]}_{u_{\perp}^{a}=E_{2}^{a}(\vartheta)} .
$$

This manifests the intrinsic structure of $U^{+}$(observer geometry) induced by the $2+2$ structure $\Sigma \oplus \Sigma^{\perp}$ of tangent space, where an observer $u^{a}$ is fully determined by the projected principal observer $u_{\Sigma}^{a}=E_{0}^{a}(\gamma) \in$ $\Sigma$ (parameter $\gamma \in \mathbb{R})$, the relative rapidity parameter $\psi_{\Sigma}=\psi\left(u, u_{\Sigma}\right) \geq 0$ and, when $u^{a} \notin \Sigma \Leftrightarrow \psi_{\Sigma}>0$, by the direction $u_{\perp}^{a}=\widehat{v}\left(u, u_{\Sigma}\right)=E_{2}^{a}(\vartheta) \in \Sigma^{\perp}$ in which it moves relative to $u_{\Sigma}^{a}$ (parameter $\vartheta \in[0,2 \pi[$ ). By (47), (48), (27) and (29)-(30) the EM energy density and the norms of the Poynting, electric and magnetic vectors only depend on $\psi_{\Sigma}$ (and not on $\gamma$ nor $\vartheta$ ):

$$
\begin{aligned}
& \sqrt{p^{a} p_{a}}=\chi \sinh \left(2 \psi_{\Sigma}\right), \quad \rho_{\mathrm{em}}=\chi \cosh \left(2 \psi_{\Sigma}\right), \\
& E^{a} E_{a}-n_{E}^{2}=B^{a} B_{a}-n_{B}^{2}=\left|I_{F}\right| \sinh ^{2}\left(\psi_{\Sigma}\right) .
\end{aligned}
$$

From this perspective the Wheeler equation (1) with $\psi=\psi_{\Sigma}$ just expresses the ratio of the two functions (53) of the single variable $2 \psi_{\Sigma}$. For large values of $\psi_{\Sigma}$ all scalars (53)-(54) quadratically increase as functions of the Lorentz factor $\cosh \left(\psi_{\Sigma}\right)$ between $u^{a}$ and $u_{\Sigma}^{a}$, where $\sqrt{p^{a} p_{a}} / \rho_{\mathrm{em}} \rightarrow 1$ and $E^{a} E_{a} / B^{b} B_{b} \rightarrow 1$ in the limit $\psi_{\Sigma} \rightarrow \infty$ where the observer's velocity approaches the speed of light. The value $\psi_{\Sigma}=0$ corresponds to principal observers $u^{a}=u_{\Sigma}^{a}$, for which the minimum values are acquired (see the definition and characterizations (iv) and (v) of principal observers).

Within the observer geometry the principal observers are written as $E_{0}^{a}(\gamma), \gamma \in \mathbb{R}$ and form the curve $U^{+} \cap \Sigma$. This curve naturally foliates $U^{+}$, each 2 d leaf $\mathcal{L}(\gamma)$ corresponding to a fixed $\gamma \in \mathbb{R}$ (so consisting of all observers $u^{a}$ who have $E_{0}^{a}(\gamma)$ as their projected principal observer $\left.u_{\Sigma}^{a}\right)$ and parametrized by $\psi_{\Sigma}$ and $\vartheta$. Take any $u^{a} \in \mathcal{L}(\gamma)$ and consider the corresponding vector $\mathfrak{e}^{a} \neq 0$. From (34)-(35), (40), (41) and characterizations (i) and (ii)' of EM principal observers, we see that $\mathfrak{e}^{a}$ is parallel to $\mathfrak{e}_{\Sigma}^{a}=E_{1}^{a}(\gamma)=\sinh (\gamma) e_{0}^{a}+\cosh (\gamma) e_{1}^{a}$ (its norm $\cosh \left(\psi_{\Sigma}\right)$ being moreover independent of $\vartheta$ ) and thus gives the direction of the aligned fields $E_{\Sigma}^{a}=\Re\left(\sqrt{I_{F}}\right) \mathfrak{e}_{\Sigma}^{a}$ and $B_{\Sigma}^{a}=-\Im\left(\sqrt{I_{F}}\right) \mathfrak{e}_{\Sigma}^{a}$ corresponding to $u_{\Sigma}^{a}=E_{0}^{a}(\gamma)$; hence it lies along the unique spatial direction of $\Sigma$ orthogonal to $u^{a}$ or $u_{\Sigma}^{a}$. If moreover $u^{a} \neq u_{\Sigma}^{a}$ then, by the extended EM Wheeler result, the 2-plane $\left\langle u^{a}, p^{a}\right\rangle$ intersects $\mathcal{L}(\gamma)$ in a curve containing $u_{\Sigma}^{a}$, which separates it in two parts corresponding to $\vartheta=\vartheta\left(u^{a}\right)$ and $\vartheta \pm \pi$ and consisting of the non-principal observers who instantaneously travel relative to $u_{\Sigma}^{a}$ in the same $\left(u_{\perp}^{a}=E_{2}^{a}(\vartheta)\right)$ or opposite $\left(-u_{\perp}^{a}\right)$ direction as $u^{a}$ or, equivalently, who have Poynting vectors lying in $\left\langle u^{a}, p^{a}\right\rangle$. This last equivalence is due to (47) and (52), which moreover imply that the relation between non-principal observers $u^{a} \in U^{+} \backslash \Sigma$ and measured Poynting vectors is one-to-one: the projections of a given $0 \neq p^{a} \in T_{p} M$ along and orthogonal to $\Sigma$ normalize to $-u_{\Sigma}^{a}$ and $-u_{\perp}^{a}$, which yields $\gamma$ and $\vartheta$ in the decomposition (52), while by (53) the norm of $p^{a}$ yields $\psi_{\Sigma}$; since $\left(u^{a}, e^{a}, b^{a}, p^{a}\right)$ is a basis of $T_{p} M$ we also have

$$
\tilde{u}^{a} \in\left\langle u^{a}, p^{a}\right\rangle \Leftrightarrow \tilde{u}^{a} x_{a}=0 \Leftrightarrow \tilde{u}^{a} \mathcal{X}_{a b} u^{b}=0 \Leftrightarrow \tilde{u}^{a} \mathcal{F}_{a b} u^{b}=0,
$$

which for $\tilde{u}^{a} \notin \Sigma$ is equivalent to $u^{a} \in\left\langle\tilde{u}^{a}, \tilde{p}^{a}\right\rangle$ and else to $\tilde{u}^{a}=u_{\Sigma}^{a}$. On the other hand, taking $\gamma$ and $\psi_{\Sigma}>0$ fixed gives a circle of observers; gluing these circles by letting $\gamma$ run through $\mathbb{R}$ produces the $2 \mathrm{~d}$ level surface of observers measuring the same energy density $\rho_{\mathrm{em}}=\rho_{\mathrm{em}}\left(\psi_{\Sigma}\right)$ (or the same norm of the Poynting or electric or magnetic vector). 


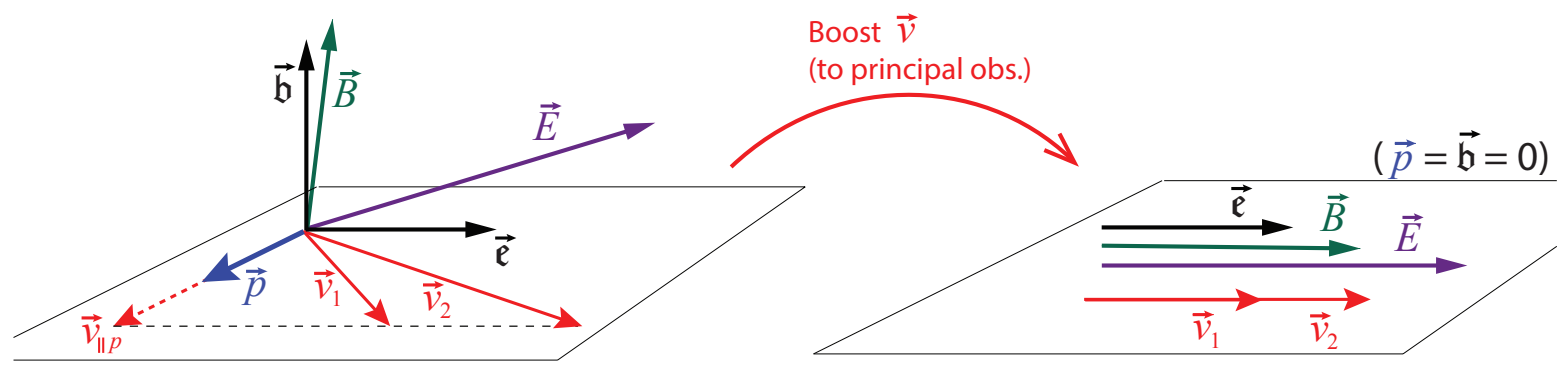

Figure 1: Velocities of the principal observers of the electromagnetic field, represented in the rest space of a generic observer $u^{a}$ (measuring fields $E^{a}, B^{a}, \mathfrak{e}^{a}, \mathfrak{b}^{a}$, and a Poynting vector $p^{a}$ ). They move with a relative velocity $v^{a}$ that has a fixed component $v_{\| p}^{a} \equiv p^{a} /\left(\rho_{\mathrm{em}}+\chi\right)$ along $p^{a}$, and a 'free' component $v_{\| \mathrm{e}}^{a}$ along the orthogonal vector $\mathfrak{e}^{a}$. When $u^{a}$ is principal (right panel), the electric and magnetic fields $E^{a}$ and $B^{a}$ are aligned along $\mathfrak{e}^{a}$, and all other principal observers are obtained by boosting along $\left\langle\mathfrak{e}^{a}\right\rangle$ with arbitrary rapidity.

Consider an observer $u^{a}$ and the $\Sigma$-projected principal observer $u_{\Sigma}^{a}$. By (40) and (41) all principal observers, i.e., the vectors $u^{\prime a} \in U^{+} \cap \Sigma$ are obtained by arbitrarily boosting $u_{\Sigma}^{a}$ along $\left\langle\mathfrak{e}_{\Sigma}^{a}\right\rangle=\left\langle\mathfrak{e}^{a}\right\rangle$, so

$$
u^{\prime a}=\cosh (\varphi) u_{\Sigma}^{a}+\sinh (\varphi) \mathfrak{e}_{\Sigma}^{a} \quad \Leftrightarrow \quad v\left(u^{\prime}, u_{\Sigma}\right)^{a}=\tanh (\varphi) \mathfrak{e}_{\Sigma}^{a}=\frac{\tanh (\varphi)}{\cosh \left(\psi_{\Sigma}\right)} \mathfrak{e}^{a}, \quad \varphi \in \mathbb{R} .
$$

In passing, note that the (unit) vector $\mathfrak{e}^{\prime a}$ along the aligned fields $E^{\prime a}=\Re\left(\sqrt{I_{F}}\right) \mathfrak{e}^{\prime a}$ and $B^{\prime a}=-\Im\left(\sqrt{I_{F}}\right) \mathfrak{e}^{\prime a}$ as measured by $u^{\prime a}$ is given by [see the primed versions of (17) and (35), with $\mathfrak{b}^{\prime a}=0$, and use (15) with $e_{0}^{a}=u_{\Sigma}^{a}$ and $\left.e_{1}^{a}=\mathfrak{e}_{\Sigma}^{a}\right]$

$$
\mathfrak{e}^{\prime a}=X^{a b} u_{b}^{\prime}=\cosh (\varphi) \mathfrak{e}_{\Sigma}^{a}+\sinh (\varphi) u_{\Sigma}^{a}
$$

By (56) the vector $v\left(u^{\prime}, u_{\Sigma}\right)^{a}$ is orthogonal to $u^{a}$, such that the velocities of the principal observers relative to $u^{a}$ are $v\left(u^{\prime}, u\right)^{a}=v\left(u_{\Sigma}, u\right)^{a}+v\left(u^{\prime}, u_{\Sigma}\right)^{a} / \cosh \left(\psi_{\Sigma}\right)$; by (49)-50 and (56) they are thus the orthogonal sum of a fixed component along $p^{a}$, and a 'free' component along $\left\langle\mathfrak{e}^{a}\right\rangle$ parametrized by $\varphi$ :

$$
v^{a} \equiv v\left(u^{\prime}, u\right)^{a}=v_{\| p}^{a}+v_{\| \mathfrak{e}}^{a}, \quad v_{\| \mathfrak{e}}^{a}=\tanh (\varphi) \frac{2 \mathfrak{e}^{a}}{\zeta_{\mathrm{em}}+1}, \varphi \in \mathbb{R}=\text { 'free' vector along }\left\langle\mathfrak{e}^{a}\right\rangle,
$$

with associated Lorentz factor $7^{7} \cosh (\psi)=\cosh (\varphi) \cosh \left(\psi_{\Sigma}\right)=\cosh (\varphi) \sqrt{\left(\zeta_{\mathrm{em}}+1\right) / 2}$; when $u^{a}$ is itself principal $\left(p^{a}=0\right)$ both $E^{a}$ and $B^{a}$ are aligned with $\mathfrak{e}^{a}$. This is illustrated in Fig. 1. The vector $\mathfrak{e}^{a}$ can be calculated by using (34) and applying (2) to $z=I_{F}$, giving

$$
\mathfrak{e}^{a}=\Re\left(\frac{f^{a}}{\sqrt{I_{F}}}\right)=\frac{1}{\left|I_{F}\right|}\left(\sqrt{\frac{\left|I_{F}\right|+\Re\left(I_{F}\right)}{2}} E^{a}-\operatorname{sgn}\left(\Im\left(I_{F}\right)\right) \sqrt{\frac{\left|I_{F}\right|-\Re\left(I_{F}\right)}{2}} B^{a}\right) .
$$

Thus, an observer $u^{a}$ passing through a point $p$ and measuring $f^{a}=E^{a}-i B^{a}$ may check whether the EM field is non-null at $p$, and in this case determine all principal observers directly from $f^{a}$, by the following

Algorithm for obtaining the principal observers from measured electric and magnetic fields:

1. Compute $I_{F}=f^{a} f_{a}$; if $I_{F} \neq 0$ the EM field is non-null at $p$, and then go to step 2 .

2. Compute $p^{a}, \rho_{\mathrm{em}}$ and $\chi$ from (24), 25) and (27); then (49) gives the velocity relative to $u^{a}$ of the projected principal observer $u_{\Sigma}^{a}$ realizing the EM Wheeler result.

\footnotetext{
${ }^{7}$ The Lorentz factor is thus minimal for $u^{\prime a}=u_{\Sigma}^{a} \Leftrightarrow \varphi=0$, cf. the appendix of [24]; note that Eq. (36) in [24] is equivalent to $\cosh (\beta)=\cosh (\mu) \cosh (\alpha)$, where $(\beta, \mu, \alpha)$ corresponds to our $\left(\psi, \varphi, \psi_{\Sigma}\right)$.
} 
3. Compute $\mathfrak{e}^{a}$ from (58); then $(49)$ and (57) give the velocities relative to $u^{a}$ of all principal observers $u^{\prime a}$.

Remark 4.6. Let $I_{F} \neq 0$ and define $\sigma \in\left[0, \pi\left[\right.\right.$ by $e^{2 i \sigma}=I_{F} /\left|I_{F}\right|$. By (35) and their characterization (ii)' [and see (i)] the principal observers are precisely those for which $\cos (\sigma) B^{a}+\sin (\sigma) E^{a}=0$. By 21 the condition $\Im\left(I_{F}\right)=0$ means that $E^{a}$ and $B^{a}$ are orthogonal for any observer $\left(E^{a} B_{a}=0\right)$; this gives purely electric (PE) and purely magnetic (PM) electromagnetic fields, characterized by $\sigma=0 \Leftrightarrow I_{F}=$ $\left|I_{F}\right|>0 \Leftrightarrow E^{a} E_{a}>B^{a} B_{a}$ and $\sigma=\pi / 2 \Leftrightarrow I_{F}=-\left|I_{F}\right|<0 \Leftrightarrow E^{a} E_{a}<B^{a} B_{a}$, respectively, and the principal observers are precisely those for which the magnetic (electric) field vanishes. In (49)- 58 we then have

$$
\begin{aligned}
& \mathrm{PE}: \quad v_{\| p}^{a}=[E, B]^{a} / E^{b} E_{b}, \quad \mathfrak{e}^{a}=E^{a} / \sqrt{E^{b} E_{b}-B^{b} B_{b}} \text {, } \\
& \mathrm{PM}: \quad v_{\| p}^{a}=[E, B]^{a} / B^{b} B_{b}, \quad \mathfrak{e}^{a}=B^{a} / \sqrt{B^{b} B_{b}-E^{b} E_{b}},
\end{aligned}
$$

in agreement with 37. Note that a real bivector that has a unitary self-dual bivector is 'purely electric', with $E^{a}=\mathfrak{e}^{a}, B^{a}=\mathfrak{b}^{a}[$ compare (44) with $(59)]$; in general, $\tilde{F}_{a b}=\left(\cos (\sigma) F_{a b}-\sin (\sigma) \star F_{a b}\right) / \sqrt{\left|I_{F}\right|}$ is such a bivector that is canonically obtained from a non-null EM field $F_{a b}$ by duality rotation over $\sigma$ and normalization, and the associated electric field $\tilde{E}^{a}=\tilde{F}^{a b} u_{b}$ precisely equals the vector (58).

\section{The gravitational case}

\subsection{Weyl principal observers}

Consider the Weyl tensor $C_{a b c d}$ at a spacetime point $p$. One has $\frac{1}{2} \epsilon_{a b e f} C_{c d}^{e f}=\frac{1}{2} C_{a b}{ }^{e f} \epsilon_{c d e f} \equiv \star C_{a b c d}$ and defines the self-dual Weyl tensor by $\mathcal{C}_{a b c d} \equiv C_{a b c d}-i \star C_{a b c d}$. Given any observer $u^{a}$ the electric and magnetic parts $E_{a b} \equiv C_{a c b d} u^{c} u^{d}$ and $H_{a b} \equiv \star C_{a c b d} u^{c} u^{d}$ of the Weyl tensor relative to $u^{a}$ are spatial, traceless and symmetric, and assembled into the complex tensor

$$
Q_{a b} \equiv \mathcal{C}_{a c b d} u^{c} u^{d}=C_{a c b d} u^{c} u^{d}-i \star C_{a c b d} u^{c} u^{d}=E_{a b}-i H_{a b}
$$

Conversely, one has 19

$$
\mathcal{C}_{a b}{ }^{c d}=4\left(u_{[\alpha} u^{[c}+h_{[a}^{[c}\right) Q_{b]}^{d]}-2 i \epsilon_{a b e f} u^{f} Q^{e[c} u^{d]}-2 i \epsilon^{c d e f} u_{f} Q_{e[a} u_{b]}
$$

This implies that the induced traceless endomorphism $\mathrm{Q}=\mathrm{E}-i \mathrm{H}: v^{a} \mapsto Q^{a}{ }_{b} v^{b}$ of $\mathbb{C} u^{\perp}$ is associated to the traceless endomorphism $\mathrm{C}: \mathcal{X}_{a b} \mapsto-\frac{1}{4} \mathcal{C}_{a b} c d \mathcal{X}_{c d}$ of $S^{+}$under the identification (12) of $S^{+}$and $\mathbb{C} u^{\perp}$, in the sense that if the isomorphism $\mathcal{X}_{a b} \mapsto \mathcal{X}^{a b} u_{b}$ is denoted by $\psi_{u}$ then

$$
\mathrm{Q}=\psi_{u} \circ \mathrm{C} \circ \psi_{u}^{-1}
$$

Thus, for any $u^{a}, \mathrm{Q}$ has the same eigenvalues and algebraic type as C. The eigenvalues $\lambda_{k}(k=1,2,3)$ sum to zero and solve the joint characteristic equation $x^{3}-\frac{1}{2} I x-\frac{1}{3} J=0$, where $I$ and $J$ are the complex quadratic and cubic Weyl invariants

$$
\begin{aligned}
I & \equiv \operatorname{tr}\left(\mathrm{C}^{2}\right)=\frac{1}{16} \mathcal{C}_{a b}{ }^{c d} \mathcal{C}_{c d}{ }^{a b}=\frac{1}{8} C_{a b}{ }^{c d}\left(C_{c d}{ }^{a b}-i \star C_{c d}{ }^{a b}\right)=\sum_{k=1}^{3} \lambda_{k}^{2}=-2 \sum_{(i j k)} \lambda_{j} \lambda_{k} \\
& =\operatorname{tr}\left(\mathrm{Q}^{2}\right)=Q^{a}{ }_{b} Q^{b}{ }_{a}=E^{a}{ }_{b} E^{b}{ }_{a}-H^{a}{ }_{b} H^{b}{ }_{a}-2 i E_{b}^{a} H^{b}{ }_{a}, \\
J & \equiv \operatorname{tr}\left(\mathrm{C}^{3}\right)=-\frac{1}{64} \mathcal{C}_{a b}{ }^{c d} \mathcal{C}_{c d}{ }^{e f} \mathcal{C}_{e f}{ }^{a b}=-\frac{1}{16} C_{a b}{ }^{c d} C_{c d}{ }^{e f}\left(C_{e f}{ }^{a b}-i \star C_{e f}{ }^{a b}\right)=\sum_{k=1}^{3} \lambda_{k}^{3}=3 \prod_{k=1}^{3} \lambda_{k} \\
& =\operatorname{tr}\left(\mathrm{Q}^{3}\right)=Q^{a}{ }_{b} Q^{b}{ }_{c} Q^{c}{ }_{a}=E^{a}{ }_{b} E^{b}{ }_{c} E^{c}{ }_{a}-3 E^{a}{ }_{b} H_{c}^{b} H^{c}{ }_{a}+i\left(H^{a}{ }_{b} H^{b}{ }_{c} H^{c}{ }_{a}-3 E_{b}^{a} E_{c}^{b} H_{a}^{c}\right) .
\end{aligned}
$$

There are six algebraic types, known as the Petrov types (see e.g. [19], and appendix B.1): 
- Petrov type $I$ is the 'algebraically general' case $I^{3} \neq 6 J^{2}$, with three simple eigenvalues.

- Petrov types II and D both have $I^{3}=6 J^{2} \neq 0$ and thus one double eigenvalue $\lambda \equiv-J / I$ and one simple eigenvalue $-2 \lambda$, but the minimal polynomial is $(x-\lambda)^{2}(x+2 \lambda)$ for Petrov type II while $(x-\lambda)(x+2 \lambda)$ for Petrov type $\mathrm{D}$, so in the latter case $Q_{b}^{a}$ (relative to any $u^{a}$ ) satisfies

$$
\left(Q_{c}^{a}-\lambda h_{c}^{a}\right)\left(Q_{b}^{c}+2 \lambda h_{b}^{c}\right)=0, \quad \lambda \equiv-J / I
$$

- Petrov types III, $\mathrm{N}$ and $\mathrm{O}$ have $I=J=0$ and thus a triple eigenvalue 0 , but the minimal polynomials are $x^{3}, x^{2}$ and $x$, respectively; Petrov type $\mathrm{O}$ is thus the trivial case where $C_{a b c d}=0$.

As for any endomorphism on a vector space, $\mathrm{C}$ and $\mathrm{Q}$ are diagonalizable precisely when their (joint) minimal polynomial only has linear factors, i.e., when its degree equals the number of different eigenvalues; this is the case for Petrov types I, D and O but not for types II, III and N.

Akin to the EM case we review the definition, characterizations, existence and locus of Weyl principal observers. The basic super-energy tensor of the Weyl tensor is the (completely symmetric, tracefree) Bel-Robinson tensor 1, $3{ }^{8}$

$$
T_{a b c d} \equiv \frac{1}{4} \mathcal{C}_{a}{ }^{e}{ }_{c}^{f} \overline{\mathcal{C}}_{b e d f}=\frac{1}{2} C_{a}{ }^{e}{ }_{(c}{ }^{f} C_{d) f b e}-\frac{1}{32} C^{e f g h} C_{e f g h} g_{a b} g_{c d} .
$$

Following [13, 38, we define the invariants

$$
\alpha \equiv \frac{1}{2} \sqrt{T^{a b c d} T_{a b c d}}=\frac{1}{2}|I|, \quad \xi \equiv \frac{1}{4} \sum_{i=1}^{3}\left|\lambda_{i}\right|^{2}, \quad q \equiv \Im\left(\lambda_{1} \bar{\lambda}_{2}\right)=\Im\left(\lambda_{2} \bar{\lambda}_{3}\right)=\Im\left(\lambda_{3} \bar{\lambda}_{1}\right) .
$$

As shown in appendix B.2 these are related by the identity

$$
4 \xi^{2}=\alpha^{2}+3 q^{2} .
$$

Relative to an arbitrary observer $u^{a}$ the (spatial) super-Poynting vector $\mathcal{P}^{a}$, super-energy density $\rho_{\mathrm{g}}$ and super-energy flux vector $\mathcal{S}^{a}$ are defined by

$$
\begin{aligned}
\mathcal{P}^{a} & \equiv-h^{a b} T_{b c d e} u^{c} u^{d} u^{e}=\frac{1}{2} \epsilon_{b c d}^{a} E^{b}{ }_{e} H^{e c} u^{d} \equiv \frac{1}{2}[\mathrm{E}, \mathrm{H}]^{a}=\frac{1}{4 i} \epsilon_{b c d}^{a} Q^{b}{ }_{e} \bar{Q}^{e c} u^{d} \equiv \frac{1}{4 i}[\mathrm{Q}, \overline{\mathrm{Q}}]^{a}, \\
\rho_{\mathrm{g}} & \equiv-\mathcal{S}^{a} u_{a}=T_{a b c d} u^{a} u^{b} u^{c} u^{d}=\frac{1}{4}\left(E^{a b} E_{a b}+H^{a b} H_{a b}\right)=\frac{1}{4} Q^{a b} \bar{Q}_{a b}=\frac{1}{4} \operatorname{tr}(\overline{\mathrm{Q}}), \\
\mathcal{S}^{a} & \equiv-T^{a b c d} u_{b} u_{c} u_{d}=\rho_{\mathrm{g}} u^{a}+\mathcal{P}^{a} .
\end{aligned}
$$

In [13, 39] it was proven that

$$
\mathcal{P}^{a}=0 \Rightarrow \rho_{\mathrm{g}}=\xi, \quad \rho_{\mathrm{g}}^{2} \geq \rho_{\mathrm{g}}^{2}-\mathcal{P}^{a} \mathcal{P}_{a}=-\mathcal{S}^{a} \mathcal{S}_{a} \geq \xi^{2} \geq \frac{1}{4} \alpha^{2},
$$

and that $\xi$ is the infimum for $\rho_{\mathrm{g}}: U^{+} \rightarrow \mathbb{R}_{\geq 0}$, called the proper gravitational super-energy density.

Definition 5.1. An observer $u^{a}$ is (Weyl) principal if the relative super-Poynting vector vanishes, $\mathcal{P}^{a}=0$.

The following equivalent properties characterize a Weyl principal observer $u^{a}$ :

(i) the associated endomorphisms $\mathrm{E}$ and $\mathrm{H}$ commute, $[\mathrm{E}, \mathrm{H}]^{a}=0[12$;

(ii) $Q$ is diagonalizable and admits real orthonormal eigenvectors $\mathfrak{e}_{i}^{a} \in u^{\perp}: Q^{a b}=\sum_{i=1}^{3} \lambda_{i} \mathfrak{e}_{i}^{a} \mathfrak{e}_{i}^{b}$ [19];

(iii) the observer $u^{a}$ satisfies $u^{[a} T^{b]} c d e u^{c} u^{d} u^{e} \equiv u^{[a} C^{b] f g}{ }_{e} C_{c f d g} u^{c} u^{d} u^{e}=0$;

(iv) the super-energy density $\rho_{\mathrm{g}}$ attains minimum value, namely $\xi[13,39$.

\footnotetext{
${ }^{8}$ The definition 69. matches the one in e.g. [13]; it has a factor $1 / 4$ compared to the one in [3], whereas the original Bel-Robinson tensor has a factor 1/2 1]; as a result different conventions for super-energy density and super-Poynting vector have been used in the literature.
} 
Characterizations (i) and (iii) follow from $(72)$ and 74 , (69). Since $E_{a b}$ and $H_{a b}$ are spatial and symmetric, commutation of $E$ and $H$ precisely means that $E$ and $H$, and thus also $Q$, can be simultaneously diagonalized in a real orthonormal frame of $\mathbb{C} u^{\perp}$, such that (i) is equivalent to (ii). Finally, if $\rho_{\mathrm{g}}$ attains minimum value then it should equal the infimum $\xi$, in which case the inequalities in the middle part of 755 become equalities, implying $\mathcal{P}^{a}=0$; this and the first part of 75 prove characterization (iv) $9^{9}$

Analogously to the EM case we note on additional characterizations, involving minimality of scalar functions $U^{+} \rightarrow \mathbb{R}_{\geq 0}$ and equivalent to (iv). First, the real part of (64)- $(65)$ gives the invariant $E^{a b} E_{a b}-$ $H^{a b} H_{a b}=\Re(I)=\frac{1}{8} C^{a b c d} C_{a b c d}$, and combined with 73 implies

$$
E^{a b} E_{a b}=2 \rho_{\mathrm{g}}+\frac{1}{2} \Re(I), \quad H^{a b} H_{a b}=2 \rho_{\mathrm{g}}-\frac{1}{2} \Re(I) .
$$

Hence, defining the invariants

$$
N_{E} \equiv 2 \xi+\frac{1}{2} \Re(I), \quad N_{H} \equiv 2 \xi-\frac{1}{2} \Re(I)
$$

we obtain the following, new characterizations of a Weyl principal observer:

(v-a) $E^{a b} E_{a b}$ attains minimum value, namely $N_{E}$;

(v-b) $H^{a b} H_{a b}$ attains minimum value, namely $N_{H}$.

Second, the (completely symmetric, spatial) tensors

$$
t_{a b} \equiv h_{a}{ }^{c} h_{b}{ }^{d} T_{c d e f} u^{e} u^{f}, \quad \mathcal{Q}_{a b c} \equiv-h_{a}{ }^{c} h_{b}{ }^{d} h_{c}{ }^{e} T_{c d e f} u^{f}, \quad t_{a b c d} \equiv h_{a}{ }^{c} h_{b}{ }^{d} h_{c}{ }^{e} h_{d}{ }^{f} T_{\text {cdef }}
$$

also appear in the orthogonal splitting of $T_{a b c d}$ relative to $u^{a}, T_{a b c d}=\rho_{\mathrm{g}} u_{a} u_{b} u_{c} u_{d}+4 \mathcal{P}_{(a} u_{b} u_{c} u_{d)}+$ $6 t_{(a b} u_{c} u_{d)}+4 \mathcal{Q}_{(a b c} u_{d)}+t_{a b c d}$; they satisfy $t^{a}{ }_{a}=\rho_{\mathrm{g}}, \mathcal{Q}^{a b}{ }_{b}=\mathcal{P}^{a}$ and $t^{c}{ }_{c a b}=t_{a b}$, can be expressed in terms of $E_{a b}, H_{a b}$, and have been proven important in several contexts [6, 40]. In [39] it was shown that

$$
\begin{aligned}
& t^{a b} t_{a b}-\left(\frac{1}{6} \alpha^{2}-\frac{1}{3} \xi^{2}\right)=\frac{1}{3}\left(\rho_{\mathrm{g}}^{2}-\xi^{2}\right)+\frac{2}{3} \mathcal{P}^{a} \mathcal{P}_{a}, \\
& \mathcal{Q}^{a b c} \mathcal{Q}_{a b c}-\left(2 \xi^{2}-\frac{1}{2} \alpha^{2}\right)=\rho_{\mathrm{g}}^{2}-\xi^{2}+\left(\rho_{\mathrm{g}}^{2}-\mathcal{P}^{a} \mathcal{P}_{a}-\xi^{2}\right), \\
& t^{a b c d} t_{a b c d}-\left(\alpha^{2}+5 \xi^{2}\right)=\rho_{\mathrm{g}}^{2}-\xi^{2}+4\left(\rho_{\mathrm{g}}^{2}-\mathcal{P}^{a} \mathcal{P}_{a}-\xi^{2}\right)
\end{aligned}
$$

are functions $U^{+} \rightarrow \mathbb{R}_{\geq 0}$ with infimum 0 , where by $(75)$ the respective right hand sides are the sums of two such functions. It follows that the functions attain minimum value 0 precisely when $\rho_{\mathrm{g}}=\xi, \mathcal{P}^{a}=0$, such that a Weyl principal observer can be also characterized by either one of the following properties:

(vi-a) $t^{a b} t_{a b}$ takes minimum value, namely $\frac{1}{3} \xi^{2}+\frac{1}{6} \alpha^{2}=\frac{1}{4}\left(\alpha^{2}+q^{2}\right)$;

(vi-b) $\mathcal{Q}^{a b c} \mathcal{Q}_{a b c}$ takes minimum value, namely $2 \xi^{2}-\frac{1}{2} \alpha^{2}=\frac{3}{2} q^{2}$;

(vi-c) $t^{a b c d} t_{a b c d}$ takes minimum value, namely $5 \xi^{2}+\alpha^{2}=\left(9 \alpha^{2}+15 q^{2}\right) / 4$.

By characterization (ii) the existence of a principal observer requires diagonalizability of $\mathrm{C}$ and thus excludes the 'non-diagonal' Petrov types II, III and N. If the Petrov type is $\mathrm{O}$ at $p\left(C_{a b c d}=0\right)$ then every observer is principal, trivially. Hence the non-trivial 'diagonal' Petrov types D and I remain. Since $\mathrm{C}$ is self-adjoint due to $\mathcal{C}_{a b c d}=\mathcal{C}_{c d a b}$ and thus eigenbivectors corresponding to different eigenvalues are orthogonal, there exists an oriented orthonormal frame $\left(\mathcal{X}_{a b}^{i}\right)$ of $S^{+}$such that (cf. [19]):

$$
\mathcal{C}_{a b c d}=\sum_{i=1}^{3} \lambda_{i} \mathcal{X}_{a b}^{i} \mathcal{X}_{c d}^{i}, \quad \mathcal{X}_{a b}^{i}=\left[2\left(e_{0}\right)_{[a}\left(e_{i}\right)_{b]}\right]^{\dagger}=2\left(e_{0}\right)_{[a}\left(e_{i}\right)_{b]}+i 2\left(e_{j}\right)_{[a}\left(e_{k}\right)_{b]} .
$$

Here $\mathcal{X}_{a b}^{i}$ is a unitary self-dual eigenbivector of $\mathrm{C}$ with eigenvalue $\lambda_{i}$; the restricted orthonormal tetrad $\left(e_{0}^{a}, e_{i}^{a}\right)$ realizing (78) is biunivocally related to $\left(\mathcal{X}_{a b}^{i}\right)$, see appendix $\mathrm{A}$, and is referred to as a (Weyl) principal tetrad. Contracting (78) twice with an arbitrary observer $u^{a}$ we obtain

$$
Q^{a b}=\sum_{i=1}^{3} \lambda_{i} x_{i}^{a} x_{i}^{b} \quad \Leftrightarrow \quad \mathrm{Q}=\sum_{i=1}^{3} \lambda_{i} x_{i} x_{i}, \quad x_{i}^{a}=\left(\mathcal{X}^{i}\right)^{a b} u_{b} \equiv \mathfrak{e}_{i}^{a}-i \mathfrak{b}_{i}^{a} .
$$

\footnotetext{
${ }^{9}$ Although implicit in 39] it was not emphasized there that the infimum values for the functions ('super-energy scalars') appearing in characterizations (iv) and (vi) (see below) are acquired precisely by principal observers.
} 
The vectors $x_{i}^{a}$ are eigenvectors of $\mathrm{Q}$ with eigenvalue $\lambda_{i}$, are complex in general, and by (13) form an oriented orthonormal frame of $\mathbb{C} u^{\perp}$ :

$$
x_{m}^{a}\left(x_{n}\right)_{a}=\mathfrak{e}_{m}^{a}\left(\mathfrak{e}_{n}\right)_{a}-\mathfrak{b}_{m}^{a}\left(\mathfrak{b}_{n}\right)_{a}=\delta_{m n}, \quad \mathfrak{e}_{m}^{a}\left(\mathfrak{b}_{n}\right)_{a}+\mathfrak{b}_{m}^{a}\left(\mathfrak{e}_{n}\right)_{a}=0, \quad x_{i}^{a}=\left[x_{j}, x_{k}\right]^{a} .
$$

As a consequence one has, for any $y \in \mathbb{C} u^{\perp}$ :

$$
\left[x_{i}, y\right]^{a}=\left(x_{j}^{b} y_{b}\right) x_{k}^{a}-\left(x_{k}^{b} y_{b}\right) x_{j}^{a} .
$$

Comparison of characterization (ii) of principal observers with 79 tells that the principal observers are precisely those $u^{a}$ for which the vectors $x_{i}^{a}$ can be taken to be real $\left(\mathfrak{b}_{i}^{a}=0 \Leftrightarrow x_{i}^{a}=\mathfrak{e}_{i}^{a}\right)$, which by (19) happens precisely when $u^{a}$ belongs to the blades $\Sigma_{i} \equiv\left\langle e_{0}^{a}, e_{i}^{a}\right\rangle$ of $X_{a b}^{i}$, i.e., equals the first vector $e_{0}^{a}$ of a principal tetrad $\left(e_{0}^{a}, e_{i}^{a}\right)$ used in (78). Hence, principal observers do exist, but principal tetrads and thus principal observers may not be unique; Petrov types D and I provide the relevant distinction:

- Petrov type D, characterized by one repeated and one simple eigenvalue; e.g.

$$
\lambda_{2}=\lambda_{3}=-\lambda_{1} / 2 \equiv \lambda \quad \Rightarrow \quad q=0, \quad \xi=\frac{3}{2}|\lambda|^{2}=\frac{1}{4}|I| .
$$

The endomorphism C (Q) has an up to reflection unique unitary eigenbivector $\mathcal{X}_{a b}^{1} \equiv \mathcal{X}_{a b} \equiv X_{a b}^{\dagger}$ (unit eigenvector $x_{1}^{a} \equiv x^{a}$ ) corresponding to the non-degenerate eigenvalue $\lambda_{1}=-2 \lambda$; the blade $\Sigma_{1} \equiv \Sigma$ of $X_{a b}$ is thus uniquely defined and called the (Weyl) timelike principal plane, and its two null directions the (Weyl) principal null directions (PNDs), spanned by null vectors $k^{a}$ that satisfy $C_{a b c[d} k_{f]} k^{b} k^{c}=0[19,41]$. The pair $\left(\mathcal{X}_{a b}^{2}, \mathcal{X}_{a b}^{3}\right)$ is only determined up to a complex rotation, and so the same holds for $\left(x_{2}^{a}, x_{3}^{a}\right)$ in 79 . However, we can rewrite 79 as

$$
Q^{a}{ }_{b}=\lambda\left(h_{b}^{a}-3 x^{a} x_{b}\right) \quad \Leftrightarrow \quad \mathrm{Q}=\lambda(\mathrm{h}-3 \times \mathrm{xx}), \quad x^{a} \equiv \mathfrak{e}^{a}-i \mathfrak{b}^{a} .
$$

Hence characterization (ii) of principal observers is equivalent to

(ii)' the non-degenerate unit eigenvector $x^{a}$ is real $\left(\mathfrak{b}^{a}=0 \Leftrightarrow x^{a}=\mathfrak{e}^{a}\right)$, i.e., $u^{a}$ belongs to $\Sigma$. The principal tetrads are $\left(E_{0}^{a}, \pm E_{1}^{a}, E_{2}^{a}, \pm E_{3}^{a}\right)$ with $\left(E_{0}^{a}, E_{i}^{a}\right)$ as in (32)-(33) [42].

- Petrov type I, characterized by all $\lambda_{i}$ 's being distinct $\left(\lambda_{i} \neq \lambda_{j}\right.$ for $\left.i \neq j\right)$. Once the eigenvalues $\lambda_{i}$ have been put in a certain order the oriented frame $\left(\mathcal{X}_{a b}^{i}\right)$ in $(78)$ is determined up to a simultaneous reflection of two elements, and so the same holds for the triad $\left(e_{i}^{a}\right)$; hence there are 24 principal tetrads, but we will speak about 'the (essentially unique) principal tetrad' $\left(e_{0}^{a}, e_{i}^{a}\right)$. There are four simple Weyl PNDs, spanned by vectors $k^{a}$ that satisfy $C_{a b c[d} k_{f]} k^{b} k^{c}=0 \neq C_{a b c[d} k_{f]} k^{c}$ [19, 41]. Characterization (ii) of principal observers becomes:

(ii)' the unit eigenvectors $x_{i}^{a}$ of $\mathrm{Q}$ are real $\left(x_{i}^{a}=\mathfrak{e}_{i}^{a}=e_{i}^{a}\right)$, i.e., $u^{a}$ is the unique observer $e_{0}^{a}$ lying along the joint intersection of the three timelike blades $\Sigma_{i}$.

Remark 5.2. In the gravitational case the existence of Weyl principal observers is equivalent to diagonalizability of the endomorphism $\mathrm{C}$ (or of the endomorphism $T^{a b}{ }_{c d}$ on the space of traceless symmetric tensors, see [38, 43] and appendix B.2). In fact this parallels the EM case: by (10)-(11) and (23) one has $\mathcal{F}^{a}{ }_{b} \mathcal{F}^{b}{ }_{c}=I_{F} \delta_{c}^{a}$ and $T^{a}{ }_{b} T^{b}{ }_{c}=\chi^{2} \delta_{c}^{a}$. This implies that the endomorphism $\mathcal{F}^{a}{ }_{b}$ of $\mathbb{C} T_{p} M$ is nilpotent and thus non-diagonalizable in the non-trivial null case $F_{a b} \neq 0, I_{F}=0$, and diagonalizable in the other two cases $F_{a b}=0$ and $I_{F} \neq 0$. Hence diagonalizability of $\mathcal{F}_{b}^{a}$ is equivalent to the existence of EM principal observers, and the same statement holds when $\mathcal{F}^{a}{ }_{b}$ is replaced by $F_{b}^{a}$ (cf. p. 182 of [30]) or $T^{a}{ }_{b}$. Contrary to the EM case, diagonalizability of $\mathrm{C} \neq 0$, or equivalently of $\mathrm{Q} \neq 0$ for any observer $u^{a}$, cannot be expressed entirely in terms of the invariants $I$ and $J$, since $I^{3}=6 J^{2} \neq 0$ does not distinguish Petrov type II from D; however, $I^{3} \neq 6 J^{2}$ distinguishes Petrov type I, which is generic and diagonal, while $I=J=0, C \neq 0$ corresponds to the non-diagonal Petrov types III and N. 
Remark 5.3. Consider the condition $q=0$. It is automatically satisfied in the Petrov type $\mathrm{D}$ case, see (82), while for Petrov type I precisely gives the subcase where the invariant $M \equiv I^{3} / J^{2}-6$ is real positive or infinite $(J=0)$, or equivalently where the four Weyl PNDs span a $3 \mathrm{~d}$ (instead of a $4 \mathrm{~d}$ ) vector space; see appendix B.2. In general, one has $q=\Im\left(\lambda_{j} \bar{\lambda}_{k}\right)=\Im\left(\lambda_{j} / \lambda_{k}\right)$ for any $\lambda_{k} \neq 0$, such that $q=0$ corresponds to the ratio of any two non-zero eigenvalues being real, i.e., there exist real numbers $\lambda_{k}^{\prime}$ summing to zero such that $\lambda_{k}=e^{i \sigma} \lambda_{k}^{\prime}=\cos (\sigma) \lambda_{k}^{\prime}+i \sin (\sigma) \lambda_{k}^{\prime}, k=1,2,3$, where $\sigma \in[0, \pi[$ is a duality rotation index (see 44. for further discussions, and cf. remark 4.6). Then, characterizations (ii) and (vi-b) of Weyl principal observers become equivalent to [6]:

(ii) $^{*} E_{a b}$ and $H_{a b}$ are linearly dependent, $\cos (\sigma) H_{a b}+\sin (\sigma) E_{a b}=0$;

$\left(\right.$ vi-b) ${ }^{*} \mathcal{Q}_{a b c}=0$.

Here (ii)* is a substitute for characterization (i) of Weyl principal observers, which resembles characterization (i) of EM principal observers even more than the general commutation condition; because of $\mathcal{P}^{a}=Q^{a b}{ }_{b}$ characterization (vi-b)* may be seen as a stricter form of the definition $\mathcal{P}^{a}=0$ of Weyl principal observers. Regarding characterizations (iv) and (v), by 770-771, (77) and 22 applied to $z=I$ the minimum values for $\rho_{\mathrm{g}}$ and $\sqrt{E^{a b} E_{a b}}, \sqrt{H^{a b} H_{a b}}$ respectively become $\xi=|I| / 4$ and $\sqrt{N_{E}}=|\Re(I)|, \sqrt{N_{H}}=|\Im(I)|$, which formally match the corresponding values (27) and (30) of the EM case. Spacetimes of Petrov type D or of Petrov type I with $q=0$ at each point have been called super-energy non-radiative gravitational fields [6, 13]. In general one has $I /|I|=e^{2 i \sigma}$. The subcase $\sigma=0$ $(\sigma=\pi / 2)$ corresponds to purely electric (purely magnetic) spacetimes, which by (64) are characterized by all eigenvalues $\lambda_{k}$ being real (purely imaginary), or by $I=|I|>0(I=-|I|<0)$ and $M$ being real non-negative or infinite; by (65) any observer measures $E^{a b} E_{a b}>H^{a b} H_{a b}\left(E^{a b} E_{a b}<H^{a b} H_{a b}\right)$, and the principal observers are precisely those for which $H_{a b}\left(E_{a b}\right)$ vanishes; cf. remark 4.6, and see [45, 37. Large and important classes of purely electric spacetimes exist; for instance all static spacetimes, and all spacetimes which exhibit spherical, hyperbolic, or planar symmetry, which are automatically of Petrov type D (or O) [46, 47, 48,. See [35, 49, 50, 51] for surveys of the literature on purely electric or magnetic spacetimes.

Remark 5.4. In a vacuum spacetime, without sources but with a possible cosmological constant $\Lambda$ $\left(T_{a b}=0, R_{a b}=\Lambda g_{a b}\right)$ the gravitoelectric and gravitomagnetic tidal tensors relative to an observer $u^{a}$ are respectively given by $\left(E_{\mathrm{R}}\right)_{a b} \equiv R_{a c b d} u^{c} u^{d}=E_{a b}-\Lambda / 3 h_{a b}$ and $\left(H_{\mathrm{R}}\right)_{a b} \equiv \star R_{a c b d} u^{c} u^{d}=R_{\star a c b d} u^{c} u^{d}=H_{a b}$, and can be assembled into $\left(Q_{\mathrm{R}}\right)_{a b}=\left(E_{\mathrm{R}}\right)_{a b}-i\left(H_{\mathrm{R}}\right)_{a b}=Q_{a b}-\Lambda / 3 h_{a b}$, where $E_{a b}, H_{a b}, Q_{a b}$ are the relative tensors associated to the Weyl tensor defined above. The endomorphism of $\mathbb{C} u^{\perp}$ associated to $\left(Q_{\mathrm{R}}\right)_{a b}$ has eigenvalues $\lambda_{k, \mathrm{R}}=\lambda_{k}-\Lambda / 3$ and invariants $I_{\mathrm{R}}=I+\Lambda^{2} / 3, J_{\mathrm{R}}=J-\Lambda I-\Lambda^{3} / 9$, defined as in 665), 67). The corresponding super-Poynting vector and super-energy density defined as in (72), (73) are simply given by $\mathcal{P}_{\mathrm{R}}^{a}=\mathcal{P}^{a}$ and $\rho_{\mathrm{g}, \mathrm{R}}=\rho_{\mathrm{g}}+\Lambda^{2} / 12$. For any value of $\Lambda$ it follows that the corresponding Riemann principal observers are the Weyl principal observers, with characterizations analogous to (i)(vi). Also, the spacetime is Riemann purely electric if and only if it is Weyl purely electric, and is never Riemann purely magnetic for $\Lambda \neq 0$ [35, 37].

\subsection{Petrov type D: general vs. principal observers}

The non-null EM and Petrov type D gravitational cases both exhibit a distinguished unitary self-dual bivector $\mathcal{X}_{a b}=X_{a b}-i \star X_{a b}$, where the blade of $X_{a b}$ (viz. the timelike principal plane $\Sigma$ ) contains the principal observers. Concretely, $\mathcal{X}_{a b}=F_{a b}^{\dagger} / \sqrt{I_{F}}$ in the former case, while in the latter case $\mathcal{X}_{a b}$ generates the eigendirection of $\mathrm{C}$ corresponding to the non-degenerate eigenvalue $-2 \lambda$. Given an observer $u^{a}$, the role of $x^{a} \equiv \mathcal{X}^{a b} u_{b} \equiv \mathfrak{e}^{a}-i \mathfrak{b}^{a}$ is played by $\left(E^{a}-i B^{a}\right) / \sqrt{I_{F}}$ and a unit eigenvector of $\mathrm{Q}$ with non-degenerate eigenvalue $-2 \lambda$, respectively.

Here we strengthen the analogy by transferring the EM Wheeler result to the Petrov D gravitational case. Take any observer $u^{a}$, and consider the final formula in 72 for the associated super-Poynting vector. On comparing to (24) there is an important conceptual difference between the definitions of a Poynting and super-Poynting vector: the former is a spatial vector product while the latter is dual to a commutator of endomorphisms corresponding to spatial 2-tensors. For Petrov type D, however, we have 
the special form 83 and its complex conjugate for the relevant endomorphisms $Q$ and $\bar{Q}$; since the identity map $\mathrm{h}$ of $\mathbb{C} u^{\perp}$ commutes with any other endomorphism of $\mathbb{C} u^{\perp}$, the dual to the commutator of $\mathrm{Q}$ and $\overline{\mathrm{Q}}$ is parallel to the spatial vector product of the respective unit non-degenerate eigenvectors $x^{a}$ and $\bar{x}^{a}$ by the crucial identity (5). Using also 822 and $[x, \bar{x}]^{a}=2 i[\mathfrak{e}, \mathfrak{b}]^{a}$ we obtain

$$
\mathcal{P}^{a}=\frac{1}{4 i}[\mathrm{Q}, \overline{\mathrm{Q}}]^{a}=\frac{1}{4 i}[\lambda(\mathrm{h}-3 \mathrm{xx}), \bar{\lambda}(\mathrm{h}-3 \overline{\mathrm{xx}})]^{a}=\frac{9|\lambda|^{2}}{4 i} x^{b} \bar{x}_{b}[x, \bar{x}]^{a}=3 \xi x^{b} \bar{x}_{b}[\mathfrak{e}, \mathfrak{b}]^{a} .
$$

As in the non-null EM case, we conclude by lemma 4.2 that the plane $\left\langle u^{a}, \mathcal{P}^{a}\right\rangle$ intersects $\Sigma$ in $\left\langle u_{\Sigma}^{a}\right\rangle$, where $u_{\Sigma}^{a}$ is the principal observer along the projection of $u^{a}$ onto $\Sigma$; moreover, we have by (37)-38):

$$
\begin{aligned}
\mathcal{P}^{a} & =3 \xi \cosh \left(2 \psi_{\Sigma}\right) \cosh ^{2}\left(\psi_{\Sigma}\right) v\left(u_{\Sigma}, u\right)^{a}=\frac{3 \xi}{4} \sinh \left(4 \psi_{\Sigma}\right) \widehat{v}\left(u_{\Sigma}, u\right)^{a} \\
& =-\frac{3 \xi}{4} \sinh \left(4 \psi_{\Sigma}\right)\left[\sinh \left(\psi_{\Sigma}\right) u_{\Sigma}^{a}+\cosh \left(\psi_{\Sigma}\right) u_{\perp}^{a}\right] .
\end{aligned}
$$

Notice the resemblance with 45). Analogously, the final formula in 73 and $\operatorname{tr}(\mathrm{h})=3, \operatorname{tr}(\mathrm{xx})=\operatorname{tr}(\overline{\mathrm{xx}})=$ $1, \operatorname{tr}(\mathrm{xx} \overline{\mathrm{xx}})=\left(x^{a} \bar{x}_{a}\right)^{2}$ produce

$$
\rho_{\mathrm{g}}=\frac{1}{4} \operatorname{tr}(\mathrm{Q} \overline{\mathrm{Q}})=\frac{3|\lambda|^{2}}{4}\left[3\left(x^{a} \bar{x}_{a}\right)^{2}-1\right]=\xi+\frac{3 \xi}{2} \sinh ^{2}\left(2 \psi_{\Sigma}\right)=\frac{\xi}{4}+\frac{3 \xi}{4} \cosh \left(4 \psi_{\Sigma}\right) .
$$

Note that $\rho_{\mathrm{g}} \geq \xi$ and $\rho_{\mathrm{g}}=\xi \Leftrightarrow \psi_{\Sigma}=0 \Leftrightarrow \mathcal{P}^{a}=0$, cf. 75 and characterization (iv) of Weyl principal observers. Comparing to 85 , we arrive at our main

Theorem 5.5 (Gravitational Wheeler analogue for Petrov type D). Suppose the Weyl tensor is of Petrov type $D$ at a point $p$, and let $u^{a}$ be a non-principal observer measuring a super-Poynting vector $\mathcal{P}^{a}$ and super-energy density $\rho_{g}$ at $p$. Then there is a principal observer instantaneously traveling relative to $u^{a}$ in the direction of $\mathcal{P}^{a}$, namely the observer $u_{\Sigma}^{a}$ lying along the orthogonal projection of $u^{a}$ onto the Weyl principal plane $\Sigma$, with spatial velocity

$$
v\left(u_{\Sigma}, u\right)^{a}=v_{\| \mathcal{P}}^{a} \equiv \frac{2 \mathcal{P}^{a}}{3 \xi \zeta_{g}\left(\zeta_{g}+1\right)}, \quad \zeta_{g}=\sqrt{1+\frac{2\left(\rho_{g}-\xi\right)}{3 \xi}}=\cosh \left(2 \psi_{\Sigma}\right) .
$$

Hence

$$
u_{\Sigma}^{a}=\cosh \left(\psi_{\Sigma}\right)\left[u^{a}+v\left(u_{\Sigma}, u\right)^{a}\right], \quad \cosh \left(\psi_{\Sigma}\right)=\sqrt{\frac{\zeta_{g}+1}{2}} .
$$

Remark 5.6. Remark 4.5 has a clear analogue in the present context. By 86 a non-zero super-Poynting vector corresponds to a unique non-principal observer $u^{a}$; in particular,

$$
\sqrt{\mathcal{P}^{a} \mathcal{P}_{a}}=\frac{3 \xi}{4} \sinh \left(4 \psi_{\Sigma}\right)=3 \xi\left[2 \cosh ^{2}\left(\psi_{\Sigma}\right)-1\right] \cosh \left(\psi_{\Sigma}\right) \sqrt{\cosh ^{2}\left(\psi_{\Sigma}\right)-1},
$$

and in conjunction with (87) this gives the following analogue of the Wheeler equation (1):

$$
\tanh \left(4 \psi_{\Sigma}\right)=\sqrt{\mathcal{P}^{a} \mathcal{P}_{a}} /\left(\rho_{\mathrm{g}}-\xi / 4\right) .
$$

This expresses the ratio of two functions of the single variable $4 \psi_{\Sigma}$ increasing quartically (instead of quadratically in the non-null EM case) with the relative Lorentz factor $\cosh \left(\psi_{\Sigma}\right)$ of $u^{a}$ and $u_{\Sigma}^{a}$; also $E^{a b} E_{a b}-N_{E}^{2}=H^{a b} H_{a b}-N_{H}^{2}=3 \xi \sinh ^{2}\left(2 \psi_{\Sigma}\right)$, with the same behaviour. Equation (52) remains formally the same and the corresponding observer geometry is analogous. $U^{+}$is foliated by $2 \mathrm{~d}$ leafs $\mathcal{L}(\gamma)$, consisting of the observers $u^{a}$ with $u_{\Sigma}^{a}=E_{0}^{a}(\gamma)$, and for given $u^{a} \in \mathcal{L}(\gamma)$ the vector $\mathfrak{e}^{a} \propto \mathfrak{e}_{\Sigma}^{a}$ is now an eigenvector of the endomorphism $\mathrm{Q}_{\Sigma} \equiv \mathrm{E}_{\Sigma}-i \mathrm{H}_{\Sigma}$ associated to $u_{\Sigma}^{a}$ with simple eigenvalue $-2 \lambda$, and thus of $\mathrm{E}_{\Sigma}$ and $\mathrm{H}_{\Sigma}$. Comments on the 2-plane $\left\langle u^{a}, \mathcal{P}^{a}\right\rangle$ and the 2d level surface of observers with the same energy density $\rho_{\mathrm{g}}=\rho_{\mathrm{g}}\left(\psi_{\Sigma}\right)$ (or norm of the super-Poynting vector) are analogous, mutatis mutandis. 


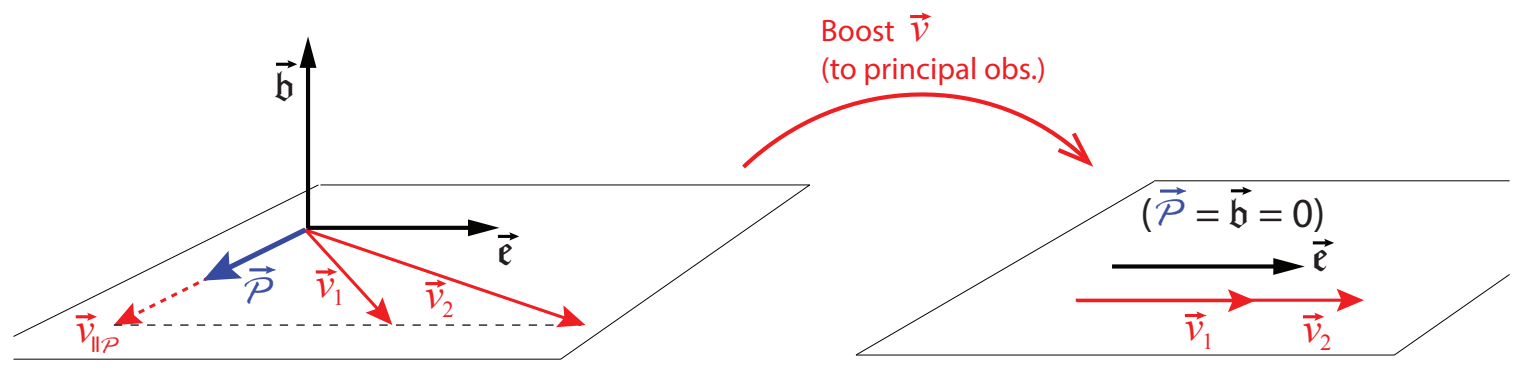

Figure 2: Velocities of the Weyl principal observers, represented in the rest space of a generic observer observer $u^{a}$ (measuring $\mathfrak{e}^{a}, \mathfrak{b}^{a}, \mathrm{E}, \mathrm{H}$, and a super-Poynting vector $\mathcal{P}^{a}$ ). They move with a relative velocity $v^{a}$ that has a fixed component $v_{\| \mathcal{P}}^{a}$ along $\mathcal{P}^{a}$, given in (88), and a 'free' component $v_{\| \mathfrak{e}}^{a}$ along $\mathfrak{e}^{a}$. When $u^{a}$ is principal (right panel), the non-degenerate eigendirection of $\mathrm{E}$ and $\mathrm{H}$ is $\left\langle\mathfrak{e}^{a}\right\rangle$, and all other principal observers are obtained by boosting along this direction with arbitrary rapidity.

Remark 5.7. When $u^{a}=u_{\Sigma}^{a}$ is principal the associated endomorphism $Q_{\Sigma}$ admits three real orthogonal eigendirections [see characterization (ii) of Weyl principal observers] which are common eigendirections of $\mathrm{E}_{\Sigma}$ and $\mathrm{H}_{\Sigma}$. When $u^{a}$ is non-principal, it follows from (83), (84) and $x_{a}[x, \bar{x}]^{a}=0$ that $\mathcal{P}^{a} \neq 0$ is an eigenvector of the associated endomorphism $\mathrm{Q}=\mathrm{E}-i \mathrm{H}$ with degenerate eigenvalue $\lambda$, and thus of $\mathrm{E}$ and $\mathrm{H}$ with respective eigenvalues $\Re(\lambda)$ and $-\Im(\lambda)$; moreover, the real eigenvectors of $\mathrm{Q}$ are precisely the common (real) eigenvectors of $\mathrm{E}$ and $\mathrm{H}$; if there were two independent such eigenvectors then, since $Q_{a b}$ and $E_{a b}, H_{a b}$ are symmetric, their vector product would give a third real eigenvector and $u^{a}$ would be principal by characterization (ii) of Weyl principal observers, a contradiction; hence, when $u^{a}$ is non-principal, $\left\langle\mathcal{P}^{a}\right\rangle$ is the only real eigendirection of $\mathrm{Q}$ and the only common eigendirection of $\mathrm{E}$ and $\mathrm{H}$.

Similarly to (57), the velocities of the principal observers $u^{\prime a} \in \Sigma$ relative to an arbitrary observer $u^{a}$ are the orthogonal sum of a fixed component along $\mathcal{P}^{a}$ and a 'free' component along $\left\langle\mathfrak{e}^{a}\right\rangle$ :

$$
v\left(u^{\prime}, u\right)^{a}=v_{\| \mathcal{P}}^{a}+v_{\| \mathfrak{e}}^{a}, \quad v_{\| \mathfrak{e}}^{a}=\tanh (\varphi) \frac{2 \mathfrak{e}^{a}}{\zeta_{\mathrm{g}}+1}, \varphi \in \mathbb{R}=\text { 'free' vector along }\left\langle\mathfrak{e}^{a}\right\rangle,
$$

with associated Lorentz factor $\cosh (\varphi) \cosh \left(\psi_{\Sigma}\right)=\cosh (\varphi) \sqrt{\left(\zeta_{\mathrm{g}}+1\right) / 2}$. This is illustrated in Fig. 2 notice the resemblance with the EM diagram in Fig. 1. To calculate $\mathfrak{e}^{a}$ directly from the electric and magnetic parts of the Weyl tensor as measured by $u^{a}$, one has to construct a complex eigenvector $w^{a}$ of $\mathrm{Q}$ with eigenvalue $-2 \lambda=2 J / I$, normalize it to a unit vector $x^{a}$, and take the real part. To construct $w^{a}$ one can apply the standard technique of solving the linear system of equations $\left(Q_{m^{\prime} i^{\prime}}+2 \lambda \delta_{m^{\prime} i^{\prime}}\right) w^{i^{\prime}}=0$, where $\left(e_{i^{\prime}}^{a}\right)$ is an arbitrary orthonormal triad of $u^{\perp}$ and $w^{a}=w^{i^{\prime}} e_{i^{\prime}}^{a}$. Alternatively, one can take any $y^{a} \in u^{\perp}$ that is not an eigenvector of $\mathbf{Q}$ with eigenvalue $\lambda$ (i.e., any real vector not orthogonal to $\mathfrak{e}^{a}=\mathfrak{e}_{\Sigma}^{a}$ when $u^{a}=u_{\Sigma}^{a}$ is principal, and any real vector not proportional to $\mathcal{P}^{a}$ when $u^{a}$ is non-principal, see remark 5.7. where for an orthonormal triad of $u^{\perp}$ at least one of the vectors $e_{i^{\prime}}^{a}$ may be taken as $y^{a}$ ) and construct $w^{a}$ from

$$
w^{a}=\left(Q_{b}^{a}-\lambda h_{b}^{a}\right) y^{b} \quad\left[w^{a}=\left(Q_{i^{\prime}}^{m^{\prime}}-\lambda \delta_{i^{\prime}}^{m^{\prime}}\right) e_{m^{\prime}}^{a}\right],
$$

where the result for $y^{a}=e_{i^{\prime}}^{a}$ is indicated between square brackets. Applying (2) to $z=w^{b} w_{b}$ we obtain

$$
\mathfrak{e}^{a}=\Re\left(\frac{w^{a}}{\sqrt{z}}\right)=\sqrt{\frac{|z|+\Re(z)}{2|z|^{2}}} \Re\left(w^{a}\right)+\operatorname{sgn}(\Im(z)) \sqrt{\frac{|z|-\Re(z)}{2|z|^{2}}} \Im\left(w^{a}\right), \quad z \equiv w^{b} w_{b} .
$$

Thus, an observer $u^{a}$ passing through a point $p$ and measuring $Q^{a b}=\left(E^{m^{\prime} n^{\prime}}-i H^{m^{\prime} n^{\prime}}\right) e_{m^{\prime}}^{a} e_{n^{\prime}}^{b}$ may check whether the gravitational field is of Petrov type $\mathrm{D}$ at $p$, and in this case determine all principal observers directly from $Q^{a b}$, by the following 
Algorithm for obtaining the principal observers of a Petrov type D Weyl tensor, directly from electric and magnetic parts of the Weyl tensor measured by an arbitrary observer:

1. Compute $I$ and $J$ from 65) and (67); if $I \neq 0$ and $\left(Q^{a}{ }_{c}-\lambda h^{a}{ }_{c}\right)\left(Q^{c}{ }_{b}+2 \lambda h^{c}{ }_{b}\right)=0, \lambda \equiv-J / I$ the gravitational field is of Petrov type D at $p$ (see appendix B.1), and then go to step 2.

2. Compute $\mathcal{P}^{a}, \rho_{\mathrm{g}}, \xi$ from $(72),(73),(82)$; then 88 gives the velocity relative to $u^{a}$ of the projected principal observer $u_{\Sigma}^{a}$ realizing the gravitational Wheeler analogue.

3. Compute $\mathfrak{e}^{a}$ from $(93)-(94)$; then $(88)$ and $(92)$ give the relative velocities of all principal observers $u^{\prime a}$.

\subsubsection{Doubly aligned non-null Einstein-Maxwell fields}

Gravitational fields that have an energy-momentum tensor of the form (51) where $\mathcal{F}_{a b}=F_{a b}^{\dagger}$ is non-null and satisfies Maxwell's equations $\nabla^{b} \mathcal{F}_{a b}=0$ are said to be of non-null Einstein-Maxwell type; such fields thus exhibit an EM principal plane $\Sigma_{F}$ and a pair of EM PNDs at each point. Wherever the Weyl tensor $C_{a b c d}$ is moreover of Petrov type D there is also a Weyl principal plane $\Sigma_{C}$ and a pair of Weyl PNDs.

Generally one has $\Sigma_{F} \neq \Sigma_{C}$, meaning that the pairs of EM and Weyl PNDs do not coincide and, for a given observer $u^{a}$, the projected observers $u_{\Sigma_{F}}^{a}$ and $u_{\Sigma_{C}}^{a}$ are distinct. Let $\mathcal{X}_{a b}^{F}$ and $\mathcal{X}_{a b}^{C}$ denote unitary self-dual bivectors, defined up to sign by (31) and as the unitary eigenbivector of $\mathrm{C}$ with non-degenerate eigenvalue $-2 \lambda=2 J / I$, respectively. Since $\Sigma_{F}$ and $\Sigma_{C}$ are the blades of their real parts, and since these real parts are bijectively related to the bivectors themselves by (8), one has $\Sigma_{F}=\Sigma_{C}$ precisely when $\mathcal{X}_{a b}^{F}= \pm \mathcal{X}_{a b}^{C}$, which by $(31)$ happens precisely when $\mathcal{F}_{a b}$ is an eigenbivector of $\mathrm{C}$ with non-degenerate eigenvalue $-2 \lambda, \mathrm{C}(\mathcal{F})_{a b}=-2 \lambda \mathcal{F}_{a b}$. This can be verified directly by any observer $u^{a}$, since by (34) and 63 this comes down to $f^{a}$ being an eigenvector of $\mathrm{Q}$ with eigenvalue $-2 \lambda$ :

$$
\Sigma_{F}=\Sigma_{C} \quad \Leftrightarrow \quad Q_{b}^{a} f^{b}=-2 \lambda f^{a}, \quad f^{a}=E^{a}-i B^{a} .
$$

If this holds everywhere in the considered spacetime region (with the possible exception of a measure zero set of points where $C_{a b c d}=0 \Leftrightarrow \lambda=0$ ) one speaks about Petrov type $\mathrm{D}$ doubly aligned non-null Einstein-Maxwell fields. At the points where $C_{a b c d} \neq 0$ the pairs of EM and Weyl PNDs coincide, and the principal observers and tetrads of the EM and gravitational fields are the same. For any observer $u^{a}$ we have $u_{\Sigma_{F}}^{a}=u_{\Sigma_{C}}^{a} \equiv u_{\Sigma}^{a}$, and thus $\psi_{\Sigma_{F}}=\psi_{\Sigma_{C}} \equiv \psi_{\Sigma}$ and $\zeta_{\mathrm{em}}=\zeta_{\mathrm{g}}$ in 49 ) and (88), and also $\mathfrak{e}_{F}^{a}=\mathfrak{e}_{C}^{a}=\mathfrak{e}^{a}$ in (58) and (94). It follows that the relative super-energy density is a quadratic function of the energy density, and the super-Poynting vector is aligned with the Poynting vector:

$$
\rho_{\mathrm{g}}=\frac{\xi}{2}\left[3\left(\frac{\rho_{\mathrm{em}}}{\chi}\right)^{2}-1\right], \quad \mathcal{P}^{a}=\frac{3 \xi}{2 \chi} \frac{\rho_{\mathrm{em}}}{\chi} p^{a} .
$$

All Petrov type D doubly aligned non-null Einstein-Maxwell fields (with a possible non-zero cosmological constant, and including the vacuum limits obtained by putting the EM parameters to zero) are known; the line elements are exhausted by those constructed in Refs. [21, comprising all well-known black hole solutions such as the Schwarzschild-Kottler, Reissner-Nordström, Kerr-Newman, (charged and/or spinning) $C$ and Plebański-Demiański metrics. We will treat the Kerr-Newman spacetimes in Sec. 6.2 .

\subsection{Petrov type I: general vs. principal observers}

In the Petrov type I case the three eigenvalues $\lambda_{i}$ of $\mathrm{C}$ (equal to those for any $\mathrm{Q}$ ) are distinct. There is a unique principal observer $e_{0}^{a}$ and an essentially unique principal tetrad $\left(e_{0}^{a}, e_{i}^{a}\right)$, where the spacelike vectors $e_{i}^{a} \in e_{0}^{\perp}$ are unit eigenvectors of the associated endomorphism $\mathrm{Q}_{0}=\mathrm{E}_{0}-i \mathrm{H}_{0}$ of $e_{0}^{\perp}$. The unitary self-dual eigenbivectors $\mathcal{X}_{a b}^{i}$ of $\mathrm{C}$ are related to the principal tetrad vectors by 78 .

Consider an arbitrary observer $u^{a} \in U^{+}$. Its expansion in the principal tetrad $\left(e_{0}^{a}, e_{i}^{a}\right)$ is given by

$$
u^{a}=u^{0} e_{0}^{a}+u^{i} e_{i}^{a}, \quad u^{0}=\sqrt{1+\left(u^{1}\right)^{2}+\left(u^{2}\right)^{2}+\left(u^{3}\right)^{2}},
$$


where the components $u^{i} \in \mathbb{R}$ play the role of independent parameters. Equation (97) defines the boost from $e_{0}^{a}$ to $u^{a}$, with $\cosh \psi\left(u, e_{0}\right)=\cosh \psi\left(e_{0}, u\right)=u^{0}$ and $v\left(u, e_{0}\right)^{a}=u^{i} e_{i}^{a} / u^{0}$. The relative velocity

$$
v\left(e_{0}, u\right)^{a}=e_{0}^{a} / u^{0}-u^{a}
$$

describes the inverse boost. We are interested in an algorithm that derives $v\left(e_{0}, u\right)^{a}$ directly from the tensor $Q_{a b}=E_{a b}-H_{a b}$ relative to $u^{a}$, the dependence of the super-energy-density $\rho_{\mathrm{g}}$ on the parameters $u^{i}$, and to what extent a surrogate of theorem 5.5 holds.

To deal with the first point we look at the eigenvectors $x_{i}^{a}=\mathfrak{e}_{i}^{a}-i \mathfrak{b}_{i}^{a}$ of Q. By (79) these are simply the contractions of the eigenbivectors $\mathcal{X}_{a b}^{i}$ of $\mathrm{C}$ with $u^{a}$, and by (78) and (97) we obtain their expansions in the principal tetrad:

$$
x_{i}^{a}=u^{i} e_{0}^{a}+u^{0} e_{i}^{a}+i\left(u^{k} e_{j}^{a}-u^{j} e_{k}^{a}\right) \quad \Leftrightarrow \quad \mathfrak{e}_{i}^{a}=u^{i} e_{0}^{a}+u^{0} e_{i}^{a}, \quad \mathfrak{b}_{i}^{a}=u^{j} e_{k}^{a}-u^{k} e_{j}^{a} .
$$

In addition to 80 we thus have

$$
\begin{aligned}
& x_{i}^{a}\left(\overline{x_{i}}\right)_{a}=2\left(u^{0}\right)^{2}-2\left(u^{i}\right)^{2}-1=2\left(u^{j}\right)^{2}+2\left(u^{k}\right)^{2}+1, \quad x_{j}^{a}\left(\overline{x_{k}}\right)_{a}=-2 u^{j} u^{k}+2 i u^{0} u^{i}, \\
& \mathfrak{e}_{i}^{a}\left(\mathfrak{e}_{i}\right)_{a}=\left(u^{0}\right)^{2}-\left(u^{i}\right)^{2}=\left(u^{j}\right)^{2}+\left(u^{k}\right)^{2}+1=\mathfrak{b}_{i}^{a}\left(\mathfrak{b}_{i}\right)_{a}+1, \quad \mathfrak{e}_{i}^{a}\left(\mathfrak{b}_{i}\right)_{a}=0, \\
& \mathfrak{e}_{j}^{a}\left(\mathfrak{e}_{k}\right)_{a}=\mathfrak{b}_{j}^{a}\left(\mathfrak{b}_{k}\right)_{a}=-u^{j} u^{k}, \quad \mathfrak{e}_{j}^{a}\left(\mathfrak{b}_{k}\right)_{a}=-\mathfrak{b}_{j}^{a}\left(\mathfrak{e}_{k}\right)_{a}=u^{0} u^{i} .
\end{aligned}
$$

Note that the vectors $\mathfrak{e}_{i}^{a}$ are linearly independent and thus form a (generally non-orthogonal) basis of $u^{\perp}$; moreover one has

$$
u^{0} \mathfrak{b}_{i}^{a}=u^{j} \mathfrak{e}_{k}^{a}-u^{k} \mathfrak{e}_{j}^{a}, \quad u^{i} \mathfrak{b}_{i}^{a}=0,
$$

which gives the expansion of the vectors $\mathfrak{b}_{i}^{a}$ in this basis and shows that they are linearly dependent. The frames $\left(e_{0}^{a}, e_{i}^{a}\right)$ and $\left(u^{a}, x_{i}^{a}\right)$ of $\mathbb{C} T_{p} M$ are both oriented and orthonormal, the expansion of the latter in terms of the former being given by (97) and (99). Inverting this relation and using (103) yields ${ }^{10}$

$$
\begin{aligned}
& e_{0}^{a}=u^{0} u^{a}-u^{i} x_{i}^{a}=u^{0} u^{a}-u^{i} \mathfrak{e}_{i}^{a}, \\
& e_{i}^{a}=-u^{i} u^{a}+u^{0} x_{i}^{a}+i\left(u^{j} x_{k}^{a}-u^{k} x_{j}^{a}\right)=-u^{i} u^{a}+u^{0} \mathfrak{e}_{i}^{a}+u^{j} \mathfrak{b}_{k}^{a}-u^{k} \mathfrak{b}_{j}^{a},
\end{aligned}
$$

which confirms that $e_{0}^{a}$ and $e_{i}^{a}$ are real vectors. From (104) we thus obtain

$$
v\left(e_{0}, u\right)^{a}=-u^{i} x_{i}^{a} / u^{0}=-u^{i} \mathfrak{e}_{i}^{a} / u^{0} .
$$

Alternatively, one can consider the decomposition $z^{a}=z^{0} e_{0}^{a}+\pi(z)^{a}$ of a vector $z^{a} \in T_{p} M$, where

$$
\pi(z)^{a} \equiv\left[\delta_{b}^{a}+e_{0}^{a}\left(e_{0}\right)_{b}\right] z^{b}=z^{i} e_{i}^{a}
$$

is the projection of $z^{a}$ onto the rest space $e_{0}^{\perp}$ of the principal observer. If $z^{a}$ belongs to the rest space $u^{\perp}$ of the given observer (97) then $z^{a} u_{a}=0$, which translates to $u^{0} z^{0}=\sum_{i=1}^{3} u^{i} z^{i}$ and by 99 yields

$$
z^{a}=z^{i} \mathfrak{e}_{i}^{a} / u^{0} .
$$

Applying this to $z^{a}=v\left(e_{0}, u\right)^{a}$ one has $z^{i} e_{i}^{a}=\pi\left(v\left(e_{0}, u\right)\right)^{a}=-\pi(u)^{a}=-u^{i} e_{i}^{a}$ by (98) and (97), which gives $z^{i}=-u^{i}$ and thus (106). Using (100)-(102) we arrive at:

Proposition 5.8. The velocity of $e_{0}^{a}$ relative to $u^{a}$ is given in terms of $x_{i}^{a}$ or $\mathfrak{e}_{i}^{a}, \mathfrak{b}_{i}^{a}$ by

$$
\begin{aligned}
v\left(e_{0}, u\right)^{a} & =-\frac{1}{2\left(u^{0}\right)^{2}} \sum_{(i j k)} \Im\left(x_{j}^{b}\left(\overline{x_{k}}\right)_{b}\right) x_{i}^{a}=\frac{1}{\left(u^{0}\right)^{2}} \sum_{(i j k)} \mathfrak{b}_{j}^{b}\left(\mathfrak{e}_{k}\right)_{b} \mathfrak{e}_{i}^{a} \\
& =\frac{1}{4 i\left(u^{0}\right)^{2}} \sum_{i=1}^{3}\left[x_{i}, \overline{x_{i}}\right]^{a}=\frac{1}{2\left(u^{0}\right)^{2}} \sum_{i=1}^{3}\left[\mathfrak{e}_{i}, \mathfrak{b}_{i}\right]^{a}
\end{aligned}
$$

\footnotetext{
${ }^{10}$ Given the first expression in 104 the first one in 105 is confirmed by $\left(e_{i}\right)_{a}=X_{a b}^{i} e_{0}^{b}=\mathcal{X}_{a b}^{i} e_{0}^{b}=2\left(u_{[a}\left(x_{i}\right)_{b]}\right)^{\dagger} e_{0}^{b}=$ $2 u_{[a}\left(x_{i}\right)_{b]} e_{0}^{b}+i\left[e_{0}, x_{i}\right]^{a}$, see 12 and $\left[78\right.$, and application of the identity 81 to $y^{a}=e_{0}^{a}$.
} 
where the square of the associated Lorentz factor is given by

$$
2\left(u^{0}\right)^{2}=\frac{1}{2} \sum_{i=1}^{3} x_{i}^{a}\left(\bar{x}_{i}\right)_{a}+\frac{1}{2}=\sum_{i=1}^{3} \mathfrak{e}_{i}^{a}\left(\mathfrak{e}_{i}\right)_{a}-1=\sum_{i=1}^{3} \mathfrak{b}_{i}^{a}\left(\mathfrak{b}_{i}\right)_{a}+2 .
$$

Hence the unique principal observer is $e_{0}^{a}=u^{0}\left[u^{a}+v\left(e_{0}, u\right)^{a}\right]$.

To obtain (111) one sums the first parts of (100) and (101) over $i$ and uses the second part of (97); the expressions (109) for $v\left(e_{0}, u\right)^{a}$ are immediate from the above; on applying the identity (81) to $y^{a}={\overline{x_{i}}}^{a}$ the first expressions in $(109)$ and 110 are seen to be equal, and then the second expression in 110 , follows from $\left[x_{i}, \bar{x}_{i}\right]^{a}=2 i\left[\mathfrak{e}_{i}, \mathfrak{b}_{i}\right]^{a}$.

By proposition 5.8 the problem of finding $v\left(e_{0}, u\right)^{a}$ is reduced to constructing the eigenvectors $x_{i}^{a}$ of Q. The projector of $\mathbb{C} u^{\perp}$ onto $\left\langle x_{i}^{a}\right\rangle$ is given by

$$
\left.\mathrm{R}^{i} \equiv \frac{\left(\mathrm{Q}-\lambda_{j} \mathrm{~h}\right)\left(\mathrm{Q}-\lambda_{k} \mathrm{~h}\right)}{\left(\lambda_{i}-\lambda_{j}\right)\left(\lambda_{i}-\lambda_{k}\right)}=\frac{\mathrm{Q}^{2}+\lambda_{i} \mathrm{Q}+\left(\lambda_{i}^{2}-I / 2\right) \mathrm{h}}{3 \lambda_{i}^{2}-I / 2} \Leftrightarrow \quad R^{i}\right)^{a}{ }_{b}=\frac{Q^{a}{ }_{c} Q^{c}{ }_{b}+\lambda_{i} Q^{a}{ }_{b}+\left(\lambda_{i}^{2}-I / 2\right) h_{b}^{a}}{3 \lambda_{i}^{2}-I / 2},
$$

as this operator annihilates $x_{j}^{a}, x_{k}^{a}$ and leaves $x_{i}^{a}$ invariant (see, e.g., appendix A of [52]). Take any $y^{a} \in u^{\perp}$ that is not an eigenvector of $\mathrm{Q}$ with eigenvalue $\lambda_{i}\left[y^{a} \notin\left\langle x_{i}^{a}\right\rangle \Leftrightarrow\left(R^{i}\right)^{a}{ }_{b} y^{b} \neq 0\right]$; for an arbitrary orthonormal tetrad $\left(u^{a}, e_{i^{\prime}}^{a}\right)$ at least one of the vectors $e_{i^{\prime}}^{a}$ may be taken as $y^{a}$. Then

$$
x_{i}^{a}=\frac{\left(R^{i}\right)_{b}^{a} y^{b}}{\sqrt{R_{c d}^{i} y^{c} y^{d}}} \quad\left[x_{i}^{a}=\frac{\left(R^{i}\right)_{i^{\prime}}^{m^{\prime}} e_{m^{\prime}}^{a}}{\sqrt{R_{i^{\prime} i^{\prime}}^{i}}}\right],
$$

where the result for $y^{a}=e_{i^{\prime}}^{a}$ is indicated between square brackets, and one applies (2) to $z=R_{c d}^{i} y^{c} y^{d}\left[R_{i^{\prime} i^{\prime}}^{i}\right]$. We conclude that an observer $u^{a}$ passing through a point $p$ and measuring $Q^{a b}=\left(E^{m^{\prime} n^{\prime}}-i H^{m^{\prime} n^{\prime}}\right) e_{m^{\prime}}^{a} e_{n^{\prime}}^{b}$ may check whether the gravitational field is of Petrov type I at $p$, and in this case determine the unique Weyl principal observer directly from $Q^{a b}$ by the following

Algorithm for obtaining the principal observer of a Petrov type I Weyl tensor, directly from electric and magnetic parts of the Weyl tensor measured by an arbitrary observer:

1. Compute $I$ and $J$ from $(65)$ and $(67)$; if $I^{3} \neq 6 J^{2}$ then the Petrov type is I at $p$, and go to step 2 .

2. Compute the eigenvalues $\lambda_{k}$ of $\mathrm{Q}$ from 166 , or from 180 when $q=0$.

3. For each $i \in\{1,2,3\}$ compute $\mathrm{R}^{i}$ from $(112)$, take $y^{a} \in u^{\perp}$ for which $\left(R^{i}\right)^{a}{ }_{b} y^{b} \neq 0$, and construct the unit eigenvectors $x_{i}^{a} \equiv \mathfrak{e}_{i}^{a}-i \mathfrak{b}_{i}^{a}$ of $\mathrm{Q}$ with eigenvalue $\lambda_{i}$ from 113.

4. The principal observer $e_{0}^{a}=u^{0}\left[u^{a}+v\left(e_{0}, u\right)^{a}\right]$ is given by 109) or (110), and 1111.

Likewise, given (111) the spatial principal tetrad vectors are found from 102 and 105 :

$$
e_{i}^{a}=\frac{1}{u^{0}}\left[\mathfrak{b}_{j}^{b}\left(\mathfrak{e}_{k}\right)_{b} u^{a}+\left(u^{0}\right)^{2} \mathfrak{e}_{i}^{a}+\mathfrak{b}_{i}^{b}\left(\mathfrak{e}_{j}\right)_{b} \mathfrak{b}_{j}^{a}+\mathfrak{b}_{i}^{b}\left(\mathfrak{e}_{k}\right)_{b} \mathfrak{b}_{k}^{a}\right] .
$$

Remark 5.9. In [53] Ferrando and Sáez gave a covariant algorithm to determine $e_{0}^{a}$ and $e_{i}^{a}$. In their approach one considers $X_{a b}^{i}=\Re\left(\mathcal{X}_{a b}^{i}\right)=2\left(e_{0}\right)_{[a}\left(e_{i}\right)_{b]}$ and obtains the projector $\left(P_{i}\right)^{a}{ }_{b}=\left(X^{i}\right)^{a}{ }_{c}\left(X^{i}\right)^{c}{ }_{b}=$ $-e_{0}^{a}\left(e_{0}\right)_{b}+e_{i}^{a}\left(e_{i}\right)_{b}$ on the blade $\Sigma_{i}$, see 78 and 16 with $e_{1}^{a}$ replaced by $e_{i}^{a}$; summing these projectors over $i$ yields $2 e_{0}^{a}\left(e_{0}\right)_{b}=\delta_{b}^{a}-\sum_{i=1}^{3}\left(P_{i}\right)_{b}^{a}$, and contraction with $u^{b}$ and normalization then gives $e_{0}^{a}$. On using the $i$-labeled version of (44) this gives back the final expression in (110), which is thus an explicit form of the expression for $e_{0}^{a}$ in Corollary 1 of [53]; once $e_{0}^{a}$ is found one gets the spatial princpal tetrad vectors simply from $e_{i}^{a}=\left(X^{i}\right)^{a}{ }_{b} e_{0}^{b}$ (see also footnote 10). This algorithm requires knowlegde of the eigenbivectors $\mathcal{X}_{a b}^{i}$ of the Weyl operator $\mathrm{C}$, which can be found by constructing analoga of the projectors (112) from C (see Proposition 1 of [53]) and applying these to a generic self-dual bivector; however, given 
$Q_{a b}=E_{a b}-i H_{a b}$ measured by an observer $u^{a}$ the operator C should be constructed first from (62) for this purpose. Our algorithm avoids this detour and the explicit use of bivectors, and finds the principal tetrad vectors directly from $Q_{a b}$, thereby staying entirely in the (complexified) rest space of $u^{a}$.

To deal with the other two points we derive expressions for the super-energy density and superPoynting vector. Following [13] we define the invariants

$$
r_{i} \equiv-\left[4 \Re\left(\lambda_{j} \bar{\lambda}_{k}\right)+\Re\left(\lambda_{k} \bar{\lambda}_{i}\right)+\Re\left(\lambda_{i} \bar{\lambda}_{j}\right)\right]=2\left(\left|\lambda_{j}\right|^{2}+\left|\lambda_{k}\right|^{2}\right)-\left|\lambda_{i}\right|^{2}=\left|\lambda_{j}-\lambda_{k}\right|^{2},
$$

which are related to the invariants $\xi$ and $q$ defined in 70 by

$$
\begin{aligned}
& 12 \xi=r_{1}+r_{2}+r_{3}, \\
& 36 q^{2}=2 r_{1} r_{2}+2 r_{2} r_{3}+2 r_{3} r_{1}-r_{1}^{2}-r_{2}^{2}-r_{3}^{2} .
\end{aligned}
$$

The non-defining equalities in 115) follow from $\lambda_{1}+\lambda_{2}+\lambda_{3}=0$; the last one was not observed in 13 . and makes clear that $r_{i}>0$ in the Petrov type I case. We will also consider the Petrov type D 'limit' (or subcase), to which all equations in this section apply as well if we choose, as before, a Weyl principal tetrad $\left(e_{0}^{a}, e_{i}^{a}\right)$ such that

$$
\text { [Petrov type D limit] } \quad \lambda_{2}=\lambda_{3}=-\lambda_{1} / 2 \equiv \lambda, \quad r_{1}=0, \quad r_{2}=r_{3}=6 \xi, \quad q=0 ;
$$

then the Weyl principal plane $\Sigma=\left\langle e_{0}^{a}, e_{1}^{a}\right\rangle$, and $u^{2}=u^{3}=0$ characterizes Weyl principal observers.

Substituting the decomposition 79 in 72 and 773 we obtain the super-energy density and superPoynting vector in terms of the orthonormal triad $\left(x_{i}^{a}\right)$ of $\mathbb{C} u^{\perp}$ :

$$
\begin{aligned}
\rho_{\mathrm{g}} & =\frac{1}{4} \sum_{m=1}^{3} \sum_{n=1}^{3} \lambda_{m} \overline{\lambda_{n}}\left[x_{m}^{a}\left(\overline{x_{n}}\right)_{a}\right]^{2}, \\
\mathcal{P}^{a} & =\frac{1}{4 i} \sum_{m=1}^{3} \sum_{n=1}^{3} \lambda_{m} \overline{\lambda_{n}}\left[\mathrm{x}_{m} \mathrm{x}_{m}, \overline{\mathrm{x}}_{n} \overline{\mathrm{x}}_{n}\right]^{a}=\frac{1}{4 i} \sum_{(i j k)} x_{i}^{a}\left(\lambda_{j}-\lambda_{k}\right) \sum_{n=1}^{3} \overline{\lambda_{n}} x_{j}^{b}\left(\overline{x_{n}}\right)_{b} x_{k}^{c}\left(\overline{x_{n}}\right)_{c},
\end{aligned}
$$

where we used (5) and (81) applied to $y^{a}={\overline{x_{n}}}^{a}$ for the last equality. Equations (99)-(100) then lead to the following expressions for $\rho_{\mathrm{g}}$ (cf. [13]) and the components of $\mathcal{P}^{a}=\mathcal{P}^{0} e_{0}^{a}+\mathcal{P}^{i} e_{i}^{a}$ in the principal tetrad 11

$$
\begin{aligned}
& \rho_{\mathrm{g}}=\xi+\Omega, \quad \Omega=A^{i} r_{i}+12 q u^{0} u^{1} u^{2} u^{3}, \quad A^{i}=\left(u^{0}\right)^{2}\left(u^{i}\right)^{2}-\left(u^{j}\right)^{2}\left(u^{k}\right)^{2}, \\
& 2 \mathcal{P}^{0}=u^{0}\left[r_{1}\left(u^{1}\right)^{2}+r_{2}\left(u^{2}\right)^{2}+r_{3}\left(u^{3}\right)^{2}-2 \Omega\right]+6 q u^{1} u^{2} u^{3}, \\
& 2 \mathcal{P}^{i}=u^{i}\left[r_{j}\left(u^{k}\right)^{2}+r_{k}\left(u^{j}\right)^{2}-r_{i}\left(u^{0}\right)^{2}-2 \Omega\right]-6 q u^{0} u^{j} u^{k},
\end{aligned}
$$

Applying (108) to $w^{a}=\mathcal{P}^{a}$ we obtain the expansion of $\mathcal{P}^{a}$ in the basis $\left(\mathfrak{e}_{i}^{a}\right)$ of $u^{\perp}$ :

$$
\mathcal{P}^{a}=\mathcal{P}^{i} \mathfrak{e}_{i}^{a} / u^{0} .
$$

Just as $(120)$ this expression makes explicit that the Poynting vector belongs to $u^{\perp}$ and connects with the (observer-dependent) eigenvectors $x_{i}^{a}$ of Q; however, it is manisfestly real and refers to the components relative to the canonical (observer-independent) orthonormal frame $\left(e_{0}^{a}, e_{i}^{a}\right)$, thus synthesizing both viewpoints.

\footnotetext{
${ }^{11}$ The expression 121 for $\rho_{\mathrm{g}}$ coincides with (C.5)-(C.6) of 13 except for the factor 12 in the second term of $\Omega$, which corrects the factor 6 in (C.5) and (C.7) of [13] and gives the correct equation (C.10) of [13]. Equations 122$)-(123)$ are new. An alternative way to derive [121)- 123 is by substituting [78 into 69, which yields [13, 43] $T^{a b c d}=\sum_{i=1}^{b}\left|\lambda_{i}\right|^{2} \Pi_{i}^{a b} \Pi_{i}^{c d}+$ $\sum_{(i j k)}\left(\lambda_{j} \overline{\lambda_{k}} \Pi_{j k}^{a b} \Pi_{j k}^{c d}+\overline{\lambda_{j}} \lambda_{k} \bar{\Pi}_{j k}^{a b} \bar{\Pi}_{j k}^{c d}\right)$ with $\Pi_{i}^{a b}=-e_{0}^{a} e_{0}^{b}+e_{i}^{a} e_{i}^{b}-\frac{1}{2} g^{a b}, \Pi_{j k}^{a b}=e_{j}{ }^{(a} e_{k}{ }^{b)}+i e_{0}{ }^{(a} e_{i}{ }^{b)}$, triply and quadruply contracting this with 975 and using $\rho_{\mathrm{g}}=T_{a b c d} u^{a} u^{b} u^{c} u^{d}$ and $\mathcal{P}^{a}=-T_{b c d}^{a} u^{b} u^{c} u^{d}-\rho_{\mathrm{g}} u^{a}$, see 74-73 and cf. remark 4.4
} 
In view of (97) the expression (121) give the super-energy density as a function of $\left(u^{1}, u^{2}, u^{3}\right) \in \mathbb{R}^{3}$. Note that this function is irrational when $q \neq 0$, and polynomial of degree 4 when $q=0$. The level surfaces of $\rho_{\mathrm{g}}$ for Petrov type I are rather complicated, in contrast to those in the Petrov type D limit (118), cf. remarks 4.5 and 5.6), for which one finds back (87) from (121) by identifying (52) with (97). However, by (75) and characterization (iv) of Weyl principal observers, and for all values of $q \in \mathbb{R}$ and of the triples $\left(r_{1}, r_{2}, r_{3}\right) \in \mathbb{R}_{>0}^{3}$ allowed by (117), $\rho_{\mathrm{g}}$ attains an absolute minimum value $\xi$ at $\left(u^{1}, u^{2}, u^{3}\right)=(0,0,0)$, corresponding to $u^{a}$ being the principal observer $e_{0}^{a}$ and to $\Omega=0$, while $\Omega>0$ when $\left(u^{1}, u^{2}, u^{3}\right) \neq(0,0,0)$. In appendix C1 1 we present a straightforward proof of this fact.

To what extent does a surrogate of theorem 5.5 hold? First note from 120 that in the type D limit (118) $\mathcal{P}^{a}$ is orthogonal to $x_{1}^{a} \equiv x^{a} \equiv \mathfrak{e}^{a}-i \mathfrak{b}^{a}$, such that $\mathcal{P}^{a} \propto[\mathfrak{e}, \mathfrak{b}]^{a} \propto v\left(u_{\Sigma}, u\right)^{a}$ and we retrieve the gravitational Wheeler analogue: for any $u^{a}$ there exists a principal observer (namely $u_{\Sigma}^{a}$ ) lying in $\left\langle u^{a}, \mathcal{P}^{a}\right\rangle$. In the Petrov type I case there is only one principal observer $e_{0}^{a}$ and the "maximal" surrogate of theorem 5.5 would be that, for any observer $u^{a}$, the velocity of $e_{0}^{a}$ relative to $u^{a}$ is proportional to the super-Poynting vector of $u^{a}, v\left(e_{0}, u\right)^{a} \propto \mathcal{P}^{a}$. However, this is not the case. Instead, it follows from 106 and (124) that

$$
v\left(e_{0}, u\right)^{a}=\alpha \mathcal{P}^{a} \quad \Leftrightarrow \quad u^{i}=-\alpha \mathcal{P}^{i}, \quad i=1,2,3 \quad \Leftrightarrow \quad \pi(u)^{a} \equiv u^{0} v\left(u, e_{0}\right)^{a}=-\alpha \pi(\mathcal{P})^{a} .
$$

Hence $v\left(e_{0}, u\right)^{a} \propto \mathcal{P}^{a}$ precisely when $v\left(u, e_{0}\right)^{a} \propto \pi(\mathcal{P})^{a}$, which is a condition in the rest space $e_{0}^{\perp}$ of the principal observer. From 125 it follows that

$$
u^{j} \mathcal{P}^{k}=u^{k} \mathcal{P}^{j}, \quad i=1,2,3,
$$

where $(i, j, k)$ is a cyclic permutation of $(1,2,3)$. Conversely, when $\mathcal{P}^{a} \neq 0$ equations (126) imply (125), with

$$
\alpha=\frac{-\mathcal{P}^{0}}{u^{0} \mathcal{P}^{a} \mathcal{P}_{a}}=\frac{-u^{i}}{\mathcal{P}^{i}}
$$

for any $i$ such that $\mathcal{P}^{i} \neq 0$, where the first equality follows from 125 and contracting 98 with $\mathcal{P}_{a}$. For Petrov type I the principal observer is unique and so $\mathcal{P}^{a}=0 \Leftrightarrow u^{a}=e_{0}^{a}$, such that 125$)$ is equivalent to (126). In the Petrov type D limit (118) we regard $e_{0}^{a}$ as a given principal observer, and on solving (126) we need to exclude the principal observers $u^{a} \neq e_{0}^{a}$, which are characterized by $u^{2}=u^{3}=0 \neq u^{1}$ but for which $v\left(e_{0}, u\right)^{a} \neq 0=\mathcal{P}^{a}$ and thus do not solve 125. For convenience we define

$$
a_{\beta} \equiv\left(u^{\beta}\right)^{2}, \beta=0,1,2,3 \quad \Rightarrow \quad a_{0}=1+a_{1}+a_{2}+a_{3},
$$

and infer from 123 that 126 translates to the conditions

$$
\begin{aligned}
u^{2} u^{3}\left[\left(a_{2}-a_{3}\right) r_{1}+\left(a_{0}+a_{1}\right)\left(r_{2}-r_{3}\right)\right] & =6 u^{0} u^{1}\left(a_{2}-a_{3}\right) q, \\
u^{3} u^{1}\left[\left(a_{1}-a_{3}\right) r_{2}+\left(a_{0}+a_{2}\right)\left(r_{1}-r_{3}\right)\right] & =6 u^{0} u^{2}\left(a_{1}-a_{3}\right) q, \\
u^{1} u^{2}\left[\left(a_{1}-a_{2}\right) r_{3}+\left(a_{0}+a_{3}\right)\left(r_{1}-r_{2}\right)\right] & =6 u^{0} u^{3}\left(a_{1}-a_{2}\right) q .
\end{aligned}
$$

In the Petrov type $D$ limit (118) these equations reduce to $u^{1} u^{2}=u^{1} u^{3}=0$, and by the above we find back the property (see remark 5.6) that for a given principal observer $e_{0}^{a}=E_{0}^{a}(\gamma)$ there is a $2 \mathrm{~d}$ variety $\mathcal{L}(\gamma)$ of observers $u^{a}$ satisfying $v\left(e_{0}^{a}, u\right)^{a} \propto \mathcal{P}^{a}$, namely those for which $u_{\Sigma}^{a}=e_{0}^{a}$, characterized by $u^{1}=0$. For Petrov type I the situation is different. Clearly, at most two of the equations (129)-131) can be independent (when $u^{3} \neq 0$ the last equation is a consequence of the first and the second, and analogously when $u^{1} \neq 0$ or $u^{2} \neq 0$ ). A pair of non-trivial equations corresponds to the intersection $\mathcal{I}$ of two $2 \mathrm{~d}$ surfaces in $U^{+}$. Take the square of (129) and (130), eliminate $q^{2}$ by (117) and $a_{0}$ by (128). This yields two polynomial equations $K\left(a_{i} \mid r_{j}\right)=0$ and $L\left(a_{i} \mid r_{j}\right)=0$ in the variables $a_{i}>0$, where the $r_{j}$ are regarded as parameters $(i, j=1,2,3)$. Computing the resultant of $K\left(a_{i} \mid r_{j}\right)$ and $L\left(a_{i} \mid r_{j}\right)$ with respect to $a_{3}$ leads to a non-trivial polynomial equation $M\left(a_{1}, a_{2} \mid r_{j}\right)=0$, for any values of the parameters $r_{j}>0$. For each solution $\left(a_{1}, a_{2}\right) \in \mathbb{R}_{\geq 0}^{2}$ of this equation there is a finite number of values $a_{3} \in \mathbb{R}_{\geq 0}$ for which $K\left(a_{i} \mid r_{j}\right)=0$ and $L\left(a_{i} \mid r_{j}\right)=0$, leading to a finite union of curves and isolated points in $\mathbb{R}_{\geq 0}^{3} \ni\left(a_{1}, a_{2}, a_{3}\right)$, and the intersection $\mathcal{I}$ forms a part hereof. Note that when two of the $u^{i}$ s are zero the system (129)-(131) is identically satisfied, irrespective of $q$ and $r_{i}$. Hence we have 
Theorem 5.10. Suppose the Weyl tensor is of Petrov type I at a point $p$, and let $\left(e_{0}^{a}, e_{i}^{a}\right)$ be the principal tetrad at $p$. Then the observers $u^{a}=u^{0} e_{0}^{a}+u^{i} e_{i}^{a}$ for which (129)-(131) and thus $v\left(e_{0}, u\right)^{a} \propto \mathcal{P}^{a}$ holds form a $1 d$ variety (finite union of curves and points) in $U^{+}$. This variety always contains (among others) three canonical curves of observers, given by

$$
u^{a}=\cosh (\varphi) e_{0}^{a}+\sinh (\varphi) e_{i}^{a}, \quad \varphi \in \mathbb{R}, \quad i \in\{1,2,3\}
$$

and obtained by boosting the principal observer $e_{0}^{a}$ arbitrarily along one of the spatial tetrad vectors $e_{i}^{a}$.

Remark 5.11. Consider the special observers 132. For fixed $i \in\{1,2,3\}$ we have $u^{0}=\cosh (\varphi), u^{i}=$ $\sinh (\varphi), u^{j}=u^{k}=0$, and find

$$
\rho_{\mathrm{g}}=\xi+\frac{1}{4} r_{i} \sinh (2 \varphi)^{2}, \quad \mathcal{P}^{a}=-\frac{1}{8} r_{i} \sinh (4 \varphi) \mathfrak{e}_{i}^{a}, \quad v\left(e_{0}, u\right)^{a}=-\tanh (\varphi) \mathfrak{e}_{i}^{a}
$$

from 106, 121 and 123-124, such that 125 is realized by

$$
v\left(e_{0}, u\right)^{a}=\frac{4 \mathcal{P}^{a}}{r_{i} \zeta_{i}\left(\zeta_{i}+1\right)}, \quad \zeta_{i}=\sqrt{1+\frac{4\left(\rho_{\mathrm{g}}-\xi\right)}{r_{i}}}=\cosh (2 \varphi)
$$

In the Petrov type D limit (118) the expressions 133 also apply to observers obtained by boosting any principal observer $e_{0}^{a} \in \Sigma$ along the spatial vectors of a principal tetrad $\left(e_{0}^{a}, e_{i}^{a}\right)$. In [26] Bini et al. considered such observers for both Petrov types I and D, and 133 explicates their two main results: $v\left(e_{0}, u\right)^{a} \propto \mathcal{P}^{a}$ holds, and for varying $\varphi$ the super-energy density attains its minimum at $\varphi=0$, which is a special case of characterization (iv) of a principal observer $e_{0}^{a}$; note that the first equation of 133 can be written as $\rho_{\mathrm{g}}=\frac{1}{24}\left(2 r_{j}+2 r_{k}-r_{i}\right)+\frac{1}{8} r_{i} \cosh (4 \varphi)$, which is a more explicit form of Eq. (42) of [26]. In the Petrov type D limit (118) we can identify (97) with (52); for $i=2\left(u^{1}=u^{3}=0\right)$ and $i=3\left(u^{1}=u^{2}=0\right)$ we have $\varphi= \pm \psi_{\Sigma}, \gamma=0$ and $\vartheta=0$, resp. $\vartheta=\frac{\pi}{2}$, and retrieve from (133)-(134) that (87)-(88) is valid, such that $\rho_{\mathrm{g}}$ increases with $|\varphi|=\psi_{\Sigma}$; for $i=1\left(u^{2}=u^{3}=0\right)$, however, we have $\psi_{\Sigma}=0$ : for all values of $\varphi=\gamma$ the observers (132) with $i=1$ belong to $\Sigma$, are thus principal $\left(\mathcal{P}^{a}=0\right)$, and have the same minimal super-energy density $\rho_{\mathrm{g}}^{1}(\varphi)=\xi$, in agreement with characterizations (ii)' and (iv) of principal observers. This amends the statement at the end of Sec. 5 of [26] when applied to Petrov type $\mathrm{D}{ }^{12}$

In the Petrov type I case the special observers 132 may be characterized as follows. For an arbitrary observer $u^{a}$ given by (97) the orthogonal eigendirections of the endomorphism Q associated to an observer $u^{a}$ are generated by the three vectors $x_{i}^{a}$ given in (99). If $u^{j}=u^{k}=0$ (for some $i \in\{1,2,3\}$, with $(i, j, k$ ) a cyclic permuation of $(1,2,3))$ then $\mathfrak{b}_{i}^{a}=0$ and so $x_{i}^{a}=\mathfrak{e}_{i}^{a}$ spans a real direction in $\mathbb{C} u^{\perp}$. Conversely, if $x_{i}^{a}$ spans a real direction then $\mathfrak{e}_{i}^{a}$ and $\mathfrak{b}_{i}^{a}$ must be linearly dependent; by $\mathfrak{e}_{i}^{a} \neq 0$ and $\mathfrak{e}_{i}^{a}\left(\mathfrak{b}_{i}\right)_{a}=0$ (see (101)) this implies $\mathfrak{b}_{i}^{a}=0$, whence $x_{i}^{a}=\mathfrak{e}_{i}^{a}$ and $u^{j}=u^{k}=0$ from (99). Combined with (133) and by the same argument as in remark 5.7 we conclude that for a non-principal observer $u^{a}$ the endomorphism $\mathrm{Q}=\mathrm{E}-i \mathrm{H}$ has no real eigendirections except when $u^{a}$ is one of the observers 132 for some $i \in\{1,2,3\}$, in which case $\mathcal{P}^{a}$ is an eigenvector of $\mathrm{Q}$ with eigenvalue $\lambda_{i}$ (and thus of $\mathrm{E}$ and $\mathrm{H}$ with respective eigenvalues $\Re\left(\lambda_{i}\right)$ and $-\Im\left(\lambda_{i}\right)$ ) and $\left\langle\mathcal{P}^{a}\right\rangle$ is the only real eigendirection of $\mathrm{Q}$ and the only common eigendirection of $\mathrm{E}$ and $\mathrm{H}$. This relates the observers 132 to general observers in the Petrov type D case, cf. remark 5.7. In fact, in the Petrov type D limit (118) one can write any given observer as $u^{a}=\cosh \left(\psi_{\Sigma}\right) u_{\Sigma}^{a}+\sinh \left(\psi_{\Sigma}\right) u_{\perp}^{a}$ and identify $\left(e_{0}^{a}, e_{1}^{a}, e_{2}^{a}, e_{3}^{a}\right)$ with the principal tetrad $\left(u_{\Sigma}^{a}, \mathfrak{e}_{\Sigma}^{a}, u_{\perp}^{a}, e_{\perp}^{a}\right)$, see (36) and 40p; hence $u^{1}=u^{\overline{3}}=0$ and $u^{a}$ takes the form (132) with $i=2$ and $\varphi= \pm \psi_{\Sigma}$, such that $\lambda_{i}=\lambda$ and one recovers remark 5.7 .

The full $1 \mathrm{~d}$ variety of theorem 5.10 contains other observers than 132 , its shape depending on the values of $q$ and $r_{i}$. Below we work out the two cases corresponding to degenerate Petrov type I in the extended Petrov classification by Arianrhod and McIntosh [27] (see appendix B.2). To find the extra observers $u^{a}$ we require throughout that at most one of the components $u^{i}$ vanishes. Figures 3 and 4 provide illustrations.

\footnotetext{
${ }^{12}$ We note that Eqs. (39)-(42) of [26] do not apply to boosts along $e_{1}^{a}$ in the Petrov type D limit [118], since $\widehat{v}\left(u^{\prime}, u\right)^{a} \equiv e_{1}^{a}$ and thus, in the notation of [26], $Z^{\perp \perp(\mathrm{TF})}(u)=0$.
} 

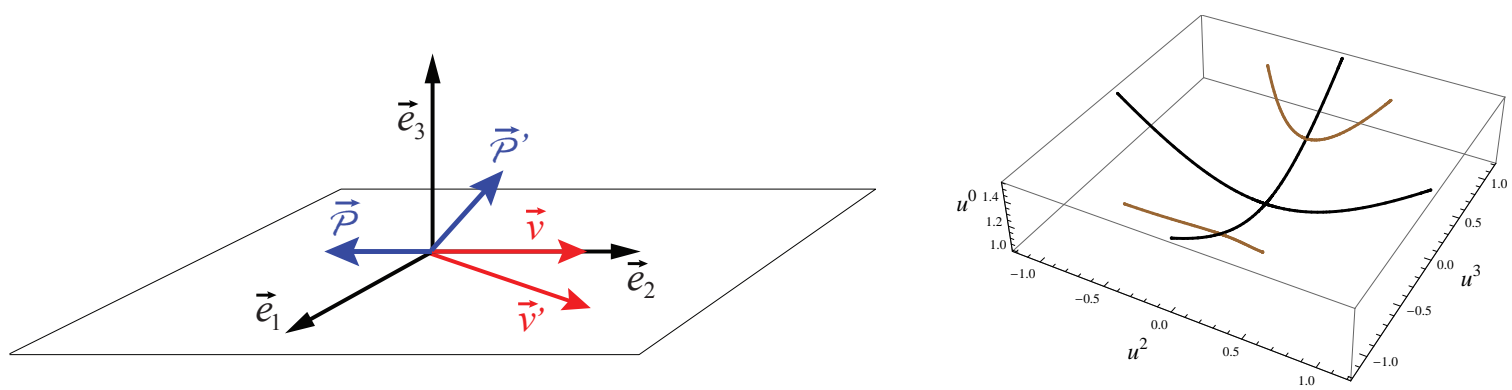

Figure 3: Left panel: the situation in the rest space $e_{0}^{\perp}$ of the unique principal observer $e_{0}^{a}$ in a Petrov type I spacetime. (Arrows denote projected vectors onto $e_{0}^{\perp}$.) The vectors $\vec{e}_{i}$ span the spatial principal directions. Observers moving (with relative velocity $\vec{v} \equiv \vec{v}\left(u, e_{0}\right)$ ) along one of the $\vec{e}_{i}$ measure a superPoynting vector whose projection onto $e_{0}^{\perp}, \overrightarrow{\mathcal{P}} \equiv \pi(\mathcal{P})^{a}$, is along $\vec{e}_{i}$; hence their boost back to the principal observer is along $\mathcal{P}^{a}: v\left(e_{0}, u\right)^{a} \propto \mathcal{P}^{a}$. For a generic observer $u^{\prime a}$ this does not hold: $v\left(e_{0}, u^{\prime}\right)^{a} \not \mathcal{P}^{\prime a}$. Right panel: the spacetime curves of observers $u^{a}$ for which $v\left(e_{0}, u\right)^{a} \propto \mathcal{P}^{a}$, represented in the 3d diagram $e_{1}^{\perp} \Leftrightarrow u^{1}=0$, in the case where $q=0$ and $0 \neq\left|\lambda_{1}\right|<\left|\lambda_{2}\right|<\left|\lambda_{3}\right|$. The black curves are the hyperbolae of observers moving along $e_{2}^{a}$ and $e_{3}^{a}$; note that the observers moving along $e_{1}^{a}$ (with $u^{2}=u^{3}=0$ ) cannot be represented in this diagram. The two brown curves represent the 'extra' observers for which $v\left(e_{0}, u\right)^{a} \propto \mathcal{P}^{a}$ in the $q=0$ case, corresponding to Eq. 136).

Example 5.12. The case $q=0$. This covers precisely types $\mathrm{I}\left(M^{+}\right)$and $\mathrm{I}\left(M^{\infty}\right)$ in the extended Petrov classification, see appendix $\overline{B .2}$ and remark 5.3 By proposition B.1 there is a unique eigenvalue with smallest modulus, say $\lambda_{1}$, and the Weyl PNDs span the $3 \mathrm{~d}$ space $e_{1}^{-}=\left\langle e_{0}^{a}, e_{2}^{a}, e_{3}^{a}\right\rangle$. We order the principal tetrad vectors $e_{2}^{a}$ and $e_{3}^{a}$ such that $\left|\lambda_{1}\right|<\left|\lambda_{2}\right| \leq\left|\lambda_{3}\right|$; then the types $\mathrm{I}\left(M^{+}\right)$and $\mathrm{I}\left(M^{\infty}\right)$ correspond to $\lambda_{1} \neq 0 \Leftrightarrow\left|\lambda_{2}\right|<\left|\lambda_{3}\right|$ and $\lambda_{1}=0 \Leftrightarrow\left|\lambda_{2}\right|=\left|\lambda_{3}\right| \Leftrightarrow r_{2}=r_{3}$, respectively. By (181) we have

$$
\sqrt{r_{1}}=\sqrt{r_{2}}+\sqrt{r_{3}} \neq 0 \quad\left(\left|\lambda_{3}-\lambda_{2}\right|=\left|\lambda_{3}-\lambda_{1}\right|+\left|\lambda_{2}-\lambda_{1}\right| \neq 0\right),
$$

whence $r_{1}>r_{2}+r_{3}$, and so by (128) the factors between square brackets in the left hands of Eqs. (130) and (131) exceed $\left(1+2 a_{1}+2 a_{2}\right) r_{2}>0$ and $\left(1+2 a_{1}+2 a_{3}\right) r_{3}>0$, respectively. Hence these equations are equivalent to $u^{1} u^{2}=u^{1} u^{3}=0$; by our requirement that at most one $u^{i}$ vanishes, and using (128) and (135), we find that Eqs. (129)-(131) reduce to

$$
\begin{aligned}
& u^{1}=0, \quad 2 \sqrt{r_{3}} a_{3}-2 \sqrt{r_{2}} a_{2}=\sqrt{r_{2}}-\sqrt{r_{3}}, \\
& \text { i.e. } \quad u^{1}=0, \quad 2\left(\left|\lambda_{2}\right|-\left|\lambda_{1}\right|\right)\left(u^{3}\right)^{2}-2\left(\left|\lambda_{3}\right|+\left|\lambda_{1}\right|\right)\left(u^{2}\right)^{2}=3\left|\lambda_{1}\right| \text {, }
\end{aligned}
$$

where we have also used (181). Thus the extra observers form two curves lying in $e_{1}^{\perp}$. In the type $\mathrm{I}\left(M^{+}\right)$case the pair $\left(u^{2}, u^{3}\right) \in \mathbb{R}^{2}$ lies on one of the two branches of a hyperbola, parametrized by $\left(u^{2}, u^{3}\right)=(b \sinh (\varphi), \pm a \cosh (\varphi)), \varphi \in \mathbb{R}$, where $a^{-2}=\frac{2}{3}\left(\left|\lambda_{2}\right| /\left|\lambda_{1}\right|-1\right), b^{-2}=\frac{2}{3}\left(\left|\lambda_{3}\right| /\left|\lambda_{1}\right|+1\right)$; note that the major axis corresponds to the eigenvalue $\lambda_{3}$ with largest modulus, and $\left|u^{2}\right|<\left|u^{3}\right|$ because of $a>b \Leftrightarrow r_{3}<r_{2}$ (see (181)); this case is illustrated in the right panel of Fig. 3 . In the type $\mathrm{I}\left(M^{\infty}\right)$ case the hyperbola degenerates to $u^{3}= \pm u^{2}$, such that the curves consist of the observers $u^{a}=\cosh (\varphi) e_{0}^{a}+\sinh (\varphi)\left[e_{2}^{a} \pm e_{3}^{a}\right] / \sqrt{2}, \varphi \in \mathbb{R}$ obtained by boosting $e_{0}^{a}$ arbitrarily along the two bisectors $\left\langle e_{2}^{a} \pm e_{3}^{a}\right\rangle$ of $\left\langle e_{2}^{a}\right\rangle$ and $\left\langle e_{3}^{a}\right\rangle$. From (121)-(128) and 1135)-126) one finds, for both types $\mathrm{I}\left(M^{+}\right)$and $\mathrm{I}\left(M^{\infty}\right)$ :

$$
\rho_{\mathrm{g}}-\xi=r_{2} a_{2}+r_{3} a_{3}+\frac{\left(\sqrt{r_{2}}-\sqrt{r_{3}}\right)^{2}}{4}=\sqrt{r_{2} r_{3}} a_{0}-\frac{r_{1}}{4}, \quad v\left(e_{0}, u\right)^{a}=\frac{2 \mathcal{P}^{a}}{\sqrt{r_{2} r_{3}} a_{0}}=\frac{8 \mathcal{P}^{a}}{4\left(\rho_{\mathrm{g}}-\xi\right)+r_{1}} .
$$

Conversely, compatibility of (129)-(131) with $u^{1}=0 \neq u^{2} u^{3}$ or a cyclic permutation hereof requires $q=0$. Hence, when $q \neq 0$ the extra observers have $u^{1} u^{2} u^{3} \neq 0$. 


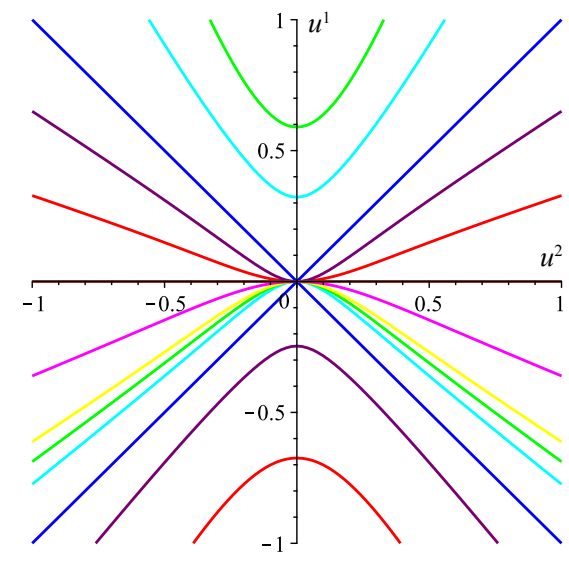

Figure 4: Projections of the curves of extra observers $u^{a}$ satisfying $v\left(e_{0}, u\right)^{a} \propto \mathcal{P}^{a}$ onto the $\left(u^{2}, u^{1}\right)$-plane in the degenerate Petrov type I case $r_{2}=r_{3}, r_{1}=k r_{2}, q \geq 0$, corresponding to Eq. (138) with $\delta=1$, for the values $k=0.2$ (red), $k=0.6$ (purple), $k=1$ (blue), $k=1.5$ (cyan), $k=1.75$ (green), $k=2$ (yellow), $k=3$ (magenta) and $k=4 \Leftrightarrow q=0$ (brown). Mirroring about the $u^{2}$-axis yields the projections of the curves corresponding to $\delta=-1$, for the same values of $k$ and $q$. For $q \leq 0$ the displays correspond to $\delta=-1$ instead, and the $u^{2}$-mirrors of these to $\delta=1$. In general, for $0<k<2$ and $2 \leq k \leq 4$ this yields, respectively, four and two spacetime curves $u^{a}=\sqrt{1+\left(u^{1}\right)^{2}+2\left(u^{2}\right)^{2}} e_{0}^{a}+u^{1} e_{1}^{a}+u^{2}\left(e_{2}^{a}+\delta e_{3}^{a}\right)$ of extra observers satisfying $v\left(e_{0}, u\right)^{a} \propto \mathcal{P}^{a}$, different from the special observers 132 but including $e_{0}^{a}$ (corresponding to the origin $u^{2}=u^{1}=0$ ).

Example 5.13. The case $r_{2}=r_{3} \Leftrightarrow\left|\lambda_{2}\right|=\left|\lambda_{3}\right|$. This is type $\mathrm{I}\left(M^{-}\right)$in the extended Petrov classification. By (117) one has $36 q^{2}=r_{1}\left(4 r_{2}-r_{1}\right)$ and so $q=\operatorname{sgn}(q) \sqrt{k(4-k)} r_{2} / 6$ with $0<k \equiv r_{1} / r_{2} \leq 4$. The subcase $q=0 \Leftrightarrow k=4$ corresponds to $\lambda_{1}=0$ and gives $a_{1}=0$ and $a_{2}=a_{3}$, cf. supra. Take now $q \neq 0$ and suppose that (129)-(131) holds with $a_{2} \neq a_{3}$ (implying $u^{1} u^{2} u^{3} \neq 0$ ). Eq. (129) then gives $u^{2} u^{3} r_{1}=6 u^{0} u^{1} q$; taking the square hereof yields $\left(a_{0} a_{1}+a_{2} a_{3}\right) k=4 a_{0} a_{1}$, while compatibility with 130 requires $\left(a_{0} a_{1}+a_{2} a_{3}\right) k=a_{1}\left(a_{0}+a_{2}+a_{3}-a_{1}\right)$; hence $2 a_{0}+2 a_{1}+1=0$ by (128), a contradiction. Hence $a_{2}=a_{3}$ and it follows that, when at most one $u^{i}$ vanishes, the system 129-1131) is equivalent to

$$
u^{3}=\delta u^{2}, \delta= \pm 1, \quad \operatorname{sgn}(q) \sqrt{k(4-k)\left(1+a_{1}+2 a_{2}\right)}\left(a_{1}-a_{2}\right)=\delta u^{1}\left[k a_{1}+(3 k-4) a_{2}+k-1\right] .
$$

A detailed analysi: ${ }^{13}$ shows that for $k \neq 1$ the second part of 138 is solved by $u^{1}=\epsilon f\left(u^{2}\right)$ and additionally by $u^{1}=-\epsilon g\left(u^{2}\right)$ when $0<k<2$, where $\epsilon=\operatorname{sgn}(q(1-k)) \delta$ and $f, g$ are smooth, even functions satisfying $0<f\left(u^{2}\right)<\left|u^{2}\right|<g\left(u^{2}\right), u^{2} \neq 0$ and $0=f(0)<g(0)$. For $k=1 \Leftrightarrow r_{1}=r_{2}=r_{3} \Leftrightarrow$ $I=0$ one has $u^{1}= \pm u^{2}= \pm u^{3}$, as expected by symmetry and corresponding to the non-smooth limit $f\left(u^{2}\right)=g\left(u^{2}\right)=\left|u^{2}\right|$ in the above; in this case one has

$$
v\left(e_{0}, u\right)^{a}=\alpha \mathcal{P}^{a}, \quad 1 / \alpha=2 \xi u^{0}\left(a_{0}+a_{1}\right)\left[u^{0}+\operatorname{sgn}\left(q u^{1}\right) \delta \sqrt{3 a_{1}}\right], \quad u^{0}=\sqrt{1+3 a_{1}} .
$$

In the limit $k=4 \Leftrightarrow q=0$ one finds back $u^{1}=0, u^{3}= \pm u^{2}$ (corresponding to $f=0$ ). Hence there are four and two smooth curves of extra observers when $0<k<2$ and $2 \leq k \leq 4$, respectively; see Fig. 4 .

Conversely, compatibility of $(129)$ with $a_{2}=a_{3}$ requires $r_{2}=r_{3}$. Hence, when $r_{2} \neq r_{3}$ the extra observers have $u^{2} \neq \pm u^{3}$, and analogously $r_{1} \neq r_{2} \Rightarrow u^{1} \neq \pm u^{2}$ and $r_{1} \neq r_{3} \Rightarrow u^{1} \neq \pm u^{3}$.

\footnotetext{
${ }^{13}$ Taking the square of the second equation gives a polynomial equation $P\left(a_{1} \mid a_{2}, k\right)=0$ of degree 3 in $a_{1}$, where $a_{2}$ and $k$ are viewed as parameters, and discriminant theory can be used (see e.g. theorem 1 in [54]).
} 
Remark 5.14. Consider a principal observer $e_{0}^{a}$ and an arbitrary non-principal observer $u^{a}$. The condition $v\left(e_{0}, u\right)^{a} \propto \mathcal{P}^{a}$ can also be written as

$$
v\left(e_{0}, u\right)^{a} \propto \mathcal{P}^{a} \quad \Leftrightarrow \quad e_{0}^{a} \in\left\langle u^{a}, \mathcal{P}^{a}\right\rangle \quad \Leftrightarrow \quad D^{a} \equiv\left[e_{0}, \mathcal{P}\right]^{a} \equiv \epsilon_{b c d}^{a} u^{d} \mathcal{P}^{c} e_{0}^{b} \equiv \frac{1}{2}[\mathrm{E}, \mathrm{H}]_{b}^{a} e_{0}^{b}=0,
$$

and thus boils down to the vectors $e_{0}^{a}, u^{a}, \mathcal{P}^{a}$ being linearly dependent (i.e., belonging to a 2-plane), or to $e_{0}^{a}$ belonging to the kernel of $[\mathrm{E}, \mathrm{H}]^{a}{ }_{b}$. However, according to theorem 5.10 and the text preceeding it, (140) does not hold for almost all observers $u^{a}$ in the type I case, and evenly so in the type $\mathrm{D}$ case if we consider $e_{0}^{a}$ as a given principal observer, leading to a non-vanishing vector $D^{a}=u^{0} \epsilon^{a}{ }_{b c d} u^{d} v\left(e_{0}, u\right)^{b} \mathcal{P}^{c}$. In general, one can decompose $v\left(e_{0}, u\right)^{a}$ along and orthogonal to $\mathcal{P}^{a}$; one easily finds

$$
v\left(e_{0}, u\right)^{a}=v_{\| \mathcal{P}}^{a}+v_{\perp \mathcal{P}}^{a}=-\frac{\mathcal{P}^{0} \mathcal{P}^{a}}{u^{0} \mathcal{P}^{b} \mathcal{P}_{b}}+\frac{\epsilon_{b c d}^{a} \mathcal{P}^{b} D^{c} u^{d}}{u^{0} \mathcal{P}^{b} \mathcal{P}_{b}}
$$

[compare to (125) and (127]]. In appendix C2 2 it is proven that

$$
\mathcal{P}^{a} \neq 0 \quad \Rightarrow \quad-u^{0} \mathcal{P}^{0}>0,
$$

saying that for a given non-principal observer $u^{a}$ and principal observer $e_{0}^{a}$ the relative velocity $v\left(e_{0}, u\right)^{a}$ always has a non-zero, positive component along $\mathcal{P}^{a}$; i.e., $v_{\| \mathcal{P}}^{a}=\alpha \mathcal{P}^{a}$ with $\alpha>0$. Note that the formula 92 in the Petrov type D case is a special instance of 141 , with $v_{\perp \mathcal{P}}^{a}=v_{\| \mathfrak{e}}^{a}$ and $e_{0}^{a}=u^{\prime a}=u^{\prime a}(\varphi)$, while (88), 137) and 139) confirm 142.

\section{Examples}

\subsection{Electromagnetic field of a spinning charge}

The EM field generated by a charged spinning body with charge $Q \neq 0$ and dipole moment of magnitude $\mu$ oriented along the $z$-axis, in flat spacetime, is described in spherical coordinates $\left[d s^{2}=\right.$ $\left.-d t^{2}+d r^{2}+r^{2}\left(\sin (\theta)^{2} d \phi^{2}+d \theta^{2}\right)\right]$ by the vector potential $A^{a}=(Q / r) \partial_{t}^{a}+\left(\mu / r^{3}\right) \partial_{\phi}^{a}$. Consider the following orthonormal vector basis:

$$
e_{0}^{a}=\partial_{t}^{a}, \quad e_{r}^{a}=\partial_{r}^{a}, \quad e_{\theta}^{a}=\frac{1}{r} \partial_{\theta}^{a}, \quad e_{\phi}^{a}=\frac{1}{r \sin (\theta)} \partial_{\phi}^{a} .
$$

The electric and magnetic fields measured by the laboratory (or 'static') observers $u_{\text {lab }}^{a}=\partial_{t}^{a}$ are

$$
E^{a}=\frac{Q}{r^{2}} e_{r}^{a}, \quad B^{a}=\frac{\mu}{r^{3}}\left[2 \cos (\theta) e_{r}^{a}+\sin (\theta) e_{\theta}^{a}\right] .
$$

Assuming $r>a \equiv 2 \mu / Q$ (see footnote 10 of [37]) the field is everywhere non-null. The Poynting vector measured by $u_{\text {lab }}^{a}$ is

$$
p^{a}=\frac{Q \mu \sin (\theta)}{4 \pi r^{5}} e_{\phi}^{a} .
$$

Hence there is a non-vanishing flux of EM field energy; such energy flows stationarily around closed loops parallel to the equatorial plane, as illustrated in Fig. 5 a.

According to (49) and (57) the EM principal observers $u^{\prime a}$ (for which $p^{\prime a}=0$ ) move relative to $u_{\text {lab }}^{a}$ with a velocity that has a fixed component parallel to $p^{a}$ given by $v_{\| p}^{a} \equiv p^{a} /\left(\rho_{\mathrm{em}}+\chi\right)$, where

$$
\rho_{\mathrm{em}}=\frac{Q^{2} r^{2}+\mu^{2}\left(1+3 \cos ^{2}(\theta)\right)}{8 \pi r^{6}} ; \quad \chi=\frac{\left|I_{F}\right|}{8 \pi}, \quad I_{F}=\frac{Q^{2} r^{2}-\mu^{2}\left(1+3 \cos ^{2}(\theta)\right)-4 i Q r \mu \cos (\theta)}{r^{6}},
$$

which amounts to an angular velocity $v_{\| p}^{\phi}=u^{\prime \phi} / u^{\prime t} \equiv \omega_{\left(p^{\prime}=0\right)}$ given by

$$
\omega_{\left(p^{\prime}=0\right)}=\frac{2 Q \mu}{Q^{2} r^{2}+\mu^{2}\left(1+3 \cos ^{2}(\theta)\right)+\sqrt{\left(Q^{2} r^{2}-\mu^{2}\right)^{2}+2 \mu^{2}\left(5 q^{2} r^{2}+3 \mu^{2}\right) \cos ^{2}(\theta)+9 \mu^{2} \cos ^{4}(\theta)}} .
$$



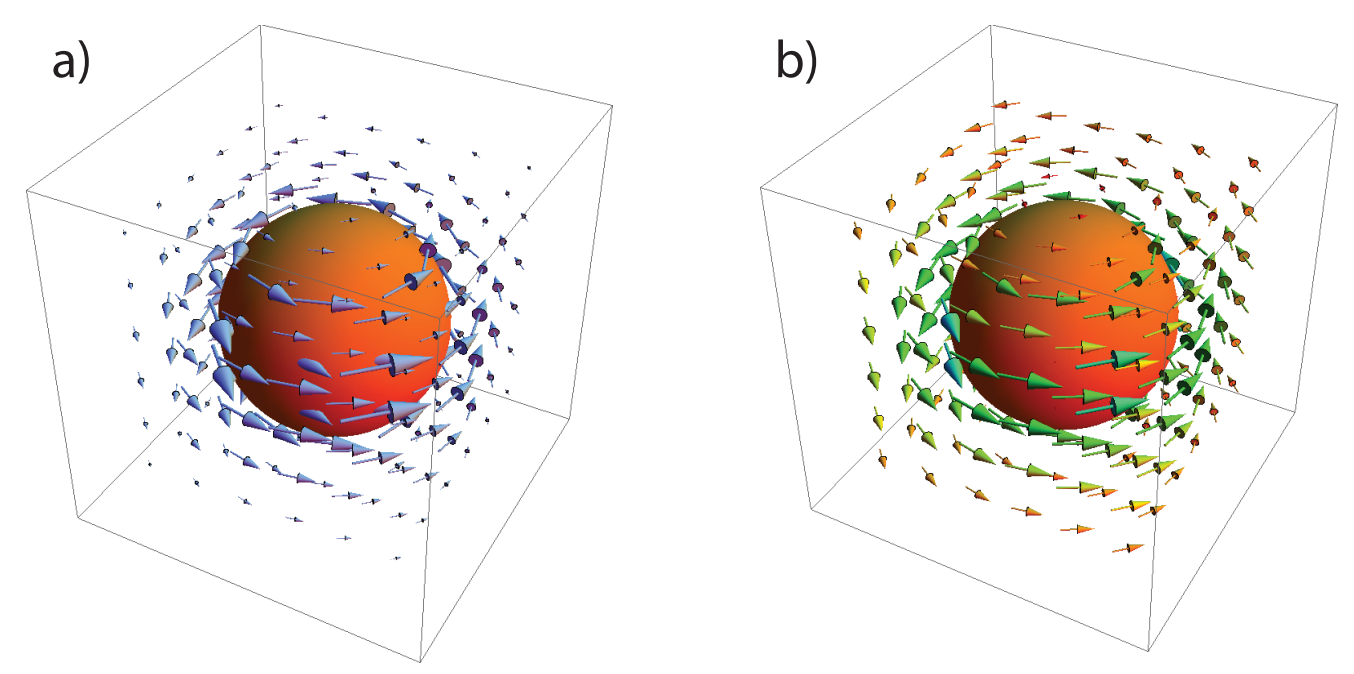

Figure 5: a) Poynting vector $p^{a}=p^{\phi} \partial_{\phi}^{a}$ for the electromagnetic field of a spinning charge, as measured by the static observers $u_{\text {lab }}^{a}=\partial_{t}^{a}$ (the "laboratory" frame); the field energy flows stationarily along circular loops parallel to the equatorial plane. b) The principal observers $u^{\prime a}$, for which the Poynting vector vanishes, have a velocity with a component $v_{\| p}^{a}=\omega_{\left(p^{\prime}=0\right)} \partial_{\phi}^{a}$ along $p^{a}$ given by 146 , and an arbitrary component along $\mathfrak{e}^{a}$. The special case of the canonical principal observers $u^{\prime a}=u_{\Sigma}^{a}$, moving along circles tangent to $p^{a}$ (i.e., $v^{a}=v_{\| p}^{a}$ ), is represented.

The relative velocity $v\left(u^{\prime}, u\right)^{a}$ may also have an arbitrary component $v_{\| \mathfrak{e}}^{a}$ parallel to the vector $\mathfrak{e}^{a}$, as given in (58). In the special case of the canonical principal observers $u^{\prime a}=u_{\Sigma}^{a}$ for which $v_{\| \mathfrak{e}}^{a}=0 \Leftrightarrow$ $v^{a}=v_{\| p}^{a}$ (which are observers in circular motion), this velocity field is illustrated in Fig. 5 p. Finally, in the equatorial plane $\theta=\pi / 2$, which is a purely electric region, we have $\omega_{\left(p^{\prime}=0\right)}=\mu /\left(Q r^{2}\right)$, and $\mathfrak{e}^{a}=E^{a} r^{3} / \sqrt{Q^{2} r^{2}-\mu^{2}}$, yielding the velocities of the observers measuring $B^{\prime a}=0$ obtained in [37].

\subsection{Kerr-Newman spacetimes}

Consider a charged rotating Kerr-Newman black hole in Boyer-Lindquist coordinates outside the event horizon, $r>M+\sqrt{M^{2}-\left(a^{2}+Q^{2}\right)}$, where the mass $M$, angular momentum per unit mass $a$ and charge $Q$ satisfy $M^{2}>a^{2}+Q^{2}$, and with $0<\theta<\pi$. The metric is $g_{a b}=-\Omega_{a}^{0} \Omega_{b}^{0}+\Omega_{a}^{1} \Omega_{b}^{1}+\Omega_{a}^{2} \Omega_{b}^{2}+\Omega_{a}^{3} \Omega_{b}^{3}$ with

$$
\begin{aligned}
& \Omega_{a}^{0}=\frac{\sqrt{\Delta}}{\rho}\left(d_{a} t-a \sin ^{2}(\theta) d_{a} \phi\right), \quad \Omega_{a}^{1}=\frac{\rho}{\sqrt{\Delta}} d_{a} r, \quad \Omega_{a}^{2}=\frac{\sin (\theta)}{\rho}\left[a d_{a} t-\left(r^{2}+a^{2}\right) d_{a} \phi\right], \quad \Omega_{a}^{3}=\rho d_{a} \theta \\
& \rho^{2} \equiv r^{2}+a^{2} \cos ^{2}(\theta), \quad \Delta \equiv r^{2}-2 M r+a^{2}+Q^{2}
\end{aligned}
$$

This represents a gravitational field of non-null Einstein-Maxwell type, with corresponding EM potential 1 -form $A_{a}=\operatorname{Qr}\left(a \sin ^{2}(\theta) d_{a} \phi-d_{a} t\right) / \rho^{2}$ (see, e.g., [25]).

Consider the "laboratory" observer with 4-velocity

$$
u^{a}=u_{\mathrm{lab}}^{a} \equiv \frac{\rho}{\sqrt{\Delta_{c}}} \partial_{t}^{a}, \quad \Delta_{c} \equiv \Delta-a^{2} \sin ^{2}(\theta)=r^{2}-2 M r+a^{2} \cos ^{2}(\theta)+Q^{2} .
$$

The natural orthonormal tetrad associated to this observer is $\left(u_{\text {lab }}^{a}, e_{r}^{a}, e_{\theta}^{a}, e_{\phi}^{a}\right)$ with

$$
e_{r}^{a}=\frac{\sqrt{\Delta}}{\rho} \partial_{r}^{a}, \quad e_{\theta}^{a}=\frac{1}{\rho} \partial_{\theta}^{a}, \quad e_{\phi}^{a}=\frac{\sqrt{\Delta_{c} / \Delta}}{\rho \sin (\theta)} \partial_{\phi}^{a}+\frac{a \sin (\theta)\left(Q^{2}-2 M r\right)}{\rho \sqrt{\Delta_{\Delta_{c}}}} \partial_{t}^{a}=\rho \sin (\theta) \sqrt{\frac{\Delta}{\Delta_{c}}} \nabla^{a} \phi
$$


where $\nabla^{a} \phi \equiv g^{a b} d_{b} \phi$ is the gradient of the coordinate $\phi$. The observer measures electric and magnetic fields $E^{a}=F_{b}^{a} u_{\text {lab }}^{b}$ and $B^{a}=\star F_{b}^{a} u_{\text {lab }}^{b}$ given by

$$
\begin{aligned}
E^{a} & =\frac{Q}{\rho^{4} \sqrt{\Delta_{c}}}\left\{\sqrt{\Delta}\left[r^{2}-a^{2} \cos ^{2}(\theta)\right] e_{r}^{a}-a^{2} r \sin (2 \theta) e_{\theta}^{a}\right\}, \\
B^{a} & =\frac{Q a}{\rho^{4} \sqrt{\Delta_{c}}}\left\{2 r \sqrt{\Delta} \cos (\theta) e_{r}^{a}+\left[r^{2}-a^{2} \cos ^{2}(\theta)\right] \sin (\theta) e_{\theta}^{a}\right\} .
\end{aligned}
$$

These can be assembled into the complex vector

$$
f^{a}=E^{a}-i B^{a}=\frac{Q}{(r+i a \sin (\theta))^{2}} x^{a}, \quad x^{a}=\frac{\sqrt{\Delta} e_{r}^{a}-i a \sin (\theta) e_{\theta}^{a}}{\sqrt{\Delta_{c}}},
$$

where $x^{a}$ is a unit vector. From (22) and (27) relevant EM invariants can be calculated:

$$
\sqrt{I_{F}}=\frac{Q}{[r+i a \cos (\theta)]^{2}}, \quad \chi=\frac{\left|I_{F}\right|}{8 \pi}=\frac{Q^{2}}{8 \pi \rho^{4}},
$$

while the EM energy density and Poynting vector relative to $u_{\text {lab }}^{a}$ are

$$
\rho_{\mathrm{em}}=\frac{\Delta+a^{2} \sin ^{2}(\theta)}{\Delta-a^{2} \sin ^{2}(\theta)} \chi, \quad p^{a}=\frac{a Q^{2} \sqrt{\Delta} \sin (\theta)}{4 \pi \rho^{4}\left[\Delta-a^{2} \sin ^{2}(\theta)\right]} e_{\phi}^{a} .
$$

Concerning the Weyl tensor, the electric and magnetic parts relative to $u_{\text {lab }}^{a}$ can be assembled into the complex tensor $Q_{a b}=E_{a b}-i H_{a b}$. In the orthonormal triad $B$ given in 149) the associated operator $\mathrm{Q}$ is represented by the $3 \times 3$ matrix

$$
[\mathrm{Q}]_{B}=\lambda\left[\begin{array}{ccc}
-2-3 a^{2} \sin ^{2}(\theta) / \Delta_{c} & 3 i a \sin (\theta) \sqrt{\Delta} & 0 \\
3 i a \sin (\theta) \sqrt{\Delta} & 1+3 a^{2} \sin ^{2}(\theta) / \Delta_{c} & 0 \\
0 & 0 & 1
\end{array}\right], \quad \lambda=\frac{M[r-i a \cos (\theta)]-Q^{2}}{\rho^{2}[r+i a \cos (\theta)]^{2}}
$$

This satisfies (68), and thus the spacetime is of Petrov type $\mathrm{D}$ (also in the pure Kerr limit case $Q=0$ ) with a double eigenvalue $\lambda$ and simple eigenvalue $-2 \lambda{ }^{14}$ Clearly, $e_{\phi}^{a}$ spans an eigendirection with eigenvalue $\lambda$. The eigendirection corresponding to $-2 \lambda$ is easily found to be spanned by $x^{a}$ given in $(152)$, which is proportional to $f^{a}$ when $Q \neq 0$. By virtue of $(95)$ we have thus found back the well-known fact that the Kerr-Newman spacetime is a Petrov type D doubly aligned non-null Einstein-Maxwell field [19]. Therefore the results of Sec. 5.2.1 apply. By (82) and (155) the proper gravitational super-energy density is given by

$$
\xi=\frac{3 M^{2}}{2 \rho^{8}}\left[a^{2} \cos ^{2}(\theta)+\left(r-Q^{2} / M\right)^{2}\right]
$$

while the super-energy density and super-Poynting vector measured by $u_{\text {lab }}^{a}$ are

$$
\frac{2 \rho_{\mathrm{g}}}{\xi}=3\left[\frac{\Delta+a^{2} \sin ^{2}(\theta)}{\Delta-a^{2} \sin ^{2}(\theta)}\right]^{2}-1, \quad \mathcal{P}^{a}=\frac{9 M^{2} a \sqrt{\Delta}\left[\Delta+a^{2} \sin ^{2}(\theta)\right]\left[a^{2} \cos ^{2}(\theta)+(r-Q / M)^{2}\right]}{2 \rho^{8}\left[\Delta-a^{2} \sin ^{2}(\theta)\right]^{2}} e_{\phi}^{a},
$$

in agreement with (96). Thus the Poynting and super-Poynting vectors are aligned eigenvectors of $\mathrm{Q}$ with eigenvalue $\lambda$ (see remark 5.7), which are moreover orthogonal to the surfaces of constant $\phi$ (i.e., their spatial component is purely azimuthal), just as the Poynting vector (145) in the case of an EM spinning charge in Minkowski spacetime (but the Poynting vectors (145) and (154) die off as $r^{-5}$, whereas the super-Poynting vector (157) dies off as $r^{-7}$ ). On the EM side this means that there is a flow of energy around closed circular loops parallel to the equatorial plane. Motivated by the EM analogy, some authors interpret $\mathcal{P}^{a}$ as representing a flux of "super-energy" [3, 12, 13, 6]; in the spirit of such interpretation, 

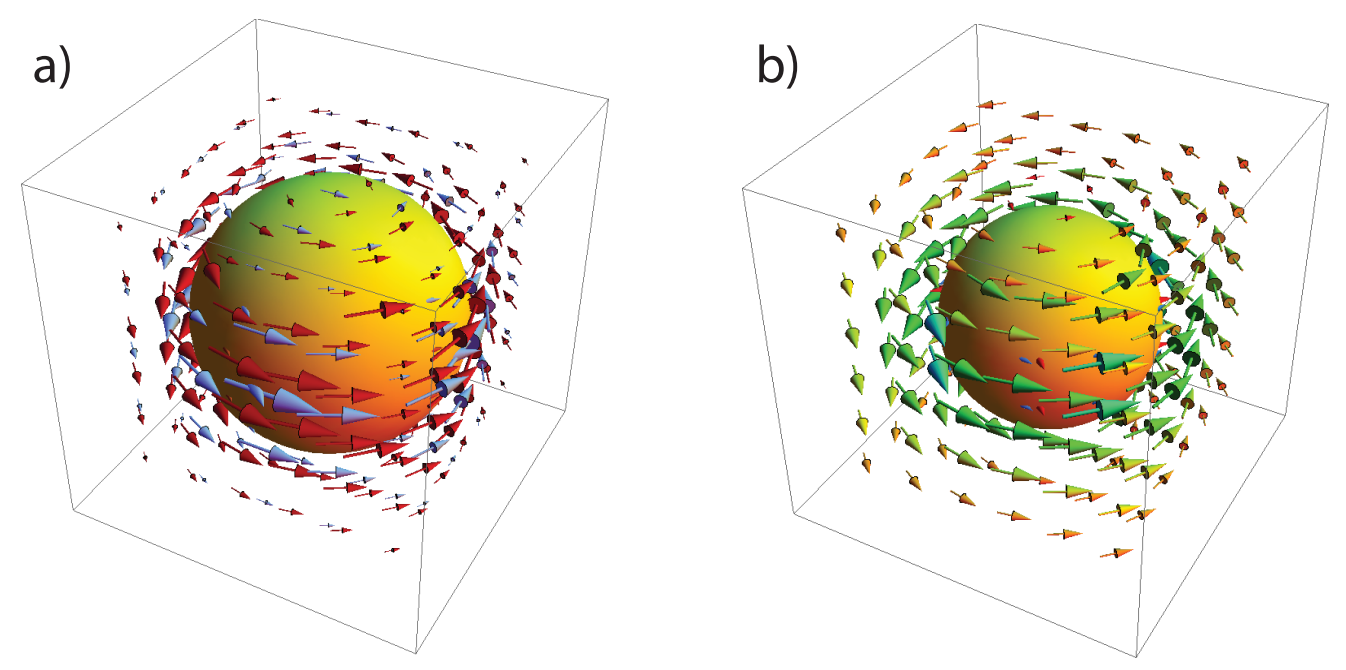

Figure 6: Kerr-Newman spacetime. a) Red arrows: Poynting vector $p^{a}$ as measured by the laboratory observers $u_{\text {lab }}^{a} \propto \partial_{t}^{a}$, signaling a stationary energy flow along circular loops parallel to the equatorial plane; blue arrows: super-Poynting vector $\mathcal{P}^{a}$ as measured by $u_{\text {lab }}^{a}$, suggesting an analogous stationary flow of super-energy. b) The principal observers $u^{\prime a}$ (for which both $p^{\prime a}$ and $\mathcal{P}^{\prime a}$ vanish) move along $p^{a} \propto \mathcal{P}^{a}$ with angular velocity (161), and have an arbitrary velocity along $\mathfrak{e}^{a} \propto \partial_{r}^{a}$. The special case of the canonical principal observers $u^{\prime a}=u_{\Sigma}^{a}$ (Carter observers), in circular motion with relative velocity parallel to $p^{a}$ and $\mathcal{P}^{a}\left[v\left(u^{\prime}, u\right)^{a}=v_{\| p}^{a}=v_{\| \mathcal{P}}^{a}\right]$ is represented.

one would say that in the Kerr and Kerr-Newman spacetime there is a stationary flow of super-energy around closed loops parallel to the equatorial plane; see Fig. 6a.

We want to determine the boosts to the EM and Weyl principal observers. Since $\Sigma=\Sigma_{F}=\Sigma_{C}$ these should coincide. Indeed, from (49)-(50) and (88)- 89$)$ we find $\zeta_{\mathrm{em}}=\zeta_{\mathrm{g}}=1+2 a^{2} \sin ^{2}(\theta) / \Delta_{c}$,

$$
\cosh \left(\psi_{\Sigma_{F}}\right)=\cosh \left(\psi_{\Sigma_{C}}\right)=\sqrt{\Delta / \Delta_{c}}, \quad v_{\| p}^{a}=v_{\| \mathcal{P}}^{a}=\frac{a \sin (\theta)}{\sqrt{\Delta}} e_{\phi}^{a}=: v_{\| e_{\phi}}^{a}
$$

and the expression for the principal observer obtained by projecting $u_{\text {lab }}^{a}$ onto $\Sigma$ :

$$
u_{\Sigma}^{a}=\frac{1}{\rho \sqrt{\Delta}}\left[\left(r^{2}+a^{2}\right) \partial_{t}^{a}+a \partial_{\phi}^{a}\right]
$$

From (58, 294 and 152 we simply have

$$
\mathfrak{e}^{a}=\mathfrak{e}_{F}^{a}=\mathfrak{e}_{C}^{a}=\Re\left(x^{a}\right)=\sqrt{\Delta / \Delta_{c}} e_{r}^{a},
$$

and so according to (57) or 57) the (EM or Weyl) principal observers, which are all $u^{\prime a} \in \Sigma$, move relative to the laboratory observers $u_{\mathrm{lab}}^{a}$ with relative velocity $v\left(u^{\prime}, u_{\mathrm{lab}}\right)^{a}=v_{\| e_{\phi}}^{a}+v_{\| e_{r}}^{a}$, where $v_{\| e_{\phi}}^{a}$ is the fixed component parallel to the (super-)Poynting vector as given in 158) and $v_{\| e_{r}}^{a}$ an arbitrary radial component, and are obtained by arbitrarily boosting $u_{\Sigma}^{a}$ along $\mathfrak{e}^{a} \propto e_{r}^{a}$. They consist thus of all observers $u^{\prime \alpha}$ with angular velocity

$$
\omega_{\left(\mathcal{P}^{\prime}=0\right)}=\omega_{\left(p^{\prime}=0\right)}=\frac{u^{\prime \phi}}{u^{\prime 0}}=\frac{a}{\left(a^{2}+r^{2}\right)},
$$

\footnotetext{
${ }^{14}$ The exceptional spacetime points with $\lambda=0 \Leftrightarrow C_{a b c d}=0$ are given by $\left\{\theta=\frac{\pi}{2}, r=Q^{2} / M\right\}$; they lie on the equatorial plane and, with $a \geq 0$, outside the event horizon precisely when $a / M \leq(Q / M)^{2}-(Q / M)^{4}$.
} 
and with an arbitrary radial component $u^{\prime r}$. The special case $u^{\prime r}=0$ (that is, $u^{\prime a}=u_{\Sigma}^{a}$ ) corresponds to a congruence of principal observers in circular motion, called the Carter observers [26, 55, which are plotted in Fig. 6b. At each point the Carter observer is the one that realizes both the EM Wheeler result (theorem 4.3) and the gravitational Wheeler analogue (theorem 5.5), i.e., it is the principal observer that moves, with respect to $u_{\text {lab }}^{a}$, in the direction of $p^{a} \propto \mathcal{P}^{a}$. Comparing with the principal observers of the spinning charged body in flat spacetime illustrated in Fig. 5, and assuming a uniform charge and mass distribution so that $\mu=(Q / 2 M) J=Q a / 2$, we have that, asymptotically, the angular velocities 146) and (161) match up to a factor $2{ }^{15}$ In this context we note that in the work by Rosquist [56 it was shown that the Kerr-Newman metrics can be obtained by a re-scaling of an orthonormal tetrad field in Minkowski spacetime, constructed from spheroidal coordinates in differential rotation, each spheroidal shell $r=$ constant rotating rigidly. It turns out that the angular velocity of the shells is precisely $\omega_{\left(\mathcal{P}^{\prime}=0\right)}$; therefore, the principal observers are precisely those comoving with Rosquist's shells, plus those moving radially with respect to them. Finally, we note that taking the limit $M \rightarrow 0$ in (147) one obtains an electromagnetic field in the flat Minkowski metric written in spheroidal coordinates (cf. footnote 15), whose principal observers retain the same properties (such field having, however, a complicated source whose form is not enlightening, see e.g. [57] and references therein).

\subsection{Kasner spacetimes}

Consider the Kasner vacuum spacetimes, which are spatially homogeneous of Bianchi type I. In suitable coordinates they admit the diagonal line element [19]

$$
d s^{2}=-d t^{2}+t^{2 f_{1}}\left(d x^{1}\right)^{2}+t^{2 f_{2}}\left(d x^{2}\right)^{2}+t^{2 f_{3}}\left(d x^{3}\right)^{2},
$$

where $\left(f_{1}, f_{2}, f_{3}\right) \in \mathbb{R}^{3}$ is a parameter triple satisfying $\sum_{i=1}^{3} f_{i}=\sum_{i=1}^{3} f_{i}^{2}=1$. The Weyl eigenvalues are $\lambda_{i}=-f_{j} f_{k} / t^{2}$, so the Weyl tensor is purely electric at each point, implying $q=0$ (see remark 5.3 ). By permuting the coordinates we may assume $f_{3} \leq f_{2} \leq f_{1}$. The metric (162) represents Minkowski spacetime for $f_{3}=f_{2}=0, f_{1}=1$, and a Petrov type D plane symmetric vacuum for $f_{3}=-1 / 3, f_{2}=$ $f_{1}=2 / 3$. On excluding these cases henceforth the Petrov type is $\mathrm{I}\left(M^{+}\right)$, with $-1 / 3<f_{3}<0<-f_{3}<$ $f_{2}<2 / 3<f_{1}<1$ and $\left|f_{3}\right|<\left|f_{2}\right|<\left|f_{1}\right|{ }^{16}$ such that $0<\left|\lambda_{1}\right|<\left|\lambda_{2}\right|<\left|\lambda_{3}\right|$.

The essentially unique principal tetrad vectors lie along the coordinate vector fields at each point: $e_{0}^{a}=\partial_{t}^{a}$ and $e_{i}^{a}=\partial_{x^{i}}^{a} / t^{f_{i}}$. Observers of the type 132), moving relative to $e_{0}^{a}$ along one of the $e_{i}^{a}$, measure, by 133 , a super-Poynting vector

$$
\mathcal{P}^{a}=-\frac{1}{8} t^{-4} f_{i}^{2}\left(f_{j}-f_{k}\right)^{2} \sinh (4 \varphi)\left[\sinh (\varphi) e_{0}^{a}+\cosh (\varphi) e_{i}^{a}\right] \propto v^{a}\left(e_{0}, u\right),
$$

i.e., along the direction of $v\left(e_{0}, u\right)^{a}$; hence, their boost back to the principal observer $e_{0}^{a}$ is along $\mathcal{P}^{a}$, see the left panel of Fig. 3. The other non-principal observers $u^{a}$ for which $v\left(e_{0}, u\right)^{a} \| \mathcal{P}^{a}$ have $u^{1}=0$ and relative velocities parallel to $u^{2} e_{2}^{a}+u^{3} e_{3}^{a}$ with $u^{2}=\left[2\left(1-f_{1} / f_{3}\right) / 3\right]^{-1 / 2} \sinh (\varphi), u^{3}= \pm\left[2\left(f_{1} / f_{2}-\right.\right.$ $1) / 3]^{-1 / 2} \cosh (\varphi)$, see example 5.12 and the right panel of Fig 3

Take now an observer moving along the bisector of $e_{1}^{a}$ and $e_{2}^{a}: u^{\prime a}=\cosh (\varphi) e_{0}^{a}+\sinh (\varphi) e_{12}^{a}$, where $e_{12}^{2}=\left(e_{1}^{a}+e_{2}^{a}\right) / \sqrt{2}$. Then the vector defined in 140$)$ is

$$
D^{a}=\frac{3}{16} t^{-4} f_{1} f_{2} f_{3}\left(f_{1}-f_{2}\right) \sinh (2 \varphi)^{2} e_{3}^{a},
$$

which is non-zero when the Petrov type is $\mathrm{I}\left(M^{+}\right)$, again in accordance with theorem $5.10{ }^{17}$ hence we have $v\left(e_{0}, u^{\prime}\right)^{a} \nVdash \mathcal{P}^{\prime a}$ (i.e., the boost that takes $u^{a}$ into the principal observer $e_{0}^{a}$ is not along $\mathcal{P}^{\prime a}$, as in the left panel of Fig. 3).

\footnotetext{
${ }^{15}$ In the equatorial plane $\theta=\theta=\pi / 2$ such matching (up to the factor 2) is actually exact since the metric (147) is written in spheroidal coordinates which, in the limit $M \rightarrow 0$, are related to the spherical coordinates of Sec. 6.1 by Eq. (28) of [56].

${ }^{16}$ This can be readily inferred from $\sum_{i=1}^{3} f_{i}=\sum_{i=1}^{3} f_{i}^{2}=1$, or from the parametrization $f_{i}=\frac{1}{3}[1+2 \cos (\psi+(1-i) 2 \pi / 3)]$, $0<\psi<\pi / 3$, which is compatible with $f_{3} \leq f_{2} \leq f_{1}$.

${ }^{17}$ As expected, $D^{a}=0$ when the spacetime is Minkowski $\left(f_{3}=f_{2}=0, f_{1}=1\right)$ or type $\mathrm{D}\left(f_{3}=-1 / 3, f_{2}=f_{1}=2 / 3\right)$.
} 
Given the electric and magnetic parts of the Weyl tensor as measured by any observer $u^{a}$ one can easily apply the algorithm at the end of Sec. 5.3 . one readily finds the eigenvalues $\lambda_{i}=-f_{j} f_{k} / t^{2}$ of the corresponding endomorphism Q, calculates the unit eigenvectors $x_{i}^{a}$ by $(112)-(113)$, and finds the Weyl principal tetrad from 109)-114.

\section{Summary and discussion}

In electromagnetism (EM) and general relativity theory, principal observers are defined to be those observers for which the (super-)Poynting vector associated to the Faraday, resp., the Weyl tensor vanishes. This precisely happens in the EM case when the electric and magnetic fields are aligned, and in the gravitational case when the electric and magnetic parts of the Weyl tensor commute. Another criterion is that the (super-)energy density attains minimum value. On identifying an observer at a point with its 4-velocity vector, the instantaneous existence and number of principal observers simply depends on the algebraic type of the Faraday or Weyl tensor at the point. Principal observers precisely exist when the relevant tensor is of 'diagonal' type. In the (generic) non-null EM case and the (non-generic) Petrov type D gravitational case the principal observers' velocities are the unit, future-pointing timelike vectors of a distinguished 2-plane, viz. the timelike principal plane, which induces a natural structure (observer geometry) on the set of observers at the point. In the (generic) Petrov type I gravitational case there is a unique principal observer.

We have focused on the relation of general observers to principal ones, employing a novel calculus on the complexified rest space of a general observer. In a non-null EM field the 'EM Wheeler result' holds: given an arbitrary observer $u^{a}$, measuring a Poynting vector $p^{a}$, a principal observer $u_{\mathrm{em}}^{a}$ can always be reached through a boost in the direction of $p^{a}$. We have clarified the underlying geometric nature of this result by showing that $u_{\mathrm{em}}^{a}$ is nothing but the orthogonal projection $u_{\Sigma}^{a}$ of $u^{a}$ onto the timelike principal plane. As our main achievement we have demonstrated that the 'gravitational Wheeler analogue' (obtained by changing Poynting to super-Poynting in the statement of the EM Wheeler result) holds in a Petrov type D gravitational field, giving a deduction that emphasized the mathematical analogy between the two situations. Moreover, the (super-)energy density depends on and increases with the rapidity relative to $u_{\Sigma}^{a}$. Hence the observer geometries in these two cases are simply linked to (super-)Poynting vectors and (super-)energy densities measured by observers. This simplicity is not shared by the Petrov type I gravitational case, where the level sets of the super-energy density are rather complicated, and we proved that only for a one-dimensional variety of observers the (unique) principal observer lies in the plane spanned by the observer and the relative super-Poynting vector.

In all three cases (non-null EM, and Petrov type D and I gravitational cases) we have outlined algorithms to compute all principal observers from the electric and magnetic fields or electric and magnetic parts of the Weyl tensor as measured by an arbitrary observer.

As a final note, we briefly comment on the (unavoidable) question of the physical significance of the super-Poynting vector. Because it is a flux of super-energy, its physical meaning, similarly to that of the super-energy itself, remains unclear; a solid connection to observable effects has yet to be established. It is tempting however to draw a parallelism with the situation for the Poynting vector $p^{a}$, prior to general relativity. Although $p^{a}$ was, in some dynamical situations (such as electromagnetic radiation), known to be a physically measurable quantity (e.g. by the momentum imparted on a mirror), the Poynting vector in stationary settings such as the spinning charge in Fig. 5 , indicating a flow of energy circulating around in closed loops, has been questioned due to its strangeness 18 and often dismissed as immaterial, since no observable consequences were known [58. General relativity changed the picture, in that all forms of energy and energy currents act as sources for the gravitational field, and are therefore, in theory, measurable. In particular, $p^{a}$ should be measurable through the frame-dragging effect it generates [59, 60,

\footnotetext{
${ }^{18}$ Indeed $p^{a}$ looks very strange sometimes. Another example is the case of a wire carrying a current, where outside the wire $p^{a}$ is orthogonal to the wire, pointing inwards. That implies that the electric energy does not reach the electrons by flowing through the wire, but instead by going from the battery into a wide area of space around, and then inward to the wire [58].
} 
61. It should also (being an energy current) generate a "Magnus"-like force on spinning test bodies 62. The super-Poynting vector is likewise believed to accompany gravitational radiation [11, 12, 13, 14, 15, 16, and, in stationary settings such as those in Fig. 6, where (similarly to its EM counterpart) it circulates around in closed loops, observable consequences have been sought, namely an attempted link to framedragging 63 .

\section{Acknowledgements}

We thank J. M. M. Senovilla for useful comments and remarks. L.W. was supported through a BOF research grant of Ghent University and through the Research Council of Norway, Toppforsk grant no. 250367: Pseudo- Riemannian Geometry and Polynomial Curvature Invariants: Classification, Characterisation and Applications. L.F.C. and J.N. were supported by FCT/Portugal through projects UID/MAT/04459/2019 and UIDB/MAT/04459/2020, and grant SFRH/BDP/85664/2012.

\section{A Orthonormal frames in self-dual bivector and tangent space}

Three unitary, orthogonal self-dual bivectors $\mathcal{X}_{a b}^{i}$ form an orthonormal frame of $S^{+}\left[-\frac{1}{4}\left(\mathcal{X}^{m}\right)^{a b} \mathcal{X}_{a b}^{n}=\delta_{m n}\right.$, $m, n=1,2,3]$ which is moreover oriented if $\mathcal{X}_{a b}^{i}=-i \mathcal{X}_{a c}^{j}\left(\mathcal{X}^{k}\right)^{c}{ }_{b}$. The relation

$$
\mathcal{X}_{a b}^{i}=\left[2\left(e_{0}\right)_{[a}\left(e_{i}\right)_{b]}\right]^{\dagger}=2\left(e_{0}\right)_{[a}\left(e_{i}\right)_{b]}+2 i\left(e_{j}\right)_{[a}\left(e_{k}\right)_{b]}, \quad i=1,2,3
$$

determines a bijection between restricted orthonormal tetrads $\left(e_{0}^{a}, e_{i}^{a}\right)$ of $T_{p} M$ and oriented orthonormal frames $\left(\mathcal{X}_{a b}^{i}\right)$ of $S^{+}$. This can be seen as a consequence of the group isomorphism between the proper orthochronous Lorentz group and the group of proper orthogonal transformations of $S^{+}$, see for instance [19] or [53. Here we present a simple and direct geometric proof; cf. [65, pp. 246-248]. It is easy to verify that the triple $\left(\mathcal{X}_{a b}^{i}\right)$ constructed as in 163 from a restricted orthonormal tetrad $\left(e_{0}^{a}, e_{i}^{a}\right)$ is an oriented orthonormal frame of $S^{+}$. Conversely, we need to show that any oriented orthonormal frame $\left(\mathcal{X}_{a b}^{i}\right)$ of $S^{+}$ can be written as in 163), where $\left(e_{0}^{a}, e_{i}^{a}\right)$ is moreover uniquely determined. By 9 and $\mathcal{X}_{a b}^{i}=X_{a b}^{i}-i \star X_{a b}^{i}$ the orthonormality condition $-\frac{1}{4}\left(\mathcal{X}^{m}\right)^{a b} \mathcal{X}_{a b}^{n}=\delta_{m n}$ is equivalent to the three sets of conditions

$$
I_{X_{i}}=1, \quad i=1,2,3, \quad\left(\star X^{i}\right)^{a b} X_{a b}^{j}=0, \quad i \neq j, \quad\left(X^{i}\right)^{a b} X_{a b}^{j}=-\left(\star X^{i}\right)^{a b} \star X_{a b}^{j}=0, \quad i \neq j .
$$

The first set implies that $\left(X^{i}\right)^{a b}=2 r_{i}^{[a} s_{i}^{b]},\left(\star X^{i}\right)^{a b}=2{r^{\prime}}_{i}^{[a}{s^{\prime}}_{i}^{b]}$ for each $i$ separately, where the $\left(r_{i}^{a}, s_{i}^{a}, r_{i}^{\prime a},{s^{\prime}}_{i}^{a}\right)$ are restricted orthonormal frames. For $i=1,2,3$ the blades of $X_{a b}^{i}$ and $\star X_{a b}^{i}$ are denoted by $\Sigma_{i}$ and $\Sigma_{i}^{\perp}$, respectively. Consider now a fixed $i$. For each $j \neq i$ the second (third) set of [164 yields $\epsilon_{a b c d} r_{i}^{a} s_{i}^{b} r_{j}^{c} s_{j}^{d}=0$ $\left(\epsilon_{a b c d} r_{i}^{a} s_{i}^{b} r_{j}^{\prime c} s_{j}^{\prime d}=0\right)$, which means that $\Sigma_{i}$ and $\Sigma_{j}\left(\Sigma_{j}^{\perp}\right)$ together span a 3 -dimensional subspace of tangent space, and thus intersect in a line $l_{i j}\left(l_{i j}^{\prime}\right)$; here $l_{i j}^{\prime}$ is spacelike since the blade $\Sigma_{j}^{\perp}$ is spacelike, and thus the line $l_{i j}$ is timelike since together with $l_{i j}^{\prime}$ it spans the timelike blade $\Sigma_{i}$. Applying this to $i=2, j=1$ and writing $e_{0}^{a}$ for the unique future-pointing unit tangent vector along $l_{21}$ we infer $\left(X^{1}\right)^{a b}=2 e_{0}^{[a} e_{1}^{b]}$ and $\left(X^{2}\right)^{a b}=2 e_{0}^{[a} e_{2}^{b]}$, where $e_{1}^{a}$ and $e_{2}^{a}$ are uniquely defined unit spacelike vectors which are both orthogonal to $e_{0}^{a}$ and also mutually orthogonal by $\left(X^{2}\right)^{a b} X_{a b}^{1}=0$. Hence we obtain a uniquely defined restricted orthonormal tetrad $\left(e_{0}^{a}, e_{1}^{a}, e_{2}^{a}, e_{3}^{a}\right)$. Next consider $i=3$ and $j=1,2$. Since the timelike lines $l_{3 j}$ belong to $\Sigma_{j}$ they have unit tangent vectors $u_{j}^{a}=\alpha_{j} e_{0}^{a}+\beta_{j} e_{j}^{a}, j=1,2$, where $\alpha_{j}, \beta_{j} \in \mathbb{R}$ and $\alpha_{1} \alpha_{2} \neq 0$. Assume $\left(\beta_{1}, \beta_{2}\right) \neq(0,0)$; then the lines $l_{31}$ and $l_{32}$ would not coincide and thus would span $\Sigma_{3}$; hence $\left(X^{3}\right)^{a b}=C u_{1}^{[a} u_{2}^{b]}$ for certain $C \neq 0$, but then $\left(X^{3}\right)^{a b} X_{a b}^{j}=0, j=1,2$ gives $\beta_{1} \alpha_{2}=\beta_{2} \alpha_{1}=0$ and thus the contradiction $\alpha_{1} \alpha_{2}=0$. Hence $\beta_{1}=\beta_{2}=0$ and $l_{31}=l_{32}=l_{21}$, implying $\left(X^{3}\right)^{a b}=2 e_{0}{ }^{[a} \nu^{b]}$ with

\footnotetext{
${ }^{19}$ Such connection is however not well established. A known counter-example 64 is the Gödel universe, where a rigid frame exists relative to which $\mathcal{P}^{\alpha}$ vanishes everywhere, but still the "gravitomagnetic field" $H^{a}=2 \omega^{a}\left(\omega^{a} \equiv\right.$ vorticity), and thus frame-dragging, is present [37. This shows that $\mathcal{P}^{a}$ is (at best) not the sole source of frame-dragging. It should be noticed in this context that $\mathcal{P}^{a}$ is built on tidal fields (namely $H_{a b}$ ), whereas frame-dragging is directly related instead to $H^{a}$, and it is important to distinguish between the two (see [37]).
} 


\begin{tabular}{c|cccccc} 
Petrov type & $\mathrm{I}$ & $\mathrm{II}$ & $\mathrm{III}$ & $\mathrm{D}$ & $\mathrm{N}$ & 0 \\
\hline$d$ & 3 & 3 & 3 & 2 & 2 & 1 \\
$N$ & 3 & 2 & 1 & 2 & 1 & 1
\end{tabular}

Table 1: Petrov types: $d \equiv$ degree of $m(x), N \equiv$ number of different eigenvalues of $\mathrm{C}$ or $\mathrm{Q}$.

$\nu^{a}$ unit spacelike and orthogonal to $e_{0}^{a}$. By $\left(X^{3}\right)^{a b} X_{a b}^{j}=0, j=1,2$, it follows that $\nu^{a}$ is also orthogonal to $e_{1}^{a}$ and $e_{2}^{a}$, and thus equals $\pm e_{3}^{a}$. If we moreover require the orthonormal frame $\left(\mathcal{X}_{a b}^{i}\right)$ to be oriented by $\mathcal{X}_{a b}^{i}=-i \mathcal{X}_{a c}^{j}\left(\mathcal{X}^{k}\right)^{c}{ }_{b}$, then $\nu^{a}=e_{3}^{a}$ and we arrive at $\left(\mathcal{X}^{i}\right)^{a b}=\left(2 e_{0}^{[a} e_{i}^{b]}\right)^{\dagger}$, which concludes the proof.

Geometrically, $e_{0}^{a}$ is thus the observer along the intersection of the blades $\Sigma_{i}$ of the simple 2-forms $X_{a b}^{i}, i=1,2,3$, while for fixed $i, e_{i}^{a}=\left(X^{i}\right)^{a}{ }_{b} e_{0}^{b}=\left(\mathcal{X}^{i}\right)^{a}{ }_{b} e_{0}^{b}$ lies along the joint intersection of $\Sigma_{i}, \Sigma_{j}^{\perp}, \Sigma_{k}^{\perp}$.

\section{B Notes on the Weyl and Bel-Robinson tensors}

\section{B.1 Petrov classification of the Weyl tensor in terms of $Q^{a}{ }_{b}$}

The Petrov classification of the Weyl tensor into the six Petrov types can be formulated in terms of the complex tensor $Q^{a}{ }_{b} \equiv \mathcal{C}^{a}{ }_{c b d} u^{c} u^{d}=$ relative to any observer $u^{a}$, the projector $h_{b}^{a} \equiv \delta_{b}^{a}+u^{a} u_{b}$ onto the observer's complexified rest space $\mathbb{C} u^{\perp}$, and the complex invariants $I=Q^{a}{ }_{b} Q^{b}{ }_{a}$ and $J=Q^{a}{ }_{b} Q^{b}{ }_{c} Q^{c}{ }_{a}$ :

- Petrov type I: $I^{3} \neq 6 J^{2}$;

- Petrov type II: $I^{3}=6 J^{2} \neq 0,\left(Q^{a}{ }_{c}-\lambda h_{c}^{a}\right)\left(Q^{c}{ }_{b}+2 \lambda h_{b}^{c}\right) \neq 0, \lambda \equiv-J / I$;

- Petrov type D: $I^{3}=6 J^{2} \neq 0,\left(Q^{a}{ }_{c}-\lambda h_{c}^{a}\right)\left(Q^{c}{ }_{b}+2 \lambda h_{b}^{a}\right)=0, \lambda \equiv-J / I$;

- Petrov type III: $Q^{a}{ }_{c} Q^{c}{ }_{d} Q^{d}{ }_{b}=0 \neq Q^{a}{ }_{c} Q^{c}{ }_{b}$;

- Petrov type N: $Q^{a}{ }_{c} Q^{c}{ }_{b}=0 \neq Q_{b}^{a}$;

- Petrov type O: $Q^{a}{ }_{b}=0$.

Regarding the Petrov type D case, $\left(Q^{a}{ }_{c}-\lambda h_{c}^{a}\right)\left(Q^{c}{ }_{b}+2 \lambda h_{b}^{a}\right)=0$ with $\lambda \neq 0$ actually implies $I=6 \lambda^{2}, J=$ $-6 \lambda^{3}$ and thus $I^{3}=6 J^{2}$ and $\lambda=-J / I$. By (63) the endomorphism $Q: \nu^{a} \mapsto Q^{a}{ }_{b} \nu^{b}$ of $\mathbb{C} u^{\perp}$ (for any $u^{a}$ ) has the same characteristic polynomial

$$
c(x)=\prod_{k=1}^{3}\left(x-\lambda_{k}\right)=x^{3}-\frac{1}{2} I x-\frac{1}{3} J
$$

and the same minimal polynomial polynomial $m(x)$ as the endomorphism $\mathrm{C}: \mathcal{X}_{a b} \mapsto-\frac{1}{4} \mathcal{C}_{a b}{ }^{c d} \mathcal{X}_{c d}$ of $S^{+}$. The roots $\lambda_{k}$ of the cubic $c(x)$ are the eigenvalues of these endomorphisms. The Petrov types are characterized by the degree $d$ of $m(x)$ and the number $N$ of different eigenvalues, as summarized in Table 1. The endomporphisms $\mathrm{C}$ and $\mathrm{Q}$ are diagonalizable iff $N=d$, i.e. iff the Petrov type is $\mathrm{I}, \mathrm{D}$ or $\mathrm{O}$. Petrov types III, N and 0 have $\lambda=0$ as their only triple eigenvalue; Petrov types II and D have a double eigenvalue $\lambda=-J / I$ and a simple eigenvalue $-2 \lambda$; the three simple eigenvalues for Petrov type I can be calculated by Cardano's formula:

$$
\lambda_{k}=B^{\frac{1}{3}} e^{\frac{2(k-1) \pi i}{3}}+\frac{I}{6} B^{-\frac{1}{3}} e^{-\frac{2(k-1) \pi i}{3}}, \quad B \equiv \frac{J}{6}+\frac{1}{6} \sqrt{\frac{-D}{6}}, \quad D \equiv I^{3}-6 J^{2}, \quad k=1,2,3 .
$$

Here one can take any choice for the (complex) cube and square roots, provided that in the subcase $I=0$ one chooses $\sqrt{J^{2}}=+J$, such that always $B \neq 0$. 


\section{B.2 Bel-Robinson endomorphism and degenerate Petrov type I}

The Bel-Robinson tensor $T^{a b}{ }_{c d}$ defines an endomorphism on the $9 \mathrm{~d}$ vector space of trace-free symmetric tensors; it has eigenvalues $t_{i}, \tau_{i}, \bar{\tau}_{i}, i=1,2,3$ which can be parametrized by $t_{i}$ or $p_{i} \equiv \Re\left(\tau_{i}\right)$ [38, 43, 13]:

$$
\begin{aligned}
& \tau_{i} \equiv \lambda_{j} \bar{\lambda}_{k} \equiv p_{i}+i q, \quad t_{i} \equiv\left|\lambda_{i}\right|^{2}=-\left(p_{i}+p_{j}\right) \quad \Leftrightarrow \quad p_{i}=\frac{1}{2}\left(t_{i}-t_{j}-t_{k}\right), \\
& q^{2}=p_{2} p_{3}+p_{3} p_{1}+p_{1} p_{2}=\frac{1}{2}\left(t_{2} t_{3}+t_{3} t_{1}+t_{1} t_{2}\right)-\frac{1}{4}\left(t_{1}^{2}+t_{2}^{2}+t_{3}^{2}\right) .
\end{aligned}
$$

Alternatively, one can use the invariants $r_{i} \equiv\left|\lambda_{j}-\lambda_{k}\right|^{2}, i=1,2,3$, coinding with (C.6) in [13]:

$$
\begin{aligned}
r_{i} & =t_{j}+t_{k}-2 p_{i}=2\left(t_{j}+t_{k}\right)-t_{i}=-\left(4 p_{i}+p_{j}+p_{k}\right) \\
\Leftrightarrow \quad t_{i} & =\frac{1}{9}\left(2 r_{j}+2 r_{k}-r_{i}\right) \quad \Leftrightarrow \quad p_{i}=\frac{1}{36}\left(r_{j}+r_{k}-5 r_{i}\right) .
\end{aligned}
$$

Combining (168) with 170 gives 117$)$. Also note that

$$
t_{j}-t_{k}=p_{j}-p_{k}=\left(r_{j}-r_{k}\right) / 3
$$

By (64) and (167), 168, 171) one obtains

$$
|I|^{2}=\sum_{j=1}^{3} \lambda_{j}^{2} \sum_{k=1}^{3} \bar{\lambda}_{k}^{2}=\sum_{1 \leq j, k \leq 3}\left(\lambda_{j} \bar{\lambda}_{k}\right)^{2}=\sum_{i=1}^{3}\left(t_{i}^{2}+\tau_{i}^{2}+\bar{\tau}_{i}^{2}\right)=4 \sum_{i=1}^{3} p_{i}^{2}-4 q^{2}=2 \sum_{(i j k)}\left(t_{j}-t_{k}\right)^{2} .
$$

The middle expression confirms $|I|^{2}=T^{a b}{ }_{c d} T^{c d}{ }_{a b} \equiv 4 \alpha^{2}$ [38, while the penultimate expression and $16 \xi^{2}=\left(\sum_{i=1}^{3} t_{i}\right)^{2}=4 \sum_{i=1}^{3} p_{i}^{2}+8 q^{2}$ yield the identity (71). From (167), (171), (172) it follows that

$$
I=0 \neq J \quad \Leftrightarrow \quad\left|\lambda_{1}\right|=\left|\lambda_{2}\right|=\left|\lambda_{3}\right| \neq 0 \quad \Leftrightarrow \quad r_{1}=r_{2}=r_{3} \neq 0,
$$

which is the Petrov type I subcase with eigenvalues $\lambda_{k}=e^{\frac{i 2 \pi k}{3}} \alpha, \alpha \in \mathbb{R}$ and $q=3 t_{1}^{2} / 2=r_{1}^{2} / 12 \neq 0$.

The eigenvalue degeneracy of the Bel-Robinson endomorphism was studied in [38] and easily follows from (167). Clearly, all eigenvalues are zero if the Petrov type is $\mathrm{O}, \mathrm{N}$ or III (where $I=J=0$ and all $\left.\lambda_{i}=0\right)$. Excluding these cases, degeneracy occurs precisely when $q=0$ or $t_{j}=t_{k}$ for some $j \neq k$, say $t_{2}=t_{3} \Leftrightarrow\left|\lambda_{2}\right|=\left|\lambda_{3}\right| \Leftrightarrow r_{2}=r_{3}$. By 168-170 the intersection gives two cases:

$$
\begin{aligned}
I^{3}=6 J^{2} \neq 0: & \lambda_{1}=-2 \lambda_{2}=-2 \lambda_{3} \neq 0 \quad \Leftrightarrow \quad t_{1}=4 t_{2}=4 t_{3} \neq 0 \quad \Leftrightarrow \quad r_{1}=0 ; \\
J=0 \neq I: & \lambda_{1}=0 \neq \lambda_{2}=-\lambda_{3} \quad \Leftrightarrow \quad t_{1}=0 \quad \Leftrightarrow \quad r_{1}=4 r_{2}=4 r_{3} \neq 0,
\end{aligned}
$$

where the first covers types II and D and the last is the Petrov type I subcase with a vanishing Weyl eigenvalue, see (66). Consider the dimensionless invariant

$$
M \equiv I^{3} / J^{2}-6 \in \mathbb{C} \cup\{\infty\}
$$

The cases 173, 174, 175 respectively correspond to $M=-6,0, \infty$ and are precisely those cases with only three different Bel-Robinson eigenvalues, where other degenerate cases have six different eigenvalues. For $J \neq 0, M$ can be written as 45 .

$$
M=\frac{2}{9} \mu^{2}, \quad \mu=\frac{\left(\lambda_{3}-\lambda_{2}\right)\left(\lambda_{1}-\lambda_{3}\right)\left(\lambda_{2}-\lambda_{1}\right)}{\lambda_{1} \lambda_{2} \lambda_{3}} .
$$

Multiplying numerator and denominator of $\mu$ with $\bar{\lambda}_{1} \bar{\lambda}_{2} \bar{\lambda}_{3}$ and using (167)-(168) we obtain

$$
\mu=\frac{\left(\tau_{2}-\bar{\tau}_{3}\right)\left(\tau_{3}-\bar{\tau}_{1}\right)\left(\tau_{1}-\bar{\tau}_{2}\right)}{t_{1} t_{2} t_{3}}=\frac{\left(t_{2}-t_{3}\right)\left(t_{3}-t_{1}\right)\left(t_{1}-t_{2}\right)}{t_{1} t_{2} t_{3}}-i \frac{q\left[4 q^{2}+\left(t_{1}+t_{2}+t_{3}\right)^{2}\right]}{2 t_{1} t_{2} t_{3}}
$$


Hence $\mu$ is real (purely imaginary) precisely when $q=0\left(t_{j}=t_{k}\right.$ for some $j$ and $\left.k\right)$; since $q=\Im\left(\lambda_{j} \bar{\lambda}_{k}\right)=$ $\Im\left(\lambda_{j} / \lambda_{k}\right)$ whenever $\lambda_{k} \neq 0$ this proves, in a straighforward way, the known result that $M$ is real nonnegative (non-positive) precisely when the ratio of any two non-zero Weyl eigenvalues is real (there are at least two Weyl eigenvalues with equal moduli). It follows that degeneracy of the Bel-Robinson eigenvalues occurs precisely when $M$ is real or infinite.

For Petrov type $\mathrm{I}(M \neq 0)$ the corresponding cases have been denoted by $\mathrm{I}\left(M^{+}\right), \mathrm{I}\left(M^{-}\right)$and $\mathrm{I}\left(M^{\infty}\right)$ in the extended Petrov classification of the Weyl tensor by Arianrhod and McIntosh 27. They considered the Penrose-Rindler disphenoid on the sphere of null directions, the vertices of which represent the four simple Weyl PNDs and which has 3 pairs of opposite edges with equal lengths $a_{1}, a_{2}, a_{3}$ [65, pp. 249-252], and found that there is degeneracy of two kinds precisely when $M$ is real or infinite:

- one has $a_{j}=a_{k} \Leftrightarrow t_{j}=t_{k}$ and thus there are four edges of equal length if the extended Petrov type is $\mathrm{I}\left(M^{\infty}\right)$ or $\mathrm{I}\left(M^{-}\right)$with $M \neq-6$, and all edges are equal if $M=-6 \Leftrightarrow I=0$;

- the disphenoid is planar, i.e., the PNDs span a $3 \mathrm{~d}$ vector space precisely when $q=0$, corresponding to types $\mathrm{I}\left(M^{+}\right)$and $\mathrm{I}\left(M^{\infty}\right)[66$.

Consider the case $q \equiv \Im\left(\lambda_{j} \bar{\lambda}_{k}\right)=0$, which is thus characterized by the existence of $\sigma \in[0, \pi[$ and real numbers $\lambda_{k}^{\prime}$ (summing to zero) such that $\lambda_{k}=e^{i \sigma} \lambda_{k}^{\prime}, k=1,2,3$. Then

$$
I=e^{2 i \sigma}|I|, \quad J=e^{3 i \sigma} J^{\prime}, J^{\prime}=3 \lambda_{1}^{\prime} \lambda_{2}^{\prime} \lambda_{3}^{\prime} \in \mathbb{R}, \quad \lambda_{k}=\sqrt{I /|I|} \lambda_{k}^{\prime}, \quad M+6=\frac{|I|^{3}}{J^{\prime 2}},
$$

and rewriting 165 in terms of $\lambda_{k}^{\prime},|I|, J^{\prime}$ results in a trigonometric alternative to the expressions 166:

$$
\lambda_{k}=\sqrt{\frac{2 I}{3}} \cos \left(\frac{\arccos L_{\sigma}}{3}-k \frac{2 \pi}{3}\right), \quad L_{\sigma}=\frac{\operatorname{sgn}\left(e^{-3 i \sigma} J\right)}{\sqrt{1+M / 6}}, \quad 2 \sigma=\arg (I) \in[0,2 \pi[, \quad k=1,2,3 .
$$

Since the $\lambda_{k}^{\prime}$ sum to zero there is a unique $\lambda_{k_{0}}^{\prime}$ with smallest absolute value, where if $\left|\lambda_{1}^{\prime}\right|<\left|\lambda_{2}^{\prime}\right| \leq\left|\lambda_{3}^{\prime}\right|$ then either $\lambda_{2}^{\prime}<\lambda_{1}^{\prime} \leq 0<\lambda_{3}^{\prime}$ or $\lambda_{3}^{\prime}<0 \leq \lambda_{1}^{\prime}<\lambda_{2}^{\prime}$. Using $\left|\lambda_{k}\right|=\left|\lambda_{k}^{\prime}\right|$ and $\sqrt{r_{k}}=\left|\lambda_{i}-\lambda_{j}\right|=\left|\lambda_{i}^{\prime}-\lambda_{j}^{\prime}\right|$, and invoking theorem 2 of [44], we obtain:

Proposition B.1. If the Petrov type is I and $q=0$ then there is a unique Weyl eigenvalue with smallest modulus, say $\lambda_{1}$, and the $3 d$ subspace of $T_{p} M$ spanned by the four Weyl PNDs is $e_{1}^{\perp}=\left\langle e_{0}^{a}, e_{2}^{a}, e_{3}^{a}\right\rangle$, where $\left(e_{0}^{a}, e_{i}^{a}\right)$ is the essentially unique Weyl principal tetrad. Furthermore, if we arrange $\left|\lambda_{3}\right| \geq\left|\lambda_{2}\right|$ then type $I\left(M^{+}\right)$is characterized by $\left|\lambda_{3}\right|>\left|\lambda_{2}\right|>\left|\lambda_{1}\right|>0$ and type $I\left(M^{\infty}\right)$ by $\left|\lambda_{3}\right|=\left|\lambda_{2}\right|>\left|\lambda_{1}\right|=0$, and

$$
\left|\lambda_{3}\right|=\left|\lambda_{1}\right|+\left|\lambda_{2}\right|, \quad \sqrt{r_{1}}=\sqrt{r_{2}}+\sqrt{r_{3}}=\left|\lambda_{2}\right|+\left|\lambda_{3}\right|>\sqrt{r_{2}}=\left|\lambda_{3}\right|+\left|\lambda_{1}\right|>\sqrt{r_{3}}=\left|\lambda_{2}\right|-\left|\lambda_{1}\right| .
$$

\section{Principal observer conditions}

Let the Petrov type be I or D at $p$, let $\left(e_{\alpha}^{a}\right) \equiv\left(e_{0}^{a}, e_{i}^{a}\right)$ be a Weyl principal tetrad, and $u^{a}=u^{\alpha} e_{\alpha}^{a}=$ $u^{0} e_{0}^{a}+u^{i} e_{i}^{a}$ an arbitrary observer. Recalling (128) we write $a_{\alpha} \equiv\left(u^{\alpha}\right)^{2}$, implying $a_{0}=1+a_{1}+a_{2}+a_{3}$. We also define

$$
\beta_{i} \equiv \lambda_{j}-\lambda_{k}
$$

and recall $r_{i}=\left|\beta_{i}\right|^{2}$ and $q=\Im\left(\lambda_{j} \bar{\lambda}_{k}\right)$. Note that $\beta_{3}=-\left(\beta_{1}+\beta_{2}\right)$, and $\left(\beta_{1}, \beta_{2}\right) \neq(0,0) \Leftrightarrow\left(r_{1}, r_{2}\right) \neq(0,0)$ by the type I or D assumption. Below we show that the principal observers $\left(\mathcal{P}^{a}=0\right)$ are precisely those with minimal super-energy density $\rho_{\mathrm{g}}=\xi$, and precisely those with $\mathcal{P}^{0}=0$.

C.1 Minimal super-energy density. Consider the expression (121) for the super-energy density:

$$
\rho_{\mathrm{g}}=\xi+\Omega, \quad \Omega=\Phi+12 q A, \quad \Phi=A^{i} r_{i},
$$

where

$$
A^{i}=a_{0} a_{i}-a_{j} a_{k}, \quad A=u^{0} u^{1} u^{2} u^{3} .
$$


In the Petrov type I case (all $r_{i}>0$ ) the unique principal observer is the one with all $u^{i}=0$, while in the Petrov type D case $r_{i}=0 \Leftrightarrow \alpha_{i}=0$ (any fixed $i$ ) the principal observers are those with $u^{j}=u^{k}=0$, and it follows in both cases that $\Omega=0 \Leftrightarrow \rho_{\mathrm{g}}=\xi$ if $u^{a}$ is principal. In [13] Ferrando and Saez proved that $\Omega \geq 0$ as a consequence of $\Phi \geq 0$ and

$$
R \equiv \Phi^{2}-(12 q A)^{2} \geq 0 .
$$

To assert the last inequality they observed that, in view of (117), $R$ can be viewed as a quadratic form in the variables $r_{i}$ :

$$
R=R\left(r_{1}, r_{2}, r_{3}\right)=R^{m n} r_{m} r_{n}, \quad R^{i i}=\left(A^{i}\right)^{2}+4 A^{2}=\left(a_{0} a_{i}+a_{j} a_{k}\right)^{2}, R^{i j}=A^{i} A^{j}-4 A^{2}(i \neq j),
$$

and that $R$ is non-negative since the principal minors of all orders are non-negative:

$$
\begin{aligned}
& \Delta^{i}=R^{i i} \geq 0, \quad \Delta^{i j}=4 A^{2}\left(1+a_{i}+a_{j}\right)^{2}\left(a_{i}+a_{j}\right)^{2} \geq 0, \\
& \Delta^{123}=64 A^{4}\left[\sum_{(i j k)} a_{i}^{2}\left(a_{j}+a_{k}\right)+\sum_{i<j} a_{i} a_{j}+2 a_{1} a_{2} a_{3}\right] \geq 0 .
\end{aligned}
$$

Based on this result applied to Weyl-like tensors and on the Bergqvist-Lankinen theorem 67 the second part of 75D was proved in [39; as affirmed in our main text it follows that $\Omega=0 \Leftrightarrow \rho_{\mathrm{g}}=\xi$ holds only for principal observers. We now give an elementary proof of this fact by simply elaborating the above, where we distinguish between the cases $A \neq 0$ and $A=0$ :

1. $A \neq 0$ (all $u^{i} \neq 0 \Leftrightarrow a_{i}>0$ ). Then $u^{a}$ is not a Weyl principal observer. The quadratic form $R$ is now positive definite since its leading principal minors $\Delta^{1}, \Delta^{12}$ and $\Delta^{123}$ are strictly positive. If $\Omega=0$ then $R=0$, whence all $r_{i}=\left|\beta_{i}\right|^{2}=0$, a contradiction. Hence $\Omega>0$ in this case.

2. $A=0$. Then at least one of the $u^{i}$, s vanishes, say $u^{3}=0 \Leftrightarrow a_{3}=0$. Then $\Omega=A^{i}\left|\beta_{i}\right|^{2}$, and by the triangle inequality we obtain

$$
\begin{aligned}
\Omega & =\left(1+a_{1}+a_{2}\right)\left(a_{1}\left|\beta_{1}\right|^{2}+a_{2}\left|\beta_{2}\right|^{2}\right)-a_{1} a_{2}\left|\beta_{1}+\beta_{2}\right|^{2} \\
& \geq\left(1+a_{1}+a_{2}\right)\left(a_{1}\left|\beta_{1}\right|^{2}+a_{2}\left|\beta_{2}\right|^{2}\right)-a_{1} a_{2}\left[\left|\beta_{1}\right|+\left|\beta_{2}\right|\right]^{2} \\
& =a_{1}\left|\beta_{1}\right|^{2}+a_{2}\left|\beta_{2}\right|^{2}+\left(a_{1}\left|\beta_{1}\right|-a_{2}\left|\beta_{2}\right|\right)^{2} \geq 0 .
\end{aligned}
$$

Hence $\Omega=0 \Leftrightarrow a_{1} \beta_{1}=a_{2} \beta_{2}=0$, and since $\left(\beta_{1}, \beta_{2}\right) \neq(0,0)$ this gives the two qualitatively different cases $\beta_{1}=u^{2}=u^{3}=0$ and $u^{1}=u^{2}=u^{3}=0$. In the former case the Petrov type is $\mathrm{D}$, and in both cases $u^{a}$ is a principal observer. This concludes the proof.

C. $2 \mathcal{P}^{0}=0$. Let $\mathcal{P}^{a}$ be the super-Poynting vector relative to $u^{a}$. By 122 we have

$$
-u^{0} \mathcal{P}^{0}=\Psi+12 q B, \quad \Psi=B^{i} r_{i}
$$

where

$$
B^{i}=a_{0}\left(A^{i}-a_{i} / 2\right)=a_{0}\left[a_{i}\left(a_{0}-1 / 2\right)-a_{j} a_{k}\right], \quad B=\left(a_{0}-1 / 4\right) A .
$$

We now show that $\mathcal{P}^{0}=0$ implies $\mathcal{P}^{a}=0$. In fact, we prove more strongly that 142 holds, which is equivalent to

$$
-u^{0} \mathcal{P}^{0} \geq 0, \quad-u^{0} \mathcal{P}^{0}=0 \Leftrightarrow \mathcal{P}^{a}=0 .
$$

To this end we first show that $\Psi \geq 0$, analogously as in appendix $\mathrm{C}$ of [13]. By (169) one calculates that

$$
\Psi=-Q^{i} p_{i}, \quad Q^{i}=\left(4 a_{0}-2\right) a_{i}+\frac{1}{2}\left(a_{j}+a_{k}\right)+\left(a_{j}-a_{k}\right)^{2} \geq 0 .
$$

By (167)-168) one has $t_{3}=-\left(p_{1}+p_{2}\right)$ and $-p_{3}=\left(q^{2}-p_{1} p_{2}\right) / t_{3} \geq p_{1} p_{2} /\left(p_{1}+p_{2}\right)$, and so

$$
\Psi \geq-Q^{1} p_{1}-Q^{2} p_{2}+Q^{3} \frac{p_{1} p_{2}}{p_{1}+p_{2}}=\frac{Q}{t_{3}}, \quad Q=Q^{1} p_{1}^{2}+Q^{2} p_{2}^{2}+\left(Q^{1}+Q^{2}-Q^{3}\right) p_{1} p_{2} .
$$


The quadratic form $Q$ in the variables $p_{1}$ and $p_{2}$ has principal minors that are non-negative, namely $P^{1}, P^{2} \geq 0$ and determinant $\Delta_{Q}=\frac{9}{4} \sum_{(i j k)} a_{j} a_{k}\left(1+2 a_{j}+a_{k}\right)+9 a_{1} a_{2} a_{3}\left(4 a_{0}-1\right) \geq 0$. Hence $Q \geq 0$ and thus $\Psi \geq 0$, as we wanted to show. Similarly to $(182)$ we can now define $S \equiv \Psi^{2}-(12 q B)^{2}$, which by 168 becomes a quadratic form in the variables $r_{i}$ and has components as in (183) with the symbols $R, A$ replaced by $S, B$. The principal minors of $S$ are calculated to be

$$
\begin{aligned}
& \Delta^{i}=S^{i i}=\left(B^{i}\right)^{2}+4 B^{2} \geq 0, \quad \Delta^{i j}=a_{0}^{2} B^{2}\left(1+2 a_{i}+2 a_{j}\right)^{2}\left(a_{i}+a_{j}\right)^{2} \geq 0, \\
& \Delta^{123}=4 B^{4}\left[6 A+8 A \sum_{i=1}^{3} a_{i}+a_{0} \sum_{(i j k)} a_{j} a_{k}\left(1+a_{j}+a_{k}\right)\left(1+a_{j}+a_{k}\right)\right] \geq 0,
\end{aligned}
$$

such that $S \geq 0$. Hence $\Psi \geq|12 q B|$ and by 185 the first part of 186 is proven. For the second part we argue as before: If $A \neq 0$ then $u^{a}$ is not principal and $\Delta^{1}, \Delta^{12}$ and $\Delta^{123}$ are strictly positive, hence $S>0$ and so $-u^{0} \mathcal{P}^{0}>0$; if $A=0$ with $u^{3}=0 \Leftrightarrow a_{3}=0$ we obtain, analogous to (184):

$$
\begin{aligned}
-\mathcal{P}^{0} / u^{0} & =\left(1 / 2+a_{1}+a_{2}\right)\left(a_{1}\left|\beta_{1}\right|^{2}+a_{2}\left|\beta_{2}\right|^{2}\right)-a_{1} a_{2}\left|\beta_{1}+\beta_{2}\right|^{2} \\
& \geq \frac{1}{2}\left[a_{1}\left|\beta_{1}\right|^{2}+a_{2}\left|\beta_{2}\right|^{2}\right]+\left(a_{1}\left|\beta_{1}\right|-a_{2}\left|\beta_{2}\right|\right)^{2} \geq 0,
\end{aligned}
$$

such that $\mathcal{P}^{0}=0$ is only possible for a principal observer. This concludes the proof.

\section{References}

[1] Bel L Comp. R. Acad. Sci. (Paris) 246, 3015 (1958); 247, 1094 (1958); 2472096 (1958); 248, 1297 (1959); 248, 2561 (1959)

[2] Bel L Ph.D. Thesis C.D.U et S.E.D.E.S Paris 5e

[3] Senovilla J M M Class. Quantum Grav. 17, 2799 (2000)

[4] Matte A Canadian J. Math. 5, 1 (1953)

[5] Landau L D, Lifshitz E M, The classical theory of fields, $4^{\text {th }}$ Ed. (New York: Elsevier, 1975)

[6] García-Parrado Gómez-Lobo A Class. Quant. Grav. 25, 015006 (2008)

[7] Komar A. Phys. Rev. 164, 1595 (1967)

[8] Hawking S W Astrophys. J. 145, 544 (1966)

[9] Teyssandier P, gr-qc/9905080

[10] Garecki J Acta. Phys. Pol. B 8, 159 (1977)

[11] Pirani F Phys. Rev. 105, 1089 (1957)

[12] Bel L Cah. Phys. 16, 59 (1962); English translation: Gen. Rel. Grav. 32, 2047 (2000)

[13] Ferrando J J and Sáez J A Class. Quantum Grav. 29, 075012 (2012)

[14] Fernández-Álvarez F and Senovilla José M. M. Phys. Rev. D 101, 024060 (2020)

[15] Fernández-Álvarez F and Senovilla José M. M., arXiv:2007.11677

[16] Clifton T, Gregoris D and Rosquist K, Class. Quantum Grav. 31, 105012 (2014)

[17] Costa L F and Natário J Gen. Rel. Grav. 46, 1792 (2014) 
[18] Wylleman L Class. Quamtum Grav. 25, 172001 (2008)

[19] Stephani H, Kramer D, MacCallum M A H, Hoenselaers C and Herlt E, Exact Solutions to Einstein's Field Equations (Second Edition) (Cambridge: Cambridge University Press, 2003)

[20] Kinnersley W J. Math. Phys. 10, 1195 (1969)

[21] Plebański J F and Hacyan S J. Math. Phys. 20, 1004 (1979); García D A and Plebański J F J. Math. Phys. 23, 1963 (1982); Debever R, Kamran N and McLenaghan R G Bull. Acad. Roy. Belg. Cl. Sci. 68, 592 (1982); idem J. Math. Phys. 25, 1955 (1984); García D A J. Math. Phys. 25, 1951 (1984)

[22] Synge J L, Relativity: the special theory (North-Holland Publishing Co., Amsterdam, 1956), pp. 334-335

[23] Misner C W and Wheeler J A Ann. Phys. (N.Y.) 2, 525 (1957)

[24] Wheeler J A Phys. Rev. D 16, 3384 (1977)

[25] Misner C W, Thorne K S and Wheeler J A, Gravitation (W. H. Freeman and Co., San Francisco, 1973)

[26] Bini D, Jantzen R T and Miniutti G Int. J. Mod. Phys. D 11, 1439 (2002)

[27] Arianrhod R and McIntosh C B G Class. Quantum Grav. 9, 1969 (1992)

[28] Penrose R and Rindler W, Spinors and Spacetime vol 1 (Cambridge: Cambridge University Press, 1986), pp. $2-4$

[29] Jantzen R T, Carini P and Bini D Ann. Phys. 215, 1 (1992), formatted with corrections on arXiv:gr-qc 0106043

[30] Hall G S, Symmetries and curvature structure in general relativity (Singapore: World Scientific, 2004), p. 174

[31] Ruse H S Proc. London. Math. Soc. 41, 302 (1936)

[32] Coll B Ann. Fond. Louis de Broglie 29, 247 (2004)

[33] Coll B and Ferrando J J J. Math. Phys. 30, 2918 (1989)

[34] V. Bolós, Commun. Math. Phys. 273, 217 (2007)

[35] Hervik S, Ortaggio M and Wylleman L Class. Quantum Grav. 30, 165014 (2013)

[36] Milson R, Coley A, Pravda V and Pravdová A Int. J. Geom. Meth. Mod. Phys. 241 (2005)

[37] Costa L F, Wylleman L and Natário J, arXiv: 1603.03143

[38] Ferrando J J and Sáez J A Gen. Rel. Grav. 41, 1695 (2009)

[39] Ferrando J J and Sáez J A Class. Quantum Grav. 30, 095013 (2013)

[40] Bonilla M A G and Senovilla J M M, Phys. Rev. Lett. 78, 783 (1997)

[41] Debever R, Bull. Soc. Math. Belg. 10112 (1958); idem, Comp. R. Acad. Sci. (Paris) 2491744 (1959); Bel L Sém. méc. analytique méc. céleste 21 (1959); Penrose R Ann. Phys. 10, 171 (1960); Penrose R and Rindler W, Spinors and Spacetime vol 2 (Cambridge: Cambridge University Press, 1986), pp. 224

[42] Ferrando J J, Morales J A and Sáez J A Class. Quantum Grav. 18, 4939 (2001) 
[43] Ferrando J J and Sáez J A Gen. Rel. Grav. 42, 1469 (2010)

[44] Ferrando J J and Sáez J A Gen. Rel. Grav. 36, 2497 (2004)

[45] McIntosh C B G, Arianrhod R, Wade ST and Hoenselaers C Class. Quantum Grav. 11, 1555 (1994)

[46] Stewart J M and Ellis G F R, J. Math. Phys. 9, 1072 (1968)

[47] Goode S W and Wainwright J Gen. Rel. Grav. 18, 315 (1986)

[48] Lozanovski C and Carminati J Class. Quantum Grav. 20, 215 (2003)

[49] Wylleman L and Van den Bergh N Phys. Rev. D 74, 084001 (2006)

[50] Lozanovski C Class. Quantum Grav. 24, 1169 (2007)

[51] Lozanovski C and Wylleman L Class. Quantum Grav. 28, 075015 (2011)

[52] Coley A, Hervik S, Ortaggio M and Wylleman L Class. Quantum Grav. 29, 155016 (2012)

[53] Ferrando J J and Sáez J A Class. Quantum Grav. 14, 129 (1997)

[54] Yang L, Hou X R and Zeng Z, Sci. China Ser. E 36, 628 (1996)

[55] Semerák O Gen. Rel. Grav. 25, 1041 (1993)

[56] Rosquist K Gen. Rel. Grav. 41, 2619 (2009)

[57] Tahvildar-Zadeh A S, arXiv:1410.0416

[58] Feynman R, Leighton R, Sands M, The Feynman Lectures on Physics Vol. II (Addison-Wesley Publishing Company, 1964), Secs. 27.5-27.6.

[59] Bonnor W B, Phys. Lett. A 158, 23 (1991).

[60] Herrera L, González G A, Pachón L A, Rueda J. A., Class. Quantum Grav. 23, 2395 (2006)

[61] Herrera L, Barreto W, Phys. Rev. D 86, 064014 (2012)

[62] Costa L F, Franco R, Cardoso V, Phys. Rev. D 98, 024026 (2018)

[63] Herrera L, Carrot J, Di Prisco A, Phys. Rev. D 76044012 (2007)

[64] Herrera L, Ibáñez J, Di Prisco A, Phys. Rev. D 87, 087503 (2013)

[65] Penrose R and Rindler W, Spinors and Spacetime vol 2 (Cambridge: Cambridge University Press, 1986)

[66] McIntosh C B G and Arianrhod R Class. Quantum Grav. 7, L213 (1990)

[67] Bergqvist G and Lankinen P Class. Quantum Grav. 21, 3499 (2004) 